\title{
The Fekete-Szegö theorem with splitting conditions: Part II
}

by

\author{
Robert Rumely (Athens, GA)
}

1. Introduction. A classical theorem of Fekete and Szegö ([5]) says that if $E \subseteq \mathbb{C}$ is a compact set with logarithmic capacity $\gamma(E) \geq 1$, stable under complex conjugation, then every complex neighborhood of $E$ contains infinitely many conjugate sets of algebraic integers. Raphael Robinson [10] strengthened this, showing that if $E \subseteq \mathbb{R}$, then every real neighborhood of $E$ contains infinitely many conjugate sets of totally real algebraic integers.

In [3], Cantor stated a theorem of Fekete-Szegö-Robinson type for adelic sets in $\mathbb{P}^{1}$ over a number field $K$. Write $K_{v}$ for the completion of $K$ at a place $v$. Fix algebraic closures $\widetilde{K}$ of $K, \widetilde{K}_{v}$ of $K_{v}$, and let $\mathbb{C}_{v}$ be the completion of $\widetilde{K}_{v}$. If $v$ is nonarchimedean, let $\widehat{O}_{v}$ be the ring of integers of $\mathbb{C}_{v}$. For each $v$, let a Galois-stable set $E_{v} \subset \mathbb{P}^{1}\left(\mathbb{C}_{v}\right)$ and a neighborhood $U_{v}$ of $E_{v}$ in $\mathbb{P}^{1}\left(\mathbb{C}_{v}\right)$ be given. Cantor developed a theory of capacity for adelic sets $\mathbb{E}=\prod_{v} E_{v} \subset \prod_{v} \mathbb{P}^{1}\left(\mathbb{C}_{v}\right)$, and defined the capacity $\gamma(\mathbb{E}, \mathfrak{X})$ for such a set with respect to a finite set of global points $\mathfrak{X} \subset \mathbb{P}^{1}(\widetilde{K})$. Let $S$ be a finite set of places of $K$ such that for each $v \in S$, there is a finite Galois extension $L_{w} / K_{v}$ with $E_{v} \subset \mathbb{P}^{1}\left(L_{w}\right)$. Cantor's "FeketeSzegő theorem with splitting conditions" ([3, Theorem 5.1.1, p. 199]) asserts that if $\gamma(\mathbb{E}, \mathfrak{X}) \geq 1$, then there are infinitely many points in $\mathbb{P}^{1}(\widetilde{K})$ whose conjugates in $\mathbb{C}_{v}$ belong to $U_{v}$ for all $v$, and lie in $\mathbb{P}^{1}\left(L_{w}\right)$, if $v \in S$. We call the latter constraints "splitting conditions". Unfortunately, there were gaps in Cantor's proof. An explicit error occurs in his Lemma 4.2.6, where an adelic polynomial is asserted to have coefficients in a number field. There are also significant difficulties in both the archimedean and nonarchimedean patching processes which his construction did not come to terms with.

2000 Mathematics Subject Classification: Primary 11R06, 12D10, 31C15; Secondary 11R04, 14G25, 14G40.

Key words and phrases: Fekete-Szegő theorem, capacity theory, splitting conditions.

Work supported in part by NSF grants DMS 9500842 and DMS 0070736. 
Recently the author [12] established the theorem in the special case where $\mathfrak{X}=\{\infty\}$ and $K=\mathbb{Q}$, with $E_{\infty}=[-2 r, 2 r]$, and $E_{p}=\mathbb{Z}_{p}$ for finitely many primes $p$. In this paper, we prove the theorem for $\mathfrak{X}=\{\infty\}$ over an arbitrary number field $K$, and for general sets $E_{v}$. We also strengthen the result, showing that under appropriate hypotheses on the $E_{v}$, the algebraic numbers produced exist in all sufficiently large degrees.

The author would like to thank Peter Rice for pointing out the lemma on the "Alabama Paradox" used in Section 8.

2. Statement of the results. Let $K$ be a number field. For each place $v$ of $K$, let a set $E_{v} \subset \mathbb{C}_{v}$, and a $\mathbb{C}_{v}$-neighborhood $U_{v}$ of $E_{v}$, be given. Assume that $E_{v}$ is bounded and stable under the group of continuous automorphisms $\operatorname{Gal}_{\mathrm{c}}\left(\mathbb{C}_{v} / K_{v}\right)$ for each $v$, and that $E_{v}=U_{v}=\widehat{O}_{v}$ for all but finitely many $v$. Write $\log (x)=\ln (x)$. For a compact set $E_{v}$, the local capacity $\gamma\left(E_{v}\right)$ can be defined by

$$
\gamma\left(E_{v}\right)=e^{-V} \quad \text { with } \quad V=\inf _{\nu} \iint_{E_{v} \times E_{v}}-\log \left(|z-w|_{v}\right) d \nu(z) d \nu(w),
$$

where the inf is taken over all positive measures $\nu$ of total mass 1 supported on $E_{v}$. There are several other formulas for the local capacity; these, and methods of computing local capacities for certain classes of sets, will be recalled below. For an arbitrary set $E_{v}$, the local capacity is given by

$$
\gamma\left(E_{v}\right)=\sup _{\substack{F_{v} \subset E_{v} \\ F_{v} \text { compact }}} \gamma\left(F_{v}\right) .
$$

Our $\gamma\left(E_{v}\right)$ is sometimes called the "inner capacity" (see for example [11], [13]). For archimedean compact $E_{v}$, the local capacity $\gamma\left(E_{v}\right)$ is just the classical logarithmic capacity, or "transfinite diameter". For nonarchimedean compact $E_{v}$, it is the $v$-adic generalization of the transfinite diameter, first studied by Bertrandias (see [1]).

Cantor's theory defines the capacity $\gamma(\mathbb{E}, \mathfrak{X})$ of an adelic set $\mathbb{E}=\prod_{v} E_{v}$ with respect to a finite set of global algebraic points $\mathfrak{X}$. When $\mathfrak{X}=\{\infty\}$, Cantor's capacity reduces to a weighted product of local capacities:

$$
\gamma(\mathbb{E}):=\gamma(\mathbb{E},\{\infty\})=\prod_{v} \gamma\left(E_{v}\right)^{D_{v}}
$$

The weights $D_{v}$ are the same as the ones in the product formula for $K$ : they (and the local capacities) depend on the normalization of the absolute values $|x|_{v}$.

In this paper we normalize absolute values as follows. If $v$ is archimedean, we take $|x|_{v}=|x|$ to be the usual absolute value on $\mathbb{R}$ or $\mathbb{C}$. From an arithmetic point of view it would be preferable to take $|x|_{v}=|x|^{2}$ when $K_{v} \cong$ $\mathbb{C}$, but we use the normalization above to avoid confusion concerning the 
literature about capacities of sets in $\mathbb{C}$. If $v$ is nonarchimedean, we take $|x|_{v}$ to be the canonical absolute value on $K_{v}$ given by the modulus of additive Haar measure (if $\pi_{v}$ is a uniformizing element at $v$, then $\left|\pi_{v}\right|_{v}=1 / q_{v}$, where $q_{v}$ is the order of the residue field of $K_{v}$ ). With this normalization, $D_{v}=1$ unless $v$ is archimedean and $K_{v} \cong \mathbb{C}$, in which case $D_{v}=2$.

For nonarchimedean $v$, let $\log _{v}(x)$ denote the logarithm to the base $q_{v}=$ $\#\left(O_{v} / \pi_{v} O_{v}\right)$. For archimedean $v$, write $\log _{v}(x)=\log (x)=\ln (x)$.

For each $v$, the absolute value $|x|_{v}$ on $K_{v}$ extends uniquely to absolute values on $\widetilde{K}_{v}$ and $\mathbb{C}_{v}$, and we denote these extensions by $|x|_{v}$ as well. The Galois group $\operatorname{Gal}\left(\widetilde{K}_{v} / K_{v}\right)$ respects $|x|_{v}$, and its action on $\widetilde{K}_{v}$ extends to a continuous action on $\mathbb{C}_{v}$. Conversely, each continuous automorphism of $\mathbb{C}_{v} / K_{v}$ arises from an element of $\operatorname{Gal}\left(\widetilde{K}_{v} / K_{v}\right)$. By $\operatorname{Gal}_{\mathrm{c}}\left(\mathbb{C}_{v} / K_{v}\right) \cong \operatorname{Gal}\left(\widetilde{K}_{v} / K_{v}\right)$ we mean the group of continuous automorphisms of $\mathbb{C}_{v}$, fixing $K_{v}$. Given $a_{v} \in \mathbb{C}_{v}$, and $r_{v}>0$, let the "open" and "closed" balls be

$$
\begin{aligned}
B\left(a_{v}, r_{v}\right)^{-} & =\left\{z \in \mathbb{C}_{v}:\left|z-a_{v}\right|_{v}<r_{v}\right\}, \\
B\left(a_{v}, r_{v}\right) & =\left\{z \in \mathbb{C}_{v}:\left|z-a_{v}\right|_{v} \leq r_{v}\right\} .
\end{aligned}
$$

By an adelic neighborhood $\mathbb{U}$ of $\mathbb{E}$, we mean a set $\mathbb{U}=\prod_{v} U_{v}$, where each $U_{v}$ is an open set in $\mathbb{C}_{v}$ containing $E_{v}$.

Our main theorem is as follows:

TheOREM 2.1 (Fekete-Szegő theorem with splitting conditions). Let $K$ be a number field, and let $\mathbb{E}=\prod_{v} E_{v}$ be an adelic set over $K$ such that each $E_{v}$ is bounded and stable under $\mathrm{Gal}_{\mathrm{c}}\left(\mathbb{C}_{v} / K_{v}\right)$, with $E_{v}=\widehat{O}_{v}$ for all but finitely many $v$. Suppose that $\prod_{v} \gamma\left(E_{v}\right)^{D_{v}}>1$, or that $\prod_{v} \gamma\left(E_{v}\right)^{D_{v}}=1$ and $E_{v}$ is compact for at least one archimedean $v$. Let $S$ be a finite set of places of $K$, such that for each $v \in S$ there is a finite Galois extension $L_{w} / K_{v}$ with $E_{v} \subset L_{w}$. Then for each adelic neighborhood $\mathbb{U}=\prod_{v} U_{v}$ of $\mathbb{E}$, there are infinitely many numbers $\alpha \in \widetilde{K}$ with the following properties:

(1) for each $v$, all the conjugates of $\alpha$ in $\mathbb{C}_{v}$ belong to $U_{v}$;

(2) for each $v \in S$, these conjugates belong to $L_{w} \cap U_{v}$.

Under appropriate hypotheses, we can require that the numbers $\alpha$ produced by Theorem 2.1 belong to the sets $E_{v}$ :

TheOREM 2.2. Let $K$ be a number field, and let $\mathbb{E}=\prod_{v} E_{v}$ be an adelic set over $K$ such that each $E_{v}$ is bounded and stable under $\mathrm{Gal}_{\mathrm{c}}\left(\mathbb{C}_{v} / K_{v}\right)$, and $E_{v}=\widehat{O}_{v}$ for all but finitely many $v$. Assume that $\prod_{v} \gamma\left(E_{v}\right)^{D_{v}}>1$ and that

(1) for each archimedean $v$, either

(A) $E_{v}$ is the closure of its interior, and $\partial E_{v}$ is a finite union of arcs; or

(B) $K_{v} \cong \mathbb{R}$, and $E_{v} \subset \mathbb{R}$ is a finite union of closed intervals; 
(2) for each nonarchimedean $v$, either

(A) $E_{v}$ is a finite union of open and/or closed balls in $\mathbb{C}_{v}$; or

(B) $E_{v}=\bigcup_{i=1}^{M}\left(a_{i}+\pi_{w}^{k_{i}} O_{w}\right) \subset L_{w}$ is a finite union of cosets of the ring of integers of a finite Galois extension $L_{w} / K_{v}$.

Then there are infinitely many $\alpha \in \widetilde{K}$ such that for each $v$, all the conjugates of $\alpha$ in $\mathbb{C}_{v}$ belong to $E_{v}$.

Theorem 2.2 follows from Theorem 2.1 by shrinking the archimedean $E_{v}$ slightly, and taking the $U_{v}$ to be the interiors of the original sets.

In a different direction, we can ask whether for sufficiently large $n$, numbers $\alpha$ as in Theorems 2.1 and 2.2 can be found with $[K(\alpha): K]=n$. In general this is not possible; for example if $K=\mathbb{Q}$ and the archimedean set $E_{\infty}$ and neighborhood $U_{\infty}$ are stable under complex conjugation but do not meet $\mathbb{R}$, then the numbers $\alpha$ produced must be totally complex, so $[\mathbb{Q}(\alpha): \mathbb{Q}]$ must be even. Similar constraints arise at finite places. However, we can prove:

TheOREM 2.3. Let $K$ be a number field, and let $\mathbb{E}$ and $\mathbb{U}$ satisfy the hypotheses of Theorem 2.1 (resp. Theorem 2.2). Suppose in addition that each $E_{v}$ contains a point of $K_{v}$, and that $L_{w}=K_{v}$ for each $v \in S$. Then for all sufficiently large $n$, there exist algebraic numbers $\alpha$ satisfying the conclusions of Theorem 2.1 (resp. Theorem 2.2) with $[K(\alpha): K]=n$. In addition, if $S_{1}^{\prime}$ and $S_{2}^{\prime}$ are finite sets of nonarchimedean places of $K$ where $E_{v}=\widehat{O}_{v}$, disjoint from $S$ and from each other, then for each $v \in S_{1}^{\prime}$ we can require that $v$ is inert in $K(\alpha) / K$, and for each $v \in S_{2}^{\prime}$ we can require that $v$ is totally ramified.

It follows from Theorem 2.3 that there is a number $Q$, depending only on $\mathbb{E}$ and $\mathbb{U}$, such that the numbers $\alpha$ in Theorems 2.1 and 2.2 can be found with $[K(\alpha): K]=n$ for all sufficiently large $n$ divisible by $Q$.

The following concrete example generalizes the main theorem of [12]:

Corollary 2.4. Let $\mathbb{E}=\prod_{v} E_{v}$ be an adelic set over $K$ such that

(1) for each archimedean $v$ with $K_{v} \cong \mathbb{C}, E_{v}=B\left(a_{v}, r_{v}\right)$ is a closed ball;

(2) for each archimedean $v$ with $K_{v} \cong \mathbb{R}, E_{v}=\left[a_{v}, b_{v}\right]$ is a closed interval;

(3) for each nonarchimedean $v$ in a finite set of places $S, E_{v}=O_{v}$, the ring of integers of $K_{v}$;

(4) for the remaining nonarchimedean $v, E_{v}=\widehat{O}_{v}$.

Assume

$$
\prod_{K_{v} \cong \mathbb{C}} r_{v}^{2} \cdot \prod_{K_{v} \cong \mathbb{R}} \frac{b_{v}-a_{v}}{4} \cdot \prod_{v \in S} q_{v}^{-1 /\left(q_{v}-1\right)}>1 .
$$

Then for all sufficiently large $n$, there exist numbers $\alpha$ with $[K(\alpha): K]=n$ whose conjugates all belong to $E_{v}$, for each $v$. 
Algebraic numbers satisfying a variety of congruence conditions, with controlled archimedean conjugates, can be constructed by imposing appropriate geometric conditions on the sets $E_{v}$. As a whimsical example, taking $K=\mathbb{Q}$ in Theorem 2.2, with $E_{\infty}=B(0,2+\varepsilon), E_{2}=\mathbb{Z}_{2}, E_{3}=\mathbb{Z}_{3}^{\text {nr }}$ (the ring of integers of the maximal unramified extension of $\left.\mathbb{Q}_{3}\right), E_{5}=B_{5}(1,1)^{-}$, $E_{7}=B_{7}(0,1)^{-}$, and $E_{p}=\widehat{O}_{p}$ for all remaining $p$, we have

COROLlary 2.5. For any $\varepsilon>0$, there are infinitely many algebraic integers $\alpha$ such that

(1) the archimedean conjugates of $\alpha$ satisfy $|\sigma(\alpha)|<2+\varepsilon$;

(2) the prime 2 splits completely in $\mathbb{Q}(\alpha)$;

(3) the prime 3 is unramified in $\mathbb{Q}(\alpha)$;

(4) at all places $v$ of $\mathbb{Q}(\alpha)$ above 5 , we have $\alpha \equiv 1\left(\bmod \wp_{v}\right)$;

(5) at all places $v$ of $\mathbb{Q}(\alpha)$ above 7 , we have $\alpha \in \wp_{v}$.

Here, $\gamma(B(0,2+\varepsilon))=2+\varepsilon$, and $\gamma\left(\mathbb{Z}_{2}\right)=2^{-1 /(2-1)}=1 / 2$. The condition "3 is unramified" is cost-free, since

$$
\gamma\left(\mathbb{Z}_{3}^{\mathrm{nr}}\right)=\sup _{\substack{L_{w} / \mathbb{Q}_{3} \\ \text { finite, unramified }}} \gamma\left(O_{w}\right)=\lim _{m \rightarrow \infty} 3^{-1 /\left(3^{m}-1\right)}=1 .
$$

Likewise, the "open" conditions at the primes 5 and 7 do not impose any cost, since the capacities of the sets $B_{5}(1,1)^{-}$and $B_{7}(0,1)^{-}$are both 1 : the capacity of an open or closed ball is simply its radius, $r$. For all other primes, $\gamma\left(\widehat{O}_{p}\right)=1$. Thus, $\gamma(\mathbb{E})=1+\varepsilon / 2>1$.

We should also mention a converse to the Fekete-Szegö theorem, which shows that the hypothesis $\gamma(\mathbb{E}) \geq 1$ is sharp. By a $P L$-domain in $\mathbb{C}_{v}$ ("Polynomial Lemniscate Domain") we mean a set of the form

$$
U_{v}=\left\{z \in \mathbb{C}_{v}:\left|f_{v}(x)\right|_{v} \leq R_{v}\right\}
$$

where $f_{v}(z) \in \mathbb{C}_{v}[z]$ is a nonconstant polynomial, and $R_{v}$ belongs to the value group of $\mathbb{C}_{v}^{\times}$. For a set $E_{v} \subset \mathbb{C}_{v}$, we say that $E_{v}$ is algebraically capacitable if

$$
\gamma\left(E_{v}\right)=\inf _{\substack{U_{v} \supset E_{v} \\ U_{v}=\text { PL-domain }}} \gamma\left(U_{v}\right) .
$$

For archimedean $v$, Hilbert's Lemniscate Theorem shows that compact sets are algebraically capacitable (see [11, Proposition 3.3.3]). For nonarchimedean $v$, it is shown in $[11, \S 4.4]$ that compact sets, PL-domains, and any finite combination of unions or intersections of such sets, are algebraically capacitable; in addition, finite unions of open balls or closed balls are algebraically capacitable. The following is a special case of [3, Theorem 5.1.2, p. 199]; see [11, Theorem 6.3.1] for a generalization to curves: 
Theorem 2.6 (Fekete's theorem). Let $\mathbb{E}=\prod_{v} E_{v}$ be an affine adelic set over $K$ such that each $E_{v}$ is bounded, stable under $\mathrm{Gal}_{\mathrm{c}}\left(\mathbb{C}_{v} / K_{v}\right)$, and algebraically capacitable, such that

$$
\prod_{v} \gamma\left(E_{v}\right)^{D_{v}}<1
$$

Then there exists an adelic neighborhood $\mathbb{U}=\prod_{v} U_{v}$ of $\mathbb{E}$, which contains only finitely many algebraic numbers $\alpha$ whose conjugates in $\mathbb{C}_{v}$ belong to $U_{v}$ for all $v$.

3. Local capacities. In this section we will recall some of the properties of the capacity, and explain how to compute local capacities for certain types of sets.

3.1. The archimedean case. If $v$ is archimedean, fix an isomorphism of $\widetilde{K}_{v}$ with $\mathbb{C}$, and regard $E_{v}$ as a subset of $\mathbb{C}$. Assume $E_{v}$ is compact; then $\gamma\left(E_{v}\right)$ is the classical logarithmic capacity. Three well-known formulas for the logarithmic capacity are as follows (see for example [13, pp. 71-75]):

$$
\begin{aligned}
\gamma\left(E_{v}\right) & =\lim _{n \rightarrow \infty} \sup _{\left\{x_{1}, \ldots, x_{n}\right\} \subseteq E_{v}}\left(\prod_{i \neq j}\left|x_{i}-x_{j}\right|\right)^{1 /\left(n^{2}-n\right)} \\
& =\lim _{n \rightarrow \infty} \inf _{\substack{f(x) \in \mathbb{C}[x] \\
\text { monic, } \operatorname{deg}(f)=n}}\left(\|f(x)\|_{E_{v}}\right)^{1 / n} \\
& =e^{-V\left(E_{v}\right)}
\end{aligned}
$$

where $\|f(x)\|_{E_{v}}$ is the sup norm of $f(x)$ on $E_{v}$ under $|x|_{v}$, and

$$
V\left(E_{v}\right)=\inf _{\nu} \iint_{E_{v} \times E_{v}}-\log (|z-w|) d \nu(z) d \nu(w)
$$

where $\nu$ runs over probability measures (positive measures of total mass 1 ) supported on $E_{v}$.

The first formula, $\gamma\left(E_{v}\right)$ as the "transfinite diameter", was the definition originally used by Fekete. It shows that $\gamma\left(E_{v}\right)$ is translation-invariant and homogeneous under scaling. The second formula, $\gamma\left(E_{v}\right)$ as the "Chebyshev constant", provides a connection with polynomials. Let $\partial E_{v}^{\text {out }}$ be the outer boundary of $E_{v}$, the part of $\partial E_{v}$ meeting the closure of the unbounded component of $\mathbb{C} \backslash E_{v}$. By the maximum principle, $\gamma\left(E_{v}\right)=\gamma\left(\partial E_{v}\right)=\gamma\left(\partial E_{v}^{\text {out }}\right)$.

In the third formula, the number $V\left(E_{v}\right)$ is called the "Robin constant" of $E_{v}$. If $\gamma\left(E_{v}\right)>0$ (equivalently, if $V\left(E_{v}\right)<\infty$ ), there is a unique probability measure $\mu$, called the equilibrium distribution of $E_{v}$, for which the inf is achieved. This holds, for example, if $E_{v}$ contains an arc. When $\gamma\left(E_{v}\right)>0$, we define the "Green function of $E_{v}$ relative to the point $\infty$ " by 


$$
G\left(z, \infty ; E_{v}\right)=V\left(E_{v}\right)+\int_{E_{v}} \log (|z-w|) d \mu(w) .
$$

The Green function is characterized by the following properties: it is continuous in the complement of $E_{v}$ and upper semicontinuous everywhere; it is 0 on $E_{v}$ except possibly on a set of capacity 0 contained in $\partial E_{v}^{\text {out }}$, and it is harmonic in $\mathbb{C} \backslash E_{v}$ with a logarithmic pole at $\infty$ (that is, $G\left(z, \infty ; E_{v}\right)-\log (|z|)$ remains bounded as $z \rightarrow \infty)$. The restriction of $G\left(z, \infty ; E_{v}\right)$ to the unbounded component of $\mathbb{C} \backslash E_{v}$ is the classical Green function $g(z, \infty)$ of that domain. Clearly

$$
V\left(E_{v}\right)=\lim _{z \rightarrow \infty}\left(G\left(z, \infty ; E_{v}\right)-\log (|z|)\right) .
$$

If $\partial E_{v}$ is a union of arcs, then $G\left(z, \infty ; E_{v}\right)$ is continuous everywhere, and $G\left(z, \infty ; E_{v}\right)=0$ on $E_{v}$ : the exceptional set in $\partial E_{v}$ is empty.

Formula (3.1) and the characterization of the Green function make it possible to compute the capacities of many sets: if $G\left(z, \infty ; E_{v}\right)$ can be found, then the capacity can be read off. In particular, for a closed ball $B(a, r)$,

$$
\begin{aligned}
G(z, \infty ; B(a, r)) & = \begin{cases}\log (|(z-a) / r|) & \text { if } z \notin B(a, r), \\
0 & \text { if } z \in B(a, r),\end{cases} \\
\gamma(B(a, r)) & =r .
\end{aligned}
$$

If an explicit conformal mapping $f(z)$ can be found which takes $\mathbb{C} \backslash E_{v}$ to $\mathbb{C} \backslash B(0,1)$, then $G\left(z, \infty ; E_{v}\right)=\log (|f(z)|)$ for $z \notin E_{v}$. In this way, capacities can be computed for straight line segments, ellipses, triangles, rectangles, regular $n$-gons, bent segments, arcs of circles, and so on; a fairly extensive table of capacities for archimedean sets is given in [11, pp. 348-351]. For a segment,

$$
\gamma([a, b])=(b-a) / 4 \text {. }
$$

For an ellipse $E_{v}=\left\{x+i y \in \mathbb{C}: x^{2} / a^{2}+y^{2} / b^{2} \leq 1\right\}$,

$$
\gamma\left(E_{v}\right)=(a+b) / 2 \text {. }
$$

More generally, if $h(z)$ is a monic polynomial of degree $n$, and $F_{v}=$ $h^{-1}\left(E_{v}\right)$, then $G\left(z, \infty ; F_{v}\right)=(1 / n) G\left(h(z), \infty ; E_{v}\right)$, giving the pullback formula

$$
\gamma\left(h^{-1}\left(E_{v}\right)\right)=\gamma\left(E_{v}\right)^{1 / n}
$$

Two consequences of the pullback formula we will need are:

Corollary 3.1. Let $h(z) \in \mathbb{C}[z]$ be monic of degree $n$.

(A) If $E_{v}=\left\{z \in \mathbb{C}:|h(z)| \leq R_{v}^{n}\right\} \subset \mathbb{C}$, then $\gamma\left(E_{v}\right)=R_{v}$.

(B) If $E_{v}=\bigcup_{i=1}^{M}\left[a_{i}, b_{i}\right] \subset \mathbb{R}$ is a finite union of segments, and if $h(z) \in$ $\mathbb{R}[z]$ "oscillates $n$ times between $\pm 2 R_{v}^{n}$ on $E_{v}$ " (so that $E_{v}=\{z \in \mathbb{C}$ : $\left.\left.|h(z)| \leq 2 R_{v}^{n}\right\}\right)$, then $\gamma\left(E_{v}\right)=R_{v}$. 
3.2. The nonarchimedean case: $P L$-domains. If $K_{v}$ is nonarchimedean let $h(z) \in \mathbb{C}_{v}[z]$ be a monic polynomial of degree $n$, and let $R_{v}$ belong to the value group of $\mathbb{C}_{v}^{\times}$. Following Cantor, we call a set of the form

$$
E_{v}=\left\{z \in \mathbb{C}_{v}:|h(z)|_{v} \leq R_{v}^{n}\right\}
$$

a Polynomial Lemniscate Domain ("PL-domain"). Motivated by the pullback formula in the archimedean case, Cantor ([3, §3.2]) defined the capacity, Robin constant, and Green function of $E_{v}$ by

$$
\begin{aligned}
\gamma\left(E_{v}\right) & =R_{v}, \\
V\left(E_{v}\right) & =-\log _{v}\left(R_{v}\right), \\
G\left(z, \infty ; E_{v}\right) & = \begin{cases}(1 / n) \log _{v}\left(|h(z)|_{v} / R_{v}^{n}\right) & \text { if } z \notin E_{v}, \\
0 & \text { if } z \in E_{v},\end{cases}
\end{aligned}
$$

and showed that these are independent of the choice of $h(z)$.

Note that if $E_{v}=\widehat{O}_{v}$, then taking $f(z)=z$ and $R_{v}=1$ in the formulas above yields $\gamma\left(\widehat{O}_{v}\right)=1$. In our definition of an adelic set $\mathbb{E}$, we required that $E_{v}=\widehat{O}_{v}$ for all but finitely many $v$. Thus, the global capacity

$$
\gamma(\mathbb{E})=\prod_{v} \gamma\left(E_{v}\right)^{D_{v}}
$$

is in fact a finite product.

An important part of Cantor's theory was the identification of PLdomains with "finite unions of balls", sets of the form

$$
E_{v}=\bigcup_{i=1}^{M} B\left(a_{i}, r_{i}\right),
$$

where each $B\left(a_{i}, r_{i}\right)=\left\{z \in \mathbb{C}_{v}:\left|z-a_{i}\right|_{v} \leq r_{i}\right\}$ is a closed ball, with radius $r_{i}$ in the value group of $\mathbb{C}_{v}^{\times}$. By the ultrametric inequality, we can assume the union is disjoint, which means that $\left|a_{i}-a_{j}\right|_{v}>\max \left(r_{i}, r_{j}\right)$ for each $i \neq j$. Cantor showed, by induction on the number of zeros of $h(z)$, that each PL-domain was a finite union of balls ([3, Theorem 3.12, p. 180]).

Conversely, given a finite union of balls as above, he showed that there was a polynomial realizing it as a PL-domain. The construction is as follows. Define the $(M+1) \times(M+1)$ symmetric real matrix

$$
\Theta_{v}=\left[\begin{array}{ccccc}
0 & 1 & 1 & \cdots & 1 \\
1 & \log _{v}\left(r_{1}\right) & \log _{v}\left(\left|a_{1}-a_{2}\right|_{v}\right) & \cdots & \log _{v}\left(\left|a_{1}-a_{M}\right|_{v}\right) \\
1 & \log _{v}\left(\left|a_{2}-a_{1}\right|_{v}\right) & \log _{v}\left(r_{2}\right) & \cdots & \log _{v}\left(\left|a_{2}-a_{M}\right|_{v}\right) \\
\vdots & \vdots & \vdots & \ddots & \vdots \\
1 & \log _{v}\left(\left|a_{M}-a_{1}\right|_{v}\right) & \log _{v}\left(\left|a_{M}-a_{2}\right|_{v}\right) & \cdots & \log _{v}\left(r_{M}\right)
\end{array}\right] .
$$


Then $\Theta_{v}$ is nonsingular, and, in the unique solution to the system of equations

$$
\left[\begin{array}{c}
1 \\
0 \\
\vdots \\
0
\end{array}\right]=\Theta_{v}\left[\begin{array}{c}
V\left(E_{v}\right) \\
w_{1} \\
\vdots \\
w_{M}
\end{array}\right],
$$

the numbers $w_{i}$ are positive. (For a proof, see Appendix II below.) Since $\Theta_{v}$ has rational entries, $V\left(E_{v}\right)$ and the $w_{i}$ are rational as well. Let $n$ be a common denominator for the $w_{i}$. By Lemma 12.1 of Appendix II, a polynomial realizing $E_{v}$ as a PL-domain is given by

$$
h(z)=\prod_{i=1}^{M}\left(z-a_{i}\right)^{n w_{i}},
$$

with $R_{v}^{n}=q_{v}^{-n V\left(E_{v}\right)}$. It follows that

$$
\gamma\left(E_{v}\right)=q_{v}^{-V\left(E_{v}\right)}
$$

Thus, the problem of computing the capacity for finite unions of balls reduces to linear algebra.

3.3. The nonarchimedean case: compact sets. If $v$ is nonarchimedean and $E_{v}$ is compact, we define the Robin constant

$$
V\left(E_{v}\right)=\inf _{\nu} \iint_{E_{v} \times E_{v}}-\log _{v}\left(|z-w|_{v}\right) d \nu(z) d \nu(w)
$$

where the inf is taken over all probability measures $\nu$ supported on $E_{v}$. If $V\left(E_{v}\right)$ is finite, there is a unique probability measure $\mu$, the equilibrium distribution, for which the inf is achieved (see [11, §4.1]). By analogy with the archimedean case, we define the capacity and Green function of $E_{v}$ by

$$
\begin{aligned}
\gamma\left(E_{v}\right) & =q_{v}^{-V\left(E_{v}\right)}, \\
G\left(z, \infty ; E_{v}\right) & =V\left(E_{v}\right)+\int_{E_{v}} \log _{v}\left(|z-w|_{v}\right) d \mu(w) .
\end{aligned}
$$

It is often convenient to work with the potential function

$$
\omega_{\mu}\left(z ; E_{v}\right)=\int_{E_{v}}-\log _{v}\left(|z-w|_{v}\right) d \mu(w)=V\left(E_{v}\right)-G\left(z, \infty ; E_{v}\right)
$$

rather than the Green function. The equilibrium distribution $\mu$ is characterized by the property that $\omega_{\mu}\left(z ; E_{v}\right)$ takes a constant value a.e. on $E_{v}$ (here a.e. means except on a set of capacity 0); clearly this value is $V\left(E_{v}\right)$.

For example, take $E_{v}=a+b O_{v}$. Since $E_{v}$ is translation invariant under $b O_{v}$, the equilibrium distribution $\mu$ is translation invariant as well. Thus 
$\mu$ must be additive Haar measure restricted to $E_{v}$, normalized so that $\mu\left(E_{v}\right)=1$. Put $r=|b|_{v}$. It is not hard to compute that

$$
\omega_{\mu}\left(z ; E_{v}\right)= \begin{cases}\frac{1}{q_{v}-1}-\log _{v}(r) & \text { for } z \in E_{v}, \\ -\log _{v}\left(|z-a|_{v}\right) & \text { for }|z-a|_{v}>r,\end{cases}
$$

so that $\gamma\left(E_{v}\right)=r \cdot q_{v}^{-1 /\left(q_{v}-1\right)}$. In particular, when $K_{v}=\mathbb{Q}_{p}$ and $E_{v}=\mathbb{Z}_{p}$,

$$
\gamma\left(\mathbb{Z}_{p}\right)=p^{-1 /(p-1)},
$$

a formula used implicitly in [12].

More generally, suppose $E_{v} \subset K_{v}$ is a disjoint union of cosets

$$
\bigcup_{i=1}^{M}\left(a_{i}+b_{i} O_{v}\right)
$$

Put $r_{i}=\left|b_{i}\right|_{v}$ for each $i$; the disjointness means that $\max \left(r_{i}, r_{j}\right)>\left|a_{i}-a_{j}\right|_{v}$ for all $i \neq j$. Using the ultrametric inequality and the characterization of $\mu$, one sees that $\mu$ must be a weighted sum of the equilibrium distributions $\mu_{i}$ of the cosets $E_{v, i}=a_{i}+b_{i} O_{v}$ :

$$
\mu=\sum_{i=1}^{M} w_{i} \mu_{i}
$$

We claim that $V\left(E_{v}\right)$ and the $w_{i}$ satisfy a system of equations much like the ones that determine the polynomial $h(z)$ for a PL-domain. Indeed, let $\Theta_{v}$ be defined as in (3.5) for $\bigcup_{i=1}^{M} B\left(a_{i}, r_{i}\right)$, and $I_{0}$ be the $(M+1) \times(M+1)$ matrix

$$
I_{0}=\left[\begin{array}{ccccc}
0 & 0 & 0 & \cdots & 0 \\
0 & 1 & 0 & \cdots & 0 \\
0 & 0 & 1 & \cdots & 0 \\
\vdots & \vdots & \vdots & \ddots & 0 \\
0 & 0 & 0 & \cdots & 1
\end{array}\right]
$$

The fact that $\omega_{\mu}\left(z ; E_{v}\right)$ must take the same value on each $E_{v, i}$ yields the system of equations

$$
\left[\begin{array}{l}
1 \\
\overrightarrow{0}
\end{array}\right]=\left(\Theta_{v}-\frac{1}{q_{v}-1} I_{0}\right)\left[\begin{array}{c}
V\left(E_{v}\right) \\
\vec{w}
\end{array}\right] .
$$

More explicitly, since $-\log _{v}\left(\left|a_{i}-a_{j}\right|_{v}\right)=\operatorname{ord}_{v}\left(a_{i}-a_{j}\right)$ and $-\log _{v}\left(r_{i}\right)=$ 
$\operatorname{ord}_{v}\left(b_{i}\right)$, the system is

$$
\left\{\begin{array}{l}
\sum_{i=1}^{M} w_{i}=1, \quad \text { and for } j=1, \ldots, M \\
\sum_{i=1, i \neq j}^{M} w_{i}\left(\operatorname{ord}_{v}\left(a_{i}-a_{j}\right)\right)+w_{j}\left(\operatorname{ord}_{v}\left(b_{j}\right)+\frac{1}{q_{v}-1}\right)=V\left(E_{v}\right) .
\end{array}\right.
$$

By the existence and uniqueness of $\mu$, this system has a unique solution, in which $w_{i}=\mu\left(E_{v, i}\right)$. Since the matrices have rational entries, $V\left(E_{v}\right)$ and the $w_{i}$ are rational. Clearly each $w_{i} \geq 0$; in fact, each $w_{i}>0$ since if $w_{j}=0$ for some $j$ then we would have $G\left(z, \infty ; E_{v}\right)>0$ on $E_{v, j}$. However, this is impossible since $G\left(z, \infty ; E_{v}\right)=0$ a.e. on $E_{v}$ (see [11, Theorem 4.1.11, p. 195]). As before,

$$
\gamma\left(E_{v}\right)=q_{v}^{-V\left(E_{v}\right)} .
$$

Thus, again, computation of capacities reduces to linear algebra. The equations (3.10) will play a key role in the proof of Theorem 2.3 (see Lemmas 8.7 and 8.8).

The definitions of the capacity for PL-domains and compact sets are consistent. In $[11, \S 4.3]$ it is shown that for a PL-domain $U_{v}$,

$$
\gamma\left(U_{v}\right)=\sup _{\substack{E_{v} \subset U_{v} \\ E_{v}=\text { compact }}} \gamma\left(E_{v}\right),
$$

while for a compact set $E_{v}$,

$$
\gamma\left(E_{v}\right)=\inf _{\substack{U_{v} \supset E_{v} \\ U_{v}=\text { PL-domain }}} \gamma\left(U_{v}\right) .
$$

4. Reductions. In this section, we will derive Theorem 2.2 from Theorem 2.1, and Theorem 2.1 from Theorem 2.3. We will also outline the strategy for the proof of Theorem 2.3.

Preliminary reductions. Before proving Theorems 2.1 and 2.3, we will make some adjustments to the sets $\mathbb{E}=\prod_{v} E_{v}$ and $\mathbb{U}=\prod_{v} U_{v}$. By assumption, each $E_{v}$ is stable under $\operatorname{Gal}_{\mathrm{c}}\left(\mathbb{C}_{v} / K_{v}\right)$ and each $U_{v}$ is open. We will arrange that in addition each $E_{v}$ is closed and has a simple form, and that each $U_{v}$ is stable under $\operatorname{Gal}_{\mathrm{c}}\left(\mathbb{C}_{v} / K_{v}\right)$. Since the theorems concern the existence of points $\alpha \in \widetilde{K}$ with conjugates in $\mathbb{U}$, the conclusions remain valid as long as we do not enlarge the sets $U_{v}$.

REDUCTION 1. We claim that we can assume that $\gamma(\mathbb{E})>1$. The original hypothesis of Theorem 2.1 was that either $\gamma(\mathbb{E})>1$, or that $\gamma(\mathbb{E})=1$ and at least one archimedean $E_{v}$ is compact. In the latter case, choose an 
archimedean place where $E_{v}$ is compact. If $K_{v} \cong \mathbb{C}$, let $Z_{v}$ be a closed ball in $U_{v}$ which belongs to the unbounded component of $\mathbb{C} \backslash E_{v}$. If $K_{v} \cong \mathbb{R}$, let $Z_{v}$ be a closed interval in $\mathbb{R} \cap U_{v}$ which belongs to $\mathbb{C} \backslash E_{v}$. Then $\gamma\left(E_{v} \cup Z_{v}\right)>\gamma\left(E_{v}\right)$, so by replacing $E_{v}$ with $E_{v} \cup Z_{v}$ we can make $\gamma(\mathbb{E})>1$.

REDUCTION 2. Let $S$ be the distinguished set of places where $E_{v} \subset$ $\mathbb{P}^{1}\left(L_{w}\right)$ for a finite galois $L_{w} / K_{v}$; we can assume $S$ contains all archimedean $v$. We claim that we can assume that for each $v \in S, E_{v}$ is compact; and for each nonarchimedean $v \in S, E_{v}$ has the form

$$
E_{v}=\bigcup_{i=1}^{M} a_{i}+\pi_{w}^{n_{i}} O_{w},
$$

where $O_{w}$ is the ring of integers of $L_{w}$. Furthermore, we claim we can assume that for each $v \in S, U_{v}$ is stable under $\operatorname{Gal}_{\mathrm{c}}\left(\mathbb{C}_{v} / K_{v}\right)$, and for each nonarchimedean $v \in S, E_{v}=L_{w} \cap U_{v}$.

Let $\widehat{S}$ be the finite set of places of $K$ consisting of all the archimedean places and all the nonarchimedean places where $E_{v} \neq \widehat{O}_{v}$; clearly $S \subset \widehat{S}$. Since

$$
\prod_{v \in \widehat{S}} \gamma\left(E_{v}\right)^{D_{v}}=\gamma(\mathbb{E})>1
$$

and since

$$
\gamma\left(E_{v}\right)=\sup _{\substack{F_{v} \subset E_{v} \\ F_{v} \text { compact }}} \gamma\left(F_{v}\right),
$$

for each $v \in \widehat{S}$ we can choose a compact $F_{v} \subset E_{v}$ such that

$$
\prod_{v \in \widehat{S}} \gamma\left(F_{v}\right)^{D_{v}}>1
$$

However, a priori $F_{v}$ may not be stable under $\operatorname{Gal}_{\mathrm{c}}\left(\mathbb{C}_{v} / K_{v}\right)$.

In the archimedean case, if $K_{v} \cong \mathbb{C}$, replace $E_{v}$ by $F_{v}$; if $K_{v} \cong \mathbb{R}$, let $\sigma$ be complex conjugation, and replace $E_{v}$ by $F_{v} \cup \sigma\left(F_{v}\right)$ and $U_{v}$ by $U_{v} \cap \sigma\left(U_{v}\right)$.

In the nonarchimedean case, for $v$ in $S$, since $U_{v}$ is open, for each $x \in E_{v}$ there is a ball $B\left(x, r_{x}\right) \subset U_{v}$. Since $E_{v} \subset L_{w}$ is stable under $\operatorname{Gal}\left(L_{w} / K_{v}\right)$, and $\operatorname{Gal}\left(L_{w} / K_{v}\right)$ is finite, we can assume $r_{x}$ is small enough that $B\left(\sigma x, r_{x}\right) \subset$ $U_{v}$ for all $\sigma \in \operatorname{Gal}\left(L_{w} / K_{v}\right)$; after further reducing $r_{x}$, we can assume that $r_{x}$ belongs to the value group of $L_{w}^{\times}$. $F_{v}$ is compact, so we can cover it by a finite number of balls $B\left(x, r_{x}\right)$. Replacing $U_{v}$ by the union of these balls and their conjugates under $\operatorname{Gal}\left(L_{w} / K_{v}\right)$, and then replacing $E_{v}$ by $L_{w} \cap U_{v}$ (which contains $F_{v}$ ), yields all the claims. 
REDUCTION 3. We claim that for each nonarchimedean $v \in \widehat{S}$, but not in $S$, we can assume that $E_{v}$ is a PL-domain of the form

$$
E_{v}=\bigcup_{i=1}^{M} B\left(a_{i}, r_{i}\right),
$$

and that $U_{v}=E_{v}$; in particular $U_{v}$ is stable under $\operatorname{Gal}_{\mathrm{c}}\left(\mathbb{C}_{v} / K_{v}\right)$.

For $v \notin S$ the set $F_{v}$ constructed above need not be contained in a finite extension $L_{w} / K_{v}$, and may have infinitely many conjugates under $\operatorname{Gal}_{\mathrm{c}}\left(\mathbb{C}_{v} / K_{v}\right)$. However, we will show that $F_{v}$ is contained in a Galois-stable set of the form (4.2), contained in $U_{v}$. Fixing $x \in F_{v}$, and using the fact that $x \in F_{v} \subset E_{v}$, the Galois-stability of $E_{v}$ means that $\sigma x \in E_{v}$ for each $\sigma \in \operatorname{Gal}_{\mathrm{c}}\left(\mathbb{C}_{v} / K_{v}\right)$. Since $U_{v}$ is open, for each $\sigma$ there is an $r>0$ such that $B(\sigma x, r)^{-} \subset U_{v}$. Put

$$
r_{\sigma}=\sup _{B(\sigma x, r)^{-} \subset U_{v}} r
$$

so that $B\left(\sigma x, r_{\sigma}\right)^{-} \subset U_{v}$. The map

$$
\varphi_{x}: \operatorname{Gal}_{\mathrm{c}}\left(\mathbb{C}_{v} / K_{v}\right) \rightarrow \mathbb{R}, \quad \varphi_{x}(\sigma)=r_{\sigma}
$$

is continuous and locally constant, since for a given $\sigma_{0} \in \operatorname{Gal}_{\mathrm{c}}\left(\mathbb{C}_{v} / K_{v}\right)$, if $\sigma x \in B\left(\sigma_{0} x, r_{\sigma_{0}}\right)^{-}$, the ultrametric inequality shows that $r_{\sigma}=r_{\sigma_{0}}$. Since $\operatorname{Gal}_{\mathrm{c}}\left(\mathbb{C}_{v} / K_{v}\right)$ is compact, there is an $r>0$ such that $B(\sigma x, r) \subset U_{v}$ for all $\sigma \in \operatorname{Gal}_{\mathrm{c}}\left(\mathbb{C}_{v} / K_{v}\right)$. Without loss of generality, take $r$ in the value group of $\mathbb{C}_{v}^{\times}$. Fix $\alpha \in \widetilde{K}_{v} \cap B(x, r)$. The fact that $\operatorname{Gal}_{\mathrm{c}}\left(\mathbb{C}_{v} / K_{v}\right)$ preserves $|x|_{v}$ means that $B(\sigma x, r)=B(\sigma \alpha, r)$ for all $\sigma$. Since $\alpha$ has only finitely many conjugates, there are only finitely many distinct balls $B(\sigma x, r)$.

Since $F_{v}$ is compact, we can cover it a finite number of balls $B(x, r)$. Replacing $E_{v}$ by the union of these balls and their conjugates, we obtain (4.2). This set is open and contained in $U_{v}$, so we can replace $U_{v}$ with it as well; by construction, it is stable under $\operatorname{Gal}_{\mathrm{c}}\left(\mathbb{C}_{v} / K_{v}\right)$.

ReduCtion 4. Finally, we claim that for each $v \notin \widehat{S}$, we can assume that

$$
E_{v}=U_{v}=\widehat{O}_{v}
$$

Since $v \notin \widehat{S}$, by hypothesis we have $E_{v}=\widehat{O}_{v}$; after shrinking $U_{v}$, we can assume that $U_{v}=\widehat{O}_{v}$ as well.

Proof of Theorem 2.2, assuming Theorem 2.1. Let $\mathbb{E}=\prod_{v} E_{v}$ satisfy the hypotheses of Theorem 2.2. We will construct sets $E_{v}^{\prime} \subset U_{v}^{\prime} \subset E_{v}$ such that $\mathbb{E}^{\prime}=\prod_{v} E_{v}^{\prime}$ and $\mathbb{U}^{\prime}=\prod_{v} U_{v}^{\prime}$ satisfy the hypotheses of Theorem 2.1. By hypothesis, for each nonarchimedean $v$, either $E_{v}$ is a union of balls and hence is open in the $v$-adic topology, so we can take $E_{v}^{\prime}=U_{v}^{\prime}=E_{v}$; or $E_{v}=\bigcup_{i=1}^{M}\left(a_{i}+b_{i} O_{w}\right)$, so we can take $v \in S$, and put $E_{v}^{\prime}=E_{v}, U_{v}^{\prime}=$ 
$\bigcup_{i=1}^{M} B\left(a_{i},\left|b_{i}\right|_{v}\right)$. For each archimedean $v$ with $K_{v} \cong \mathbb{R}$ and $E_{v} \subset \mathbb{R}, E_{v}$ is a finite union of closed intervals; let $E_{v}^{\prime}=U_{v}^{\prime}$ be the (real) interior of $E_{v}$. Since $U_{v}^{\prime}$ is obtained from $E_{v}$ by removing a finite set of points, which is a set of capacity 0 , Theorem III.18 of [13] shows that $\gamma\left(E_{v}^{\prime}\right)=\gamma\left(E_{v}\right)$. For each archimedean $v$ with $K_{v} \cong \mathbb{C}$ or with $K_{v} \cong \mathbb{R}$ but $E_{v} \nsubseteq \mathbb{R}$, let $E_{v}^{\prime}=U_{v}^{\prime}$ be the (complex) interior of $E_{v}$. Since $E_{v}$ is the closure of $U_{v}^{\prime}$, Lemma 11.1 of Appendix I shows that $\gamma\left(E_{v}^{\prime}\right)=\gamma\left(E_{v}\right)$. Each $E_{v}^{\prime}$ is Galois-stable since $E_{v}$ is Galois-stable; each $U_{v}^{\prime}$ is open; and

$$
\prod_{v} \gamma\left(E_{v}^{\prime}\right)^{D_{v}}=\prod_{v} \gamma\left(E_{v}\right)^{D_{v}}>1 .
$$

Thus, we can apply Theorem 2.1 to $\mathbb{E}^{\prime}$ and $\mathbb{U}^{\prime}$.

Proof of Theorem 2.1, assuming Theorem 2.3. Suppose the hypotheses of Theorem 2.1 hold, and let $S, \widehat{S}$ be as above.

The global capacity $\gamma(\mathbb{E})$ has good functoriality under base change: by [11, Theorem 5.1 .13 , p. 333], for any finite extension $L / K$, we can pull back $\mathbb{E}=\mathbb{E}_{K}$ to a set $\mathbb{E}_{L}$ for which

$$
\gamma\left(\mathbb{E}_{L}\right)=\gamma\left(\mathbb{E}_{K}\right)^{[L: K]}
$$

$\mathbb{E}_{L}$ is defined as follows: given a place $w$ of $L$, let $v$ be the place of $K$ below $w$. Fix an isomorphism of $\mathbb{C}_{w}$ with $\mathbb{C}_{v}$, and use it to define a set $E_{w} \cong E_{v}$. Since $E_{v}$ is stable under $\mathrm{Gal}_{c}\left(\mathbb{C}_{v} / K_{v}\right), E_{w}$ is independent of the choice of isomorphism, and is stable under $\mathrm{Gal}_{\mathrm{c}}\left(\mathbb{C}_{w} / L_{w}\right)$. If we put $\mathbb{E}_{L}=\prod_{w} E_{w}$, then (4.3) holds.

After our preliminary reductions, we can assume that each $U_{v}$ is stable under $\operatorname{Gal}_{c}\left(\mathbb{C}_{v} / K_{v}\right)$, so we can also pull back $\mathbb{U}=\mathbb{U}_{K}$ to a well-defined adelic neighborhood $\mathbb{U}_{L}$ of $\mathbb{E}_{L}$.

We claim that by replacing $K$ with an appropriate finite Galois extension $L / K$, we can assume that $E_{v} \cap K_{v}$ is nonempty for each $v$, and that $K_{v}=L_{w}$ for each $v \in S$. We will now construct $L$. For each $v \in S$ we are given a finite Galois extension $L_{w} / K_{v}$ with $E_{v} \subset L_{w}$. For each $v \in \widehat{S} \backslash S$, choose a finite Galois $L_{w} / K_{v}$ such that $E_{v} \cap L_{w} \neq \emptyset$. Let $D$ be the least common multiple of the $\left[L_{w}: K_{v}\right]$, and put $m_{v}=D /\left[L_{w}: K_{v}\right]$. For each $v$, choose $m_{v}$ distinct, nonconjugate primitive elements $\alpha_{v, j}$ for $L_{w} / K_{v}$. Let $f_{v, j}(z) \in K_{v}[z]$ be the minimal polynomial for $\alpha_{v, j}$, and put

$$
f_{v}(z)=\prod_{j=1}^{m_{v}} f_{v, j}(z) .
$$

By the weak approximation theorem, we can find a monic $f(x) \in K[x]$ which approximates each $f_{v}(x)$ so closely that each root of $f(x)$ in $\mathbb{C}_{v}$ is again a primitive element for $L_{w} / K_{v}$ (continuity of the roots, plus Krasner's lemma). We can also arrange that $f(x)$ be irreducible, by requiring it to be 
an Eisenstein polynomial at some fixed $v_{0} \notin \widehat{S}$. Let $L$ be the splitting field of $f(x)$ over $K$. Then $L / K$ is a finite Galois extension, with the prescribed completion $L_{w}$ at each place $w \mid v$, for each $v \in \widehat{S}$.

We can now apply Theorem 2.3 to $\mathbb{E}_{L}$ and $\mathbb{U}_{L}$. If $\alpha \in \widetilde{L}=\widetilde{K}$ is a number whose conjugates over $L$ (in $\mathbb{C}_{w}$ ) belong to $U_{w}$ for each $w$, then because each $U_{v}$ is stable under $\operatorname{Gal}_{c}\left(\mathbb{C}_{v} / K_{v}\right)$ and $U_{w}=U_{v}$ for each $w \mid v$, the conjugates of $\alpha$ over $K$ (in $\mathbb{C}_{v}$ ) belong to $U_{v}$, for each $v$. Thus, without loss of generality, we can replace $K$ by $L$, and Theorem 2.1 follows from Theorem 2.3.

Outline of the proof of Theorem 2.3. The strategy is to show that under the hypotheses of Theorem 2.3, for each sufficiently large integer $n$ it is possible to find an irreducible monic polynomial $u(z) \in K[z]$ whose roots, for each $v \in \widehat{S}$, belong to $U_{v}$; whose coefficients are integral at all $v \notin \widehat{S}$; and which has the desired ramification at the places in $S^{\prime}=S_{1}^{\prime} \cup S_{2}^{\prime}$. We can assume that the preliminary reductions for Theorem 2.1 have been carried out, so that $\gamma(\mathbb{E})>1$ and the sets $E_{v}$ are of restricted types. The roots of the $u(z)$ will be the algebraic numbers required by the theorem.

The polynomials $u(z)$ will be constructed by "patching together" local polynomials $u_{v}^{(0)}(z) \in K_{v}[z]$, for each $v \in \widehat{S} \cup S^{\prime}$. The patching process has both a local and a global aspect.

For each $v$, the local part of the patching process consists of choosing a monic polynomial $u_{v}^{(0)}(z) \in K_{v}[z]$ of degree $n$ which has all its roots in $U_{v}$ (and in $K_{v}$, if $v \in S$ ), and successively replacing it by polynomials $u_{v}^{(1)}(z), u_{v}^{(2)}(z), \ldots, u_{v}^{(n)}(z)$, where $u_{v}^{(k)}(z)$ has the same $k-1$ highestorder coefficients as $u_{v}^{(k-1)}(z)$, and whose $k$ th coefficient differs from that of $u_{v}^{(k-1)}(z)$ by a specified quantity $\Delta_{v}^{(k)} \in K_{v}$; its lower-order coefficients may be changed in arbitrary ways. (We will call this "patching with numbers

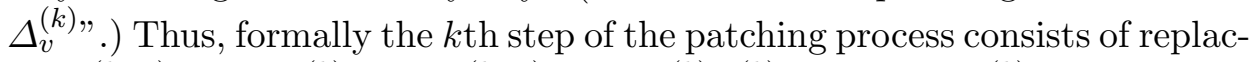
ing $u_{v}^{(k-1)}(z)$ by $u_{v}^{(k)}(z)=u_{v}^{(k-1)}(z)+\Delta_{v}^{(k)} w_{v}^{(k)}(z)$, where $w_{v}^{(k)}(z) \in K_{v}[z]$ is monic of degree $n-k$. In the following sections, we will show that if $u_{v}^{(0)}(z)$ is chosen carefully and the $\Delta_{v}^{(k)}$ are subject to appropriate constraints, namely that for certain constants $B_{v}, h_{v}$ and $L$

$$
\left|\Delta_{v}^{(k)}\right|_{v} \leq \begin{cases}B_{v} & \text { for } k \leq L, \\ h_{v}^{k} & \text { for } k>L,\end{cases}
$$

then the $w_{v}^{(k)}(z)$ can be chosen so that each $u_{v}^{(k)}(z)$ has all of its roots in $U_{v}$ (and in $K_{v}$, if $v \in S$ ).

From a global point of view, the patching process consists of choosing the numbers $\Delta_{v}^{(k)}$ in such a way that in the $k$ th step, the coefficient $c_{v, k}^{(k)}$ 
of $z^{n-k}$ in each $u_{v}^{(k)}(z)$ becomes a global $\widehat{S}$-integer $c_{k} \in K$ independent of $v$. In practice, we first choose the target coefficient $c_{k}$, and if $c_{v, k}^{(k-1)}$ is the coefficient of $z^{n-k}$ in $u_{v}^{(k-1)}(z)$ we then take

$$
\Delta_{v}^{(k)}=c_{k}-c_{v, k}^{(k-1)} .
$$

The burden of the global part of the argument is to show that numbers $c_{k}$ can be found so that the local conditions (4.4) are satisfied. This will be a consequence of the Strong Approximation Theorem. At the end of the patching process, all the $u_{v}^{(n)}(z)$ will be equal to a single global polynomial $u(z) \in K[z]$. This polynomial $u(z)$ will therefore have all its roots in $U_{v}$ (and in $K_{v}$, if $v \in S$ ) for all $v \in \widehat{S}$, and will satisfy the desired ramification properties, for $v \in S^{\prime}$. Since its coefficients are $\widehat{S}$-integers, its roots also belong to $U_{v}=\widehat{O}_{v}$ for all $v \notin \widehat{S}$.

We will provide the details of the proof after presenting the local patching constructions. However, to give some perspective on the argument, we indicate how the various parameters are related.

By Reduction 1, $\prod_{v \in \widehat{S} \cup S^{\prime}} \gamma\left(E_{v}\right)^{D_{v}}=\gamma(\mathbb{E})>1$. For each $v \in \widehat{S} \cup S^{\prime}$, fix numbers $0<h_{v}<r_{v}<\gamma\left(E_{v}\right)$ subject to the condition $\prod_{v \in \widehat{S} \cup S^{\prime}} h_{v}^{D_{v}}>1$. The numbers $h_{v}$ and $r_{v}$ control the freedom in the local patching processes.

In the construction, we will choose a constant $L$, the number of coefficients to be deemed "high-order". $L$ is subject to a finite number of local constraints depending only on $E_{v}, U_{v}, h_{v}$ and $r_{v}$, for each $v$, and also to a global constraint arising from the Strong Approximation Theorem; each of these constraints is satisfied for all large $L$.

After $L$ has been fixed, we consider the numbers $B_{v}$. For nonarchimedean $v$, the $B_{v}$ are determined by the local patching process and depend on $L$. However, for archimedean $v$, the $B_{v}$ can be chosen as large as we wish, provided $n$ is big enough. If we make $\prod_{v \in \widehat{S} \cup S^{\prime}} B_{v}^{D_{v}}$ sufficiently large, the global patching process can be carried out. In this way the construction succeeds for all sufficiently large $n$.

Sections 5 through 9 contain the local patching constructions. Section 10 gives the global patching argument.

5. Local patching for sets in $\mathbb{C}$. When $K_{v} \simeq \mathbb{C}$, or when $K_{v} \simeq \mathbb{R}$ and $E_{v}$ is stable under complex conjugation, our patching process is an extension of a method going back to Fekete and Szegö [5].

Proposition 5.1. Suppose that $K_{v}$ is archimedean, and that $E_{v} \subset U_{v}$ $\subset \mathbb{C}$, where $E_{v}$ is compact and $U_{v}$ is open. There is a number $M_{v}$, depending only on $E_{v}$ and $U_{v}$, with the following property. Let $0<h_{v}<r_{v}<\gamma\left(E_{v}\right)$ be 
given, and let $L$ be an integer satisfying

$$
\left(\frac{h_{v}}{r_{v}}\right)^{L} \frac{M_{v}}{1-h_{v} / r_{v}}<\frac{1}{8}
$$

If $K_{v} \simeq \mathbb{C}$ (resp. if $K_{v} \simeq \mathbb{R}, E_{v}$ is stable under complex conjugation, and $E_{v} \cap \mathbb{R}$ is nonempty) then, for any $B_{v}>0$, for each sufficiently large $n$ there is a monic polynomial $u_{v}^{(0)}(z) \in \mathbb{C}[z]$ (resp. $\mathbb{R}[z]$ ) of degree $n$, whose roots belong to $U_{v}$ and which can be patched with arbitrary $\Delta_{v}^{(k)} \in \mathbb{C}$ (resp. $\left.\mathbb{R}\right)$ satisfying

$$
\left|\Delta_{v}^{(k)}\right| \leq \begin{cases}B_{v} & \text { for } k \leq L \\ h_{v}^{k} & \text { for } k>L\end{cases}
$$

in such a way that its roots remain in $U_{v}$.

Proof. We will give the construction when $K_{v} \simeq \mathbb{C}$, noting minor changes in the argument when $K_{v} \simeq \mathbb{R}$.

We first reduce to the case where $E_{v}$ is a finite union of arcs, and $\mathbb{C} \backslash E_{v}$ is connected. To achieve this, first replace $E_{v}$ with a finite number of closed balls contained in $U_{v}$, whose interiors cover $E_{v}$; this only increases $\gamma\left(E_{v}\right)$. Let $\partial E_{v}^{\text {out }}$ be the outer boundary of $E_{v}$; as noted earlier, $\gamma\left(E_{v}\right)=\gamma\left(\partial E_{v}^{\text {out }}\right)$. Replacing $E_{v}$ by $\partial E_{v}^{\text {out }}$, we can assume that $E_{v}$ consists of a finite union of $\operatorname{arcs} \bigcup_{i=1}^{M} F_{i}$. For each $i$, choose a point $a_{i} \in F_{i}$, and let $B\left(a_{i}, r\right)^{-}$be the open ball of radius $r$ centered at $a_{i}$. For each $i$, put $e_{i}(r)=E_{v} \cap B\left(a_{i}, r\right)^{-}$, and put

$$
E_{v}(r):=E_{v} \backslash\left(\bigcup_{i=1}^{M} B\left(a_{i}, r\right)^{-}\right)
$$

so that $E_{v}=E_{v}(r) \cup\left(\bigcup_{i=1}^{M} e_{i}(r)\right)$. Then $\mathbb{C} \backslash E_{v}(r)$ is connected, and if $r$ is small enough, no component of $E_{v}(r)$ is reduced to a point. Fix $R$ large enough that $E_{v} \subset B(0, R)$. By [13, Theorem III.17, p. 63] and the homogeneity of $\gamma(E)$,

$$
\frac{1}{\log \left(2 R / \gamma\left(E_{v}\right)\right)} \leq \frac{1}{\log \left(2 R / \gamma\left(E_{v}(r)\right)\right)}+\sum_{i=1}^{M} \frac{1}{\log \left(2 R / \gamma\left(e_{i}(r)\right)\right)}
$$

Furthermore, $\gamma\left(e_{i}(r)\right) \leq \gamma\left(B\left(a_{i}, r\right)\right)=r$. Since $r_{v}>0$ is fixed and $\gamma\left(E_{v}\right)>$ $r_{v}$, if $r$ is small enough the inequality above implies that $\gamma\left(E_{v}(r)\right)>r_{v}$. Replacing $E_{v}$ by $E_{v}(r)$ for a suitably small $r$ yields a set with the properties we need. If $K_{v} \simeq \mathbb{R}$, the construction can be carried out in a way stable under complex conjugation.

After these reductions the Green function $G\left(z, \infty ; E_{v}\right)$ is continuous, with $G\left(z, \infty ; E_{v}\right)=0$ for all $z \in E_{v}$, and $G\left(z, \infty ; E_{v}\right)>0$ for all $z \notin E_{v}$. By 
continuity, there is an $\varepsilon>0$ such that

$$
\left\{z \in \mathbb{C}: G\left(z, \infty ; E_{v}\right) \leq \varepsilon\right\} \subset U_{v} .
$$

Put

$$
\begin{aligned}
\Omega_{\varepsilon} & =\left\{z \in \mathbb{C}: G\left(z, \infty ; E_{v}\right)<\varepsilon\right\}, \\
\partial \Omega_{\varepsilon} & =\left\{z \in \mathbb{C}: G\left(z, \infty ; E_{v}\right)=\varepsilon\right\} .
\end{aligned}
$$

Then $\Omega_{\varepsilon}$ is open, and $E_{v} \subset \Omega_{\varepsilon} \subset U_{v}$.

We can approximate the equilibrium distribution $\mu$ of $E_{v}$ by a discrete measure $\sum_{i=1}^{n_{v}}\left(1 / n_{v}\right) \delta_{\alpha_{i}}(z)$ sufficiently well that the monic polynomial

$$
g(z)=\prod_{i=1}^{n_{v}}\left(z-\alpha_{i}\right) \in \mathbb{C}[z]
$$

has all its roots in $E_{v}$, and for all $z \notin \Omega_{\varepsilon}$ it satisfies

$$
\left|\frac{1}{n_{v}} \log _{v}(|g(z)|)-\left(G\left(z, \infty ; E_{v}\right)-V\left(E_{v}\right)\right)\right|<\frac{\varepsilon}{2}
$$

(see [6, pp. 294-295], or [11, Lemma 3.3.5, p. 169]). When $K_{v} \simeq \mathbb{R}, \mu$ is stable under complex conjugation, and $g(z)$ can be chosen in $\mathbb{R}[z]$. This polynomial $g(z)$, and its degree $n_{v}$, will be fixed for the rest of the construction. Put $R_{v}=\gamma\left(E_{v}\right)$.

Fix a point $a \in E_{v}$ (if $K_{v} \simeq \mathbb{R}$, choose $a \in E_{v} \cap \mathbb{R}$ ), and put

$$
\begin{aligned}
& C_{v}^{-}=\min _{0 \leq h<n_{v}}\left(\left(\min _{z \in \partial \Omega_{\varepsilon}}\left|(z-a)^{h}\right|\right) /\left(R_{v} e^{2 \varepsilon}\right)^{h}\right), \\
& C_{v}^{+}=\max _{0 \leq h<n_{v}}\left(\left(\max _{z \in \partial \Omega_{\varepsilon}}\left|(z-a)^{h}\right|\right) / R_{v}^{h}\right) .
\end{aligned}
$$

Finally, put

$$
M_{v}=C_{v}^{+} / C_{v}^{-} .
$$

This is the constant in the proposition.

Construction of the initial patching polynomials. The condition imposed by $v$ on the globally chosen constant $L$ will be that

$$
\left(\frac{h_{v}}{r_{v}}\right)^{L} \frac{M_{v}}{1-h_{v} / r_{v}}<\frac{1}{8}
$$

This holds for all sufficiently large $L$. Suppose $L$ has been fixed, and choose an integer $N_{v}$ large enough that $n_{v} N_{v}>L$. Set

$$
\widetilde{g}(z)=g(z)^{N_{v}} \text {. }
$$

By continuity there is a $\delta>0$ such that for any monic $\widehat{g}(z) \in \mathbb{C}[z]$ (resp. $\mathbb{R}[z]$ ) of degree $n_{v} N_{v}$, whose coefficients differ from those of $\widetilde{g}(z)$ by less than $\delta$ in absolute value, all the roots of $\widehat{g}(z)$ belong to $\Omega_{\varepsilon}$, and for all $z \in \partial \Omega_{\varepsilon}$

$$
\left|\frac{1}{n_{v} N_{v}} \log _{v}(|\widehat{g}(z)|)-\frac{1}{n_{v}} \log _{v}(|g(z)|)\right|<\min \left(\frac{\varepsilon}{2}, \frac{\log _{v}(2)}{n_{v} N_{v}}\right) .
$$


If $\widehat{g}(z)$ is such a polynomial, then $\frac{1}{n_{v} N_{v}} \log _{v}(|\widehat{g}(z)|)-\frac{1}{n_{v}} \log _{v}(|g(z)|)$ is harmonic in the complement of $\Omega_{\varepsilon}$ and remains bounded as $z \rightarrow \infty$. By (5.8) and the maximum modulus principle for harmonic functions, (5.8) holds for all $z \notin \Omega_{\varepsilon}$. From (5.4) and (5.8), and from $R_{v}=e^{-V\left(E_{v}\right)}$, it follows that

$$
|\widehat{g}(z)|>R_{v}^{n_{v} N_{v}} \quad \text { for all } z \notin \Omega_{\varepsilon} .
$$

Given $n$, to construct the initial polynomial $u_{v}^{(0)}(z)$, first write $n=s+$ $m \cdot n_{v} N_{v}$, where $s, m$ are integers and $0 \leq s<n_{v} N_{v}$. Decompose $s=s_{1}+$ $s_{2} \cdot n_{v}$ where $0 \leq s_{1}<n_{v}$ and $0 \leq s_{2} \leq N_{v}$, and put

$$
f_{s}(z)=(z-a)^{s_{1}} g(z)^{s_{2}} .
$$

Then, put

$$
u_{v}^{(0)}(z)=f_{s}(z) \widetilde{g}(z)^{m} .
$$

Note that $u_{v}^{(0)}(z)$ has all its zeros in $\Omega_{\varepsilon}$. For the rest of the construction, we hold $s$ fixed, and view $n$ as a function of $m$. It suffices to prove the proposition for each fixed $s$, and all sufficiently large $m$.

PHASE 1. Patching the high-order coefficients $(k \leq L)$. We will patch the high-order coefficients of $u_{v}^{(0)}(z)$ by sequentially modifying the coefficients of $\widetilde{g}(z)$, taking advantage of a phenomenon of "magnification". This idea is new, and is what ultimately enables archimedean and nonarchimedean polynomials to be patched together.

We can assume $m$ is large enough that $m \delta>B_{v}$, where $B_{v}$ is the number specified in the proposition.

Put $\widehat{g}^{(0)}(z)=\widetilde{g}(z)$. For $k=1, \ldots, L$, we will inductively construct a polynomial $\widehat{g}^{(k)}(z)$ by adding a number $\delta_{k} \in \mathbb{C}\left(\right.$ resp. $\left.\delta_{k} \in \mathbb{R}\right)$ with $\left|\delta_{k}\right|<\delta$, to the $k$ th coefficient of $\widehat{g}^{(k-1)}(z)$; we will then put

$$
u_{v}^{(k)}(z)=f_{s}(z) \widehat{g}^{(k)}(z)^{m} .
$$

Since $\left|\delta_{k}\right|<\delta$, the discussion above applies to each $\widehat{g}^{(k)}(z)$. Fixing $k$, write

$$
f_{s}(z)=z^{s}+\sum_{h=1}^{s} a_{h} z^{s-h}, \quad \widehat{g}^{(k-1)}(z)=z^{n_{v} N_{v}}+\sum_{j=1}^{n_{v} N_{v}} b_{j} z^{d-j}
$$

and expand

$$
u_{v}^{(k-1)}(z):=f_{s}(z) \widehat{g}^{(k-1)}(z)^{m}=z^{n}+\sum_{j=1}^{n} c_{v, j} z^{n-j} .
$$

By the multinomial theorem (since $n_{v} N_{v}>L$ and $k \leq L$ ), $c_{v, k}$ has the form

$$
c_{v, k}=\sum_{h, l_{0}, l_{1}, \ldots, l_{k}}\left(\begin{array}{c}
m \\
l_{0} l_{1} \ldots l_{k}
\end{array}\right) a_{h} b_{1}^{l_{1}} \ldots b_{k}^{l_{k}}
$$


where the sum is over all integers $h, l_{0}, l_{1}, \ldots, l_{k} \geq 0$ satisfying

$$
h+l_{1}+2 l_{2}+\ldots+k l_{k}=k, \quad l_{0}+l_{1}+\ldots+l_{k}=m, \quad 0 \leq h \leq s .
$$

Note that there are only a bounded number of possibilities for $h, l_{1}, \ldots, l_{k}$, independent of $m$; and if $m>L$ and a choice of $h, l_{1}, \ldots, l_{k}$ is given we can take $l_{0}=m-l_{1}-\ldots-l_{k}$. Moreover, there is only one term in (5.10) in which $b_{k}$ appears, namely the one with $h=l_{1}=\ldots=l_{k-1}=0, l_{k}=1$. For that term, the multinomial coefficient is $m$, and $a_{0}=1$. Thus, for sufficiently large $m, c_{v, k}$ is a polynomial in $m$ and $b_{1}, \ldots, b_{k}$ of the form

$$
c_{v, k}=m b_{k}+Q_{k, s}\left(m, b_{1}, \ldots, b_{k-1}\right),
$$

which depends linearly on $b_{k}$.

If we define $\widehat{g}^{(k)}(z)$ by adding $\delta_{k}$ to the coefficient $b_{k}$ in $\widehat{g}^{(k-1)}(z)$, the coefficients $c_{v, l}$ in $u_{v}^{(k-1)}(z)$ with $l<k$ remain unchanged, $c_{v, k}$ is changed to $c_{v, k}+m \delta_{k}$, and the coefficients $c_{v, l}$ with $l>k$ are modified in ways that are unimportant. Thus, since $m \delta>B_{v}$, for $k=1, \ldots, L$ we can vary the $k$ th coefficient of $u^{(k-1)}(z)$ by a quantity $\Delta_{v, k}$ up to $B_{v}$ in magnitude, by adjusting the $k$ th coefficient of $\widehat{g}^{(k-1)}(z)$ by $\delta_{k}=\Delta_{v, k} / m$, a quantity at most $\delta$ in magnitude.

The $\Delta_{v, k}$ will be chosen on the basis of global considerations; for the remainder of the proof we assume that $\Delta_{v, 1}, \ldots, \Delta_{v, L}$ have been fixed, and write $\widehat{g}(z)$ for the corresponding polynomial $\widehat{g}^{(L)}(z)$. In the discussion below, we use only that $\widehat{g}(z)$ satisfies (5.8) (hence also (5.9)), and that it is monic with degree $n_{v} N_{v}$.

Phase 2. Patching the low-order coefficients $(L<k \leq n)$. We begin this phase of the patching process with

$$
u_{v}^{(L)}(z)=f_{s}(z) \widehat{g}(z)^{m},
$$

where $\widehat{g}(z)=\widehat{g}^{(L)}(z)$. Since $f_{s}(z)$ and $\widehat{g}(z)$ have all their zeros in $\Omega_{\varepsilon}$, the same is true for $u_{v}^{(L)}(z)$. Moreover, by (5.5) and (5.9),

$$
\left|u_{v}^{(L)}(z)\right|>C_{v}^{-} R_{v}^{n} \quad \text { for all } z \notin \Omega_{\varepsilon} .
$$

In the $k$ th patching step, for $k>L$, let $w_{v}^{(k)}(z)$ be the monic polynomial of degree $n-k$ defined by

$$
w_{v}^{(k)}(z)=(z-a)^{h_{1}} g(z)^{h_{2}} \widehat{g}(z)^{h_{3}},
$$

where the integers $h_{1}, h_{2}, h_{3}$ are determined by

$$
\begin{gathered}
\quad n-k=h_{1}+h_{2} n_{v}+h_{3} n_{v} N_{v}, \\
0 \leq h_{1}<n_{v}, \quad 0 \leq h_{2}<N_{v}, \quad 0 \leq h_{3}<m .
\end{gathered}
$$

We define $u_{v}^{(k)}(z)$ by setting

$$
u_{v}^{(k)}(z)=u_{v}^{(k-1)}(z)+\Delta_{v, k} w_{v}^{(k)}(z) .
$$


This has the effect of changing the $k$ th coefficient $c_{v, k}$ of $u_{v}^{(k-1)}(z)$ to $c_{v, k}+$ $\Delta_{v, k}$ in $u_{v}^{(k)}(z)$, and leaving the coefficients $c_{v, l}$ with $l<k$ unchanged. The $\Delta_{v, k}$ will be chosen on the basis of global considerations, subject to $\left|\Delta_{v, k}\right|$ $\leq h_{v}^{k}$.

We need bounds for $\left|w_{v}^{(k)}(z)\right|$ and $\left|u_{v}^{(L)}(z)\right|$ in terms of $|\widehat{g}(z)|$. First, we bound $|z-a|^{h}$, for $h=0, \ldots, n_{v}-1$. By (5.5), on $\partial \Omega_{\varepsilon}$,

$$
C_{v}^{-}\left(R_{v} e^{2 \varepsilon}\right)^{h} \leq|z-a|^{h} \leq C_{v}^{+} R_{v}^{h} .
$$

On the other hand by (5.4) and (5.8), on $\partial \Omega_{\varepsilon}$ also $R_{v} \leq|\widehat{g}(z)|^{1 /\left(n_{v} N_{v}\right)} \leq$ $R_{v} e^{2 \varepsilon}$. Thus

$$
C_{v}^{-}|\widehat{g}(z)|^{h /\left(n_{v} N_{v}\right)} \leq|z-a|^{h} \leq C_{v}^{+}|\widehat{g}(z)|^{h /\left(n_{v} N_{v}\right)} .
$$

By the maximum modulus principle for harmonic functions, applied to $\frac{h}{n_{v} N_{v}} \log _{v}(|\widehat{g}(z)|)-h \log (|z-a|)$ on $\mathbb{P}^{1}(\mathbb{C}) \backslash \Omega_{\varepsilon}$, these inequalities hold for all $z \notin \Omega_{\varepsilon}$. Second, we must bound $|g(z)|^{h}$, for $h=0, \ldots, N_{v}-1$. By (5.8), on $\partial \Omega_{\varepsilon}$ we have

$$
\frac{1}{2}|\widehat{g}(z)|^{h / N_{v}} \leq|g(z)|^{h} \leq 2|\widehat{g}(z)|^{h / N_{v}}
$$

and again by the maximum modulus principle, these inequalities hold for all $z \notin \Omega_{\varepsilon}$. Combining (5.14) and (5.15, we find that for all $z \notin \Omega_{\varepsilon}$,

$$
\begin{aligned}
& \left|w_{v}^{(k)}(z)\right|=\left|(z-a)^{h_{1}} g_{v}(z)^{h_{2}} \widehat{g}_{v}(z)^{h_{3}}\right| \leq 2 C_{v}^{+}|\widehat{g}(z)|^{(n-k) /\left(n_{v} N_{v}\right)}, \\
& \left|u_{v}^{(L)}(z)\right|=\left|(z-a)^{s_{1}} g(z)^{s_{2}} \widehat{g}(z)^{m}\right| \geq \frac{1}{2} C_{v}^{-}|\widehat{g}(z)|^{n /\left(n_{v} N_{v}\right)} .
\end{aligned}
$$

At the end of the construction we have

$$
u_{v}^{(n)}(z)=u_{v}^{(L)}(z)+\sum_{k=L+1}^{n} \Delta_{v, k} w_{v}^{(k)}(z) .
$$

By (5.16), (5.17), and (5.7), together with $M_{v}=C_{v}^{+} / C_{v}^{-}$and the inequalities $\left|\Delta_{v, k}\right| \leq h_{v}^{k}$ and $|\widehat{g}(z)|^{1 /\left(n_{v} N_{v}\right)}>R_{v}>r_{v}$, for all $z \notin \Omega_{\varepsilon}$ we have

$$
\begin{aligned}
\left|\frac{u_{v}^{(n)}(z)-u_{v}^{(L)}(z)}{u_{v}^{(L)}(z)}\right| & \leq 4 M_{v} \sum_{k=L+1}^{n}\left(h_{v}\right)^{k}|\widehat{g}(z)|^{-k /\left(n_{v} N_{v}\right)} \\
& <4 M_{v} \sum_{k=L}^{\infty}\left(\frac{h_{v}}{r_{v}}\right)^{k} \leq \frac{1}{2} .
\end{aligned}
$$

Finally from (5.19) and (5.12) we see that for all $z \notin \Omega_{\varepsilon}$,

$$
\left|u_{v}^{(n)}(z)\right| \geq \frac{1}{2}\left|u_{v}^{(L)}(z)\right|>\frac{1}{2} C_{v}^{-} R_{v}^{n}>0 .
$$

This means that $u_{v}^{(n)}(z)$ has all its zeros in $\Omega_{\varepsilon}$, and hence in $U_{v}$. 
6. Local patching for sets in $\mathbb{R}$. When $K_{v} \cong \mathbb{R}$ and $E_{v} \subset \mathbb{R}$, our patching process uses Chebyshev polynomials, extending a method due to Robinson [10]. Replacing $U_{v}$ by $U_{v} \cap \mathbb{R}$, we can assume $U_{v}$ is a real neighborhood of $E_{v}$.

Proposition 6.1. Suppose $K_{v} \cong \mathbb{R}$, and that $E_{v} \subset U_{v} \subset \mathbb{R}$, where $E_{v}$ is compact and $U_{v}$ is open. There is a number $M_{v}$, depending only on $E_{v}$ and $U_{v}$, with the following property. Let $0<h_{v}<r_{v}<\gamma\left(E_{v}\right)$ be given, and let $L$ be an integer such that

$$
\left(\frac{h_{v}}{r_{v}}\right)^{L} \frac{M_{v}}{1-h_{v} / r_{v}}<\frac{1}{16} .
$$

Given $B_{v}>0$, then for each sufficiently large $n$ there is a monic polynomial $u_{v}^{(0)}(z) \in \mathbb{R}[z]$ of degree $n$, whose roots belong to $U_{v}$ and which can be patched with arbitrary $\Delta_{v}^{(k)} \in \mathbb{R}$ satisfying

$$
\left|\Delta_{v}^{(k)}\right|_{v} \leq \begin{cases}B_{v} & \text { for } k \leq L, \\ h_{v}^{k} & \text { for } k>L,\end{cases}
$$

in such a way that its roots remain in $U_{v}$.

Proof. We begin with some preliminary reductions. After shrinking $U_{v}$, we can assume that it has compact closure, and after enlarging $E_{v}$ within $U_{v}$, we can assume that it is a finite union of closed intervals:

$$
E_{v}=\bigcup_{i=1}^{M}\left[a_{i}, b_{i}\right], \quad \text { where } \quad a_{1}<b_{1}<a_{2}<b_{2}<\ldots<a_{M}<b_{M} .
$$

Put $R_{v}=\gamma\left(E_{v}\right)$. By definition, the Chebyshev polynomial of degree $d$ for $E_{v}$ is a monic polynomial $T_{d}\left(z ; E_{v}\right) \in \mathbb{R}[z]$ of degree $d$, with minimal sup norm on $E_{v}$. It is known that such polynomials exist, and that

$$
\left\|T_{d}\left(z ; E_{v}\right)\right\|_{E_{v}} \geq 2 R_{v}^{d}
$$

Furthermore, since $E_{v}$ is a finite union of closed intervals, $T_{d}\left(z ; E_{v}\right)$ is unique, its roots are simple and belong to $\mathbb{R}$, and between each pair of roots, it achieves its maximum magnitude at a point of $E_{v}$. Its roots are real and lie in $\left[a_{1}, b_{M}\right]$, and there is at most one root in each "gap" $\left(b_{i}, a_{i+1}\right)$. For these facts, see e.g. [10].

Chebyshev polynomials for an interval $[-2 r, 2 r]$ are known explicitly, and are discussed for example in [9]. We will write $T_{d, r}(z)$ for $T_{d}(z ;[-2 r, 2 r])$; it is the polynomial defined by

$$
T_{d, r}(2 r \cos (\theta))=2 r^{d} \cos (d \theta) .
$$


$T_{d, r}(z)$ can be expanded as

$$
T_{d, r}(z)=z^{d}+\sum_{k=1}^{\lfloor d / 2\rfloor}(-1)^{k} \frac{d}{k}\left(\begin{array}{c}
d-k-1 \\
k-1
\end{array}\right) r^{2 k} z^{d-2 k}
$$

(see [10]). The graph of $T_{d, r}(z)$ oscillates $d$ times between $\pm 2 r^{d}$ on $[-2 r, 2 r]$, and $T_{d, r}(z)$ takes each value between $\pm 2 r^{d}$ exactly $d$ times on $[-2 r, 2 r]$ (counting multiplicities), so that

$$
[-2 r, 2 r]=\left\{z \in \mathbb{C}: T_{d, r}(z) \in\left[-2 r^{n}, 2 r^{n}\right]\right\} .
$$

Based on (6.2) one might hope that for a general set $E_{v}$, the polynomial $T_{d}\left(z ; E_{v}\right)$ oscillates $d$ times between $\pm 2 R_{v}^{d}$ on $E_{v}$, so that

$$
E_{v}=\left\{z \in \mathbb{C}: T_{d}\left(z ; E_{v}\right) \in\left[-2 R_{v}^{d}, 2 R_{v}^{d}\right]\right\} .
$$

Unfortunately, in general (6.3) is false: it fails, for example, if $T_{d}\left(z ; E_{v}\right)$ has a zero in one of the gaps $\left(b_{i}, a_{i+1}\right)$. However, a result of Raphael Robinson $([10$, p. 422$])$ asserts that after enlarging the intervals $\left[a_{i}, b_{i}\right]$ slightly within $U_{v}$ and choosing $d$ appropriately, we can assume that $T_{d}\left(z ; E_{v}\right)$ and $E_{v}$ do satisfy (6.3). Robinson proved this by carefully analyzing the properties of certain Schwarz-Christoffel mappings. Robinson's theorem is the key to our construction, and the absence of a purely potential-theoretic proof of it is the chief obstruction to extending the Fekete-Szegö theorem with splitting conditions to a more general setting.

In general, if $f(z) \in \mathbb{R}[z]$ is a polynomial of degree $d$, then for any $R>0$ we will say that " $f(z)$ oscillates $d$ times between $\pm R$ on a set $E \subset \mathbb{R}$ " if $\{z \in \mathbb{C}: f(z) \in[-R, R]\}=E$, and we will say that " $f(z)$ oscillates $d$ times with magnitude at least $R$ on $E$ " if $\{z \in \mathbb{C}: f(z) \in[-R, R]\} \subseteq E$.

In the following, we will assume that $E_{v}$ and $d$ have been chosen so that (6.3) holds. Since our reductions have only enlarged $E_{v}$, the hypothesis $0<h_{v}<r_{v}<\gamma\left(E_{v}\right)$ continues to hold. Put

$$
g(z)=T_{d}\left(z ; E_{v}\right), \quad n_{v}=d=\operatorname{deg}(g(z)),
$$

so that $g(z) \in \mathbb{R}[z]$ is monic of degree $n_{v}$ and

$$
E_{v}=g^{-1}\left(\left[-2 R_{v}^{n_{v}}, 2 R_{v}^{n_{v}}\right]\right)
$$

An important consequence of (6.2) and (6.4) is that by composing $g(z)$ with the polynomials $T_{m, R_{v} n_{v}}(z)$, we can obtain Chebyshev polynomials for $E_{v}$ of higher degree:

$$
T_{m, n_{v}}\left(z ; E_{v}\right)=T_{m, R_{v}^{n v}}(g(z)) .
$$

This polynomial $g(z)$ and its degree $n_{v}$ will be fixed for the rest of the construction. The constant in the proposition will be

$$
M_{v}=\max _{0 \leq h<n_{v}}\left(\left\|z^{h}\right\|_{U_{v}} / R_{v}^{h}\right) .
$$


Construction of the initial patching polynomials. The condition imposed by the place $v$ on the globally chosen constant $L$ will be that

$$
\left(\frac{h_{v}}{r_{v}}\right)^{L} \frac{M_{v}}{1-h_{v} / r_{v}}<\frac{1}{16} .
$$

Clearly this holds for all sufficiently large $L$. Assuming $L$ has been fixed, choose an integer $N_{v}$ large enough that

$$
n_{v} N_{v}>L \text { and } R_{v}^{n_{v} N_{v}}>2 r_{v}^{n_{v} N_{v}}
$$

and put

$$
\widetilde{g}(z)=T_{N_{v}, R_{v}^{n_{v}}}(g(z)) .
$$

Then $\widetilde{g}(z)$ is monic of degree $n_{v} N_{v}$, and by (6.4)

$$
E_{v}=\widetilde{g}^{-1}\left(\left[-2 R_{v}^{n_{v} N_{v}}, 2 R_{v}^{n_{v} N_{v}}\right]\right) .
$$

By continuity, there is a number $\delta>0$ such that for each monic $\widehat{g}(z)$ in $\mathbb{R}[z]$ of degree $n_{v} N_{v}$, whose coefficients differ from those of $\widetilde{g}(z)$ by at most $\delta$ in magnitude, we have

$$
|\widehat{g}(z)-\widetilde{g}(z)|<R_{v}^{n_{v} N_{v}}
$$

for all $z \in E_{v}$. Put

$$
\widehat{R}_{v}=2^{-1 /\left(n_{v} N_{v}\right)} R_{v}
$$

noting that by (6.7),

$$
r_{v}<\widehat{R}_{v}<R_{v} .
$$

Finally, choose an interval $E_{v, 0}=[c, d]$ contained in $U_{v} \backslash E_{v}$. For each integer $s$ in the range $0 \leq s<n_{v} N_{v}$, let $f_{s}(z)$ be the Chebyshev polynomial of degree $s$ for $E_{v, 0}$. Put

$$
\varrho:=\gamma\left(E_{v, 0}\right)=(d-c) / 4,
$$

so that $f_{s}(z)$ oscillates $s$ times between $\pm 2 \varrho^{s}$ on $E_{v, 0}$, and

$$
\left|f_{s}(z)\right|>2 \varrho^{s} \text { on } \mathbb{R} \backslash E_{v, 0} .
$$

In the following, we will write $\widehat{T}_{m}(z)$ for $T_{m, \widehat{R}_{v}^{n_{v} N_{v}}}(z)$. Given $n$, to define the initial polynomial $u_{v}^{(0)}(z)$, decompose $n=s+m \cdot n_{v} N_{v}$, where $s, m$ are integers and $0 \leq s<n_{v} N_{v}$, and put

$$
u_{v}^{(0)}(z)=f_{s}(z) \widehat{T}_{m}(\widetilde{g}(z)) .
$$

For the rest of the construction, we hold $s$ fixed, and view $n$ as a function of $m$. Since there are only finitely many progressions $n=s+m \cdot n_{v} N_{v}$, it suffices to prove the proposition for each $s$, and all sufficiently large $m$.

Phase 1. Patching the high-order coefficients $(k \leq L)$. Just as in the case $K_{v} \cong \mathbb{C}$, we patch the high-order coefficients of $u_{v}^{(0)}(z)$ by modifying the coefficients of $\widetilde{g}(z)$, taking advantage of "magnification". Let $B_{v}>0$ be the number specified in the proposition. 
Assume $m$ is large enough that $m>L$ and $m \delta>B_{v}$. Write $\widehat{g}^{(0)}(z)=$ $\widetilde{g}(z)$, so that $u_{v}^{(0)}(z)=f_{s}(z) \widehat{T}_{m}\left(\widehat{g}^{(0)}(z)\right)$. For each $k=1, \ldots, L$, we will define $\widehat{g}^{(k)}(z)$ by adding a number $\delta_{k} \in \mathbb{R}$ with $\left|\delta_{k}\right|<\delta$ to the $k$ th coefficient of $\widehat{g}^{(k-1)}(z)$, and patch $u^{(k-1)}(z)$ by taking $u^{(k)}(z)=f_{s}(z) \widehat{T}_{m}\left(\widehat{g}^{(k)}(z)\right)$. The choice of $\delta_{k}$ will be explained below. Since $\left|\delta_{k}\right|<\delta$, each $\widehat{g}^{(k)}(z)$ satisfies (6.9). Write

$$
f_{s}(z)=z^{s}+\sum_{h=1}^{s} a_{h} z^{s-h}, \quad \widehat{g}^{(k-1)}(z)=z^{n_{v} N_{v}}+\sum_{j=1}^{n_{v} N_{v}} b_{j} z^{n_{v} N_{v}-j}
$$

and expand

$$
\begin{aligned}
u_{v}^{(k-1)}(z)= & f_{s}(z) \widehat{T}_{m}\left(\widehat{g}^{(k-1)}(z)\right) \\
= & f_{s}(z) \widehat{g}^{(k-1)}(z)^{m} \\
& +\sum_{j=1}^{\lfloor m / 2\rfloor}(-1)^{j} \frac{m}{j}\left(\begin{array}{c}
m-j-1 \\
j-1
\end{array}\right) \widehat{R}_{v}^{2 j} f_{s}(z)\left(\widehat{g}^{(k-1)}(z)\right)^{m-2 j} \\
= & z^{n}+\sum_{l=1}^{n} c_{v, l} z^{n-l} .
\end{aligned}
$$

Each term $f_{s}(z) \widehat{g}^{(k-1)}(z)^{m-2 j}$ in the sum has degree $s+(m-2 j) n_{v} N_{v}<$ $n-L$, so the $L$ high-order coefficients of $u_{v}^{(k-1)}(z)$ are the same as those of $f_{s}(z) \widehat{g}^{(k-1)}(z)^{m}$.

Thus, just as when $K_{v} \cong \mathbb{C}$ (see (5.10)), the $k$ th coefficient $c_{v, k}$ is a polynomial in $m$ and $b_{1}, \ldots, b_{k}$ of the form

$$
c_{v, k}=m b_{k}+Q_{s, k}\left(m, b_{1}, \ldots, b_{k-1}\right),
$$

which depends linearly on $b_{k}$. If $\delta_{k}$ is added to the coefficient $b_{k}$ in $\widehat{g}^{(k-1)}(z)$, then $c_{v, k}$ is changed to $c_{v, k}+m \delta_{k}$ while the coefficients of $u_{v}^{(k-1)}(z)$ with $j<k$ remain unchanged. Hence, since $m \delta>B_{v}$, we can sequentially vary the first $L$ coefficients of $u_{v}^{(0)}(z)$ by quantities $\Delta_{v, k}$ up to $B_{v}$ in magnitude, by sequentially varying the first $L$ coefficients of $\widetilde{g}(z)$ by quantities $\delta_{k}=\Delta_{v, k} / m$ at most $\delta$ in magnitude.

The choice of the numbers $\Delta_{v, k}$ will be made on the basis of global considerations; for the remainder of this section we assume that $\Delta_{v, 1}, \ldots, \Delta_{v, L} \in \mathbb{R}$ have been fixed, and write $\widehat{g}(z)$ for $\widehat{g}^{(L)}(z)$. In the discussion below, we use only the fact that $\widehat{g}(z) \in \mathbb{R}[z]$ satisfies (6.9) and that it is monic with degree $n_{v} N_{v}>L$.

Phase 2. Patching the middle coefficients $(L<k \leq n-s)$. By (6.8), $\widetilde{g}(z)$ oscillates $n_{v} N_{v}$ times between $\pm 2 R_{v}^{n_{v} N_{v}}$ on $E_{v}$. At any point $z$ where $|\widetilde{g}(z)|=2 R_{v}^{n_{v} N_{v}}$, it follows from (6.9) that $|\widehat{g}(z)|>R_{v}^{n_{v} N_{v}}$, so $\widehat{g}(z)$ oscillates 
$n_{v} N_{v}$ times with magnitude at least $R_{v}^{n_{v} N_{v}}=2 \widehat{R}_{v}^{n_{v} N_{v}}$ on $E_{v}$. The set

$$
\widehat{E}_{v}:=\left\{z \in \mathbb{C}: \widehat{g}(z) \in\left[-2 \widehat{R}_{v}^{n_{v} N_{v}}, 2 \widehat{R}_{v}^{n_{v} N_{v}}\right]\right\}
$$

satisfies $\widehat{E}_{v} \subset E_{v}$, and $\widehat{g}(z)$ oscillates $n_{v} N_{v}$ times between $\pm 2 \widehat{R}_{v}^{n_{v} N_{v}}$ on $\widehat{E}_{v}$. We have

$$
u_{v}^{(L)}(z)=f_{s}(z) \widehat{T}_{m}(\widehat{g}(z)) \text { and } n=s+m \cdot n_{v} N_{v}=\operatorname{deg}\left(u_{v}^{(L)}(z)\right) .
$$

Since $\widehat{T}_{m}(z):=T_{m, \widehat{R}_{v}^{n_{v} N_{v}}}(z)$ is the Chebyshev polynomial of degree $m$ for $\left[-2 \widehat{R}_{v}^{n_{v} N_{v}}, 2 \widehat{R}_{v}^{n_{v} N_{v}}\right]$, it follows that $\widehat{T}_{m}(\widehat{g}(z))$ oscillates $n_{v} N_{v} m$ times between $\pm 2 \widehat{R}_{v}^{n_{v} N_{v} m}$ on $\widehat{E}_{v}$.

Our goal is to patch $u_{v}^{(L)}(z)$ in such a way that $u_{v}^{(n)}(z)$ oscillates with large magnitude on $\widehat{E}_{v}$. Write $T_{h}(z)=T_{h, R_{v}^{n v}}(z)$ for the Chebyshev polynomial of the interval $\left[-2 R_{v}^{n_{v}}, 2 R_{v}^{n_{v}}\right]$, and let $w_{v}^{(k)}(z)$ be the monic polynomial of degree $n-k-s$ given by

$$
w_{v}^{(k)}(z)=z^{h_{1}} T_{h_{2}}(g(z)) \widehat{T}_{h_{3}}(\widehat{g}(z))
$$

where the integers $h_{1}, h_{2}, h_{3}$ are determined by the conditions

$$
\begin{gathered}
n-k-s=h_{1}+h_{2} n_{v}+h_{3} n_{v} N_{v}, \\
0 \leq h_{1}<n_{v}, \quad 0 \leq h_{2}<N_{v}, \quad 0 \leq h_{3}<m .
\end{gathered}
$$

At the $k$ th step of the patching process, $L<k \leq n-s$, we will put

$$
u_{v}^{(k)}(z)=u_{v}^{(k-1)}(z)+\Delta_{v, k} f_{s}(z) w_{v, k}(z),
$$

where $\Delta_{v, k} \in \mathbb{R}$ satisfies $\left|\Delta_{v, k}\right| \leq h_{v}^{k}$.

We now seek to bound $\left|w_{v, k}(z)\right|$ on $\widehat{E}_{v}$. From (6.5), together with (6.10) and $0 \leq h_{1}<n_{v}$, it follows that

$$
\left\|z^{h_{1}}\right\|_{\widehat{E}_{v}} \leq M_{v} R_{v}^{h_{1}} \leq M_{v} \cdot 2 \widehat{R}_{v}^{h_{1}} .
$$

By the properties of Chebyshev polynomials and the fact that $h_{2}<N_{v}$,

$$
\left\|T_{h_{2}}(g(z))\right\|_{\widehat{E}_{v}} \leq\left\|T_{h_{2}}(g(z))\right\|_{E_{v}}=2 R_{v}^{h_{2} n_{v}} \leq 2 \cdot 2 \widehat{R}_{v}^{h_{2} n_{v}} .
$$

Likewise, by the properties of Chebyshev polynomials,

Thus,

$$
\left\|\widehat{T}_{h_{3}}(\widehat{g}(z))\right\|_{\widehat{E}_{v}}=2 \widehat{R}_{v}^{h_{3} n_{v} N_{v}} .
$$

$$
\left\|w_{v, k}(z)\right\|_{\widehat{E}_{v}} \leq 16 M_{v} \widehat{R}_{v}^{n-k}
$$

At the end of this phase of the patching process, we will have

$$
\begin{aligned}
u_{v}^{(n-s)}(z) & =u_{v}^{(L)}(z)+\sum_{k=L+1}^{n-s} \Delta_{v, k} f_{s}(z) w_{v}^{(k)}(z) \\
& =f_{s}(z)\left(\widehat{T}_{m}(\widehat{g}(z))+\sum_{k=L+1}^{n-s} \Delta_{v, k} w_{v}^{(k)}(z)\right) .
\end{aligned}
$$


Put $q(z)=\widehat{T}_{m}(\widehat{g}(z))+\sum_{k=L+1}^{n-s} \Delta_{v, k} w_{v}^{(k)}(z)$. By the inequalities above, and (6.6), for $z \in \widehat{E}_{v}$,

$$
\begin{aligned}
\left|q(z)-\widehat{T}_{m}(\widehat{g}(z))\right| & \leq 16 M_{v} \sum_{k=L+1}^{n} h_{v}^{k} \widehat{R}_{v}^{n-k} \\
& \leq 16 M_{v} \sum_{k=L}^{\infty}\left(\frac{h_{v}}{r_{v}}\right)^{k} \widehat{R}_{v}^{n} \leq \widehat{R}_{v}^{n} .
\end{aligned}
$$

Since $\widehat{T}_{m}(\widehat{g}(z))$ oscillates $n_{v} N_{v} m$ times between $\pm 2 \widehat{R}_{v}^{n_{v} N_{v} m}$ on $\widehat{E}_{v}$, it follows that $q(z)$ oscillates $n_{v} N_{v} m$ times with magnitude at least $\widehat{R}_{v}^{n_{v} N_{v} m}$ on $\widehat{E}_{v}$, and also that

$$
\|q(z)\|_{\widehat{E}_{v}} \leq 3 \widehat{R}_{v}^{n_{v} N_{v} m} .
$$

Before proceeding further, we will need a lemma.

LEMma 6.2. Let $E \subset \mathbb{R}$ be a compact set of positive capacity with connected complement, and put $R=\gamma(E)$. Then for any compact set $F \subset \mathbb{C} \backslash E$ there is a constant $C(E, F)$ with the following property: if $f(z)$ is a monic polynomial of degree $d$ with all its roots in $E$, and if $\|f\|_{E} \leq B R^{d}$ for some number $B$, then for all $z \in F$,

$$
\left|G(z, \infty ; E)+\log (R)-\frac{1}{d} \log (|f(z)|)\right| \leq \frac{C(E, F) \log (B)}{d} .
$$

Proof. Let $B_{0}$ be such that $\|f\|_{E}=B_{0} R^{d}$, and put

$$
h(z)=G(z, \infty ; E)+\frac{1}{d} \log \left(B_{0}\right)+\log (R)-\frac{1}{d} \log (|f(z)|) .
$$

Then $h(z)$ is harmonic in $\mathbb{C} \backslash E$, and $\lim _{z \rightarrow \infty} h(z)=\frac{1}{d} \log \left(B_{0}\right)$, so $h(z)$ extends to a function harmonic in $\mathbb{P}^{1}(\mathbb{C}) \backslash E$ with $h(\infty)=\frac{1}{d} \log \left(B_{0}\right)$. For each $x \in \partial\left(\mathbb{P}^{1}(\mathbb{C}) \backslash E\right)$,

$$
\liminf _{\substack{z \rightarrow x \\ z \in \mathbb{P}^{1}(\mathbb{C}) \backslash E}} h(z) \geq 0 .
$$

By the minimum principle for harmonic functions, we have $h(z) \geq 0$ for all $z \in \mathbb{P}^{1}(\mathbb{C}) \backslash E$. Hence, by Harnack's theorem, there is a constant $C_{0}$ such that for all $z \in F$,

$$
0 \leq h(z) \leq C_{0} h(\infty)=C_{0} \frac{1}{d} \log \left(B_{0}\right) .
$$

Thus, we can take $C(E, F)=\max \left(1, C_{0}-1\right)$.

Phase 3. Patching the low-order coefficients $(n-s<k \leq n)$. To motivate our procedure for patching the low order coefficients, we need lower bounds for $\left|f_{s}(z)\right|$ on $\widehat{E}_{v}$ and for $|q(z)|$ on $E_{v, 0}$.

Since $f_{s}(z)$ is the Chebyshev polynomial for $E_{v, 0}$, and $\widehat{E}_{v} \subset E_{v} \subset \mathbb{R}$ is disjoint from $E_{v, 0}$, it follows from (6.12) that $\left|f_{s}(z)\right| \geq 2 \varrho^{s}$ on $\widehat{E}_{v}$. Thus, at 
each point of $\widehat{E}_{v}$ where $|q(z)| \geq \widehat{R}_{v}^{n_{v} N_{v} m}$, we have

$$
\left|u_{v}^{(n-s)}(z)\right|=\left|f_{s}(z) q(z)\right| \geq 2 \varrho^{s} \widehat{R}_{v}^{n_{v} N_{v} m}=2\left(\varrho / \widehat{R}_{v}\right)^{s} \widehat{R}_{v}^{n} .
$$

On the other hand, there is a positive lower bound $\sigma$ for $G\left(z, \infty ; \widehat{E}_{v}\right)$ on $E_{v, 0}$. Applying Lemma 6.2, with $E=\widehat{E}_{v}$ and $F=E_{v, 0}$, taking $f(z)=q(z)$ and using (6.16), we see that if $m$ is sufficiently large, then for all $z \in E_{v, 0}$,

$$
\frac{1}{n_{v} N_{v} m} \log (|q(z)|) \geq \sigma+\log \left(\widehat{R}_{v}\right)-\frac{C\left(\widehat{E}_{v} ; E_{v, 0}\right) \log (3)}{n_{v} N_{v} m} \geq \log \left(\widehat{R}_{v}\right),
$$

and so at each point of $E_{v, 0}$ where $\left|f_{s}(z)\right|=2 \varrho^{s}$ we also have

$$
\left|u_{v}^{(n-s)}(z)\right|=\left|f_{s}(z) q(z)\right| \geq 2 \varrho^{s} \widehat{R}_{v}^{n_{v} N_{v} m}=2\left(\varrho / \widehat{R}_{v}\right)^{s} \widehat{R}_{v}^{n} .
$$

Put

$$
A_{1}=\min _{0 \leq s<n_{v} N_{v}}\left(2\left(\varrho / \widehat{R}_{v}\right)^{s}\right), \quad A_{2}=\max _{0 \leq s<n_{v} N_{v}}\left(\left\|z^{s}\right\|_{U_{v}} / h_{v}^{s}\right) .
$$

Since $\widehat{R}_{v}=2^{-1 /\left(n_{v} N_{v}\right)} R_{v}$, these constants depend only on $E_{v}, U_{v}$, and our choice of $n_{v}$ and $N_{v}$. As $f_{s}(z)$ has all its zeros in $E_{v, 0}$, it has constant sign on each subinterval of $\widehat{E}_{v}$. Similarly, since $q(z)$ has all its zeros in $\widehat{E}_{v}$, it has constant sign on $E_{v, 0}$. Since $q(z)$ oscillates $n_{v} N_{v} m$ times with magnitude at least $\widehat{R}_{v}^{n_{v} N_{v} m}$ on $\widehat{E}_{v}$, and $f_{s}(z)$ oscillates $s$ times between $\pm 2 \varrho^{s}$ on $E_{v, 0}$, it follows that $u_{v}^{(n-s)}(z)$ oscillates $n$ times, with magnitude at least $A_{1} \widehat{R}_{v}^{n}$, on $\widehat{E}_{v} \cup E_{v, 0}$.

To patch the coefficients $c_{v, k}$ for $n-s<k \leq n$, set

$$
u_{v}^{(k)}(z)=u_{v}^{(k-1)}(z)+\Delta_{v, k} z^{n-k} .
$$

On the set $\widehat{E}_{v} \cup E_{v, 0}$, we have $\left|\Delta_{v, k} z^{n-k}\right| \leq h_{v}^{k} A_{2} h_{v}^{n-k}=A_{2} h_{v}^{n}$, and the total patching correction in passing from $u_{v}^{(n-s)}(z)$ to $u_{v}^{(n)}(z)$ has magnitude at most $n_{v} N_{v} \cdot A_{2} h_{v}^{n}$. Recall that by $(6.11), h_{v}<r_{v}<\widehat{R}_{v}$. If $m$ (hence $n$ ) is sufficiently large then we will have

$$
n_{v} N_{v} A_{2} h_{v}^{n} \leq \frac{1}{2} A_{1} \widehat{R}_{v}^{n}
$$

Consequently, if $n$ is large enough that (6.17) and (6.18) hold, then $u_{v}^{(n)}(z)$ oscillates $n$ times, with magnitude at least $\frac{1}{2} A_{1} \widehat{R}_{v}^{n}$, on $\widehat{E}_{v} \cup E_{v, 0}$. It follows that $u_{v}^{(n)}(z)$ has all its roots in $\widehat{E}_{v} \cup E_{v, 0}$, and hence in $U_{v}$.

7. Local patching for nonarchimedean PL-domains. When $K_{v}$ is nonarchimedean, and $E_{v}$ is a PL-domain, our patching procedure generalizes a method introduced by Cantor [3]. 
Proposition 7.1. Let $K_{v}$ be nonarchimedean, and suppose

$$
E_{v}=\bigcup_{i=1}^{M} B\left(a_{i}, r_{i}\right)
$$

is a finite union of balls with radii in the value group of $\mathbb{C}_{v}^{\times}$, stable under $\mathrm{Gal}_{\mathrm{c}}\left(\mathbb{C}_{v} / K_{v}\right)$, and such that $E_{v} \cap K_{v}$ is nonempty. There is a constant $M_{v}$, depending only on $E_{v}$, with the following property. Let $0<h_{v}<r_{v}<\gamma\left(E_{v}\right)$ be given. For each integer $L$ large enough that

$$
\left(\frac{h_{v}}{r_{v}}\right)^{L} M_{v} \leq \frac{1}{2}
$$

there is a constant $B_{v}$ (depending on $L$ ), such that for each sufficiently large $n$ there is a monic polynomial $u_{v}^{(0)}(z) \in K_{v}[z]$ of degree $n$ whose roots belong to $E_{v}$, and which can be patched with any numbers $\Delta_{v}^{(k)} \in K_{v}$ satisfying

$$
\left|\Delta_{v}^{(k)}\right|_{v} \leq \begin{cases}B_{v} & \text { for } k \leq L, \\ h_{v}^{k} & \text { for } k>L,\end{cases}
$$

in such a way that its roots remain in $E_{v}$.

Proof. We begin with some reductions. In the decomposition $E_{v}=$ $\bigcup_{i=1}^{M} B\left(a_{i}, r_{i}\right)$, we can assume that the balls $B\left(a_{i}, r_{i}\right)$ are pairwise disjoint and that the $a_{i}$ belong to $\widetilde{K}_{v}$. Furthermore, since by hypothesis $E_{v} \cap K_{v}$ is nonempty, some $B\left(a_{i}, r_{i}\right)$ contains a point of $K_{v}$; we can assume that $i=1$ and that $a_{1} \in K_{v}$. Lemma 12.1 of Appendix II shows there is a polynomial $g_{0}(z) \in \widetilde{K}_{v}[z]$ of the form $g_{0}(z)=c_{0} \prod_{i=1}^{M}\left(z-a_{i}\right)^{d_{i}}$, with each $d_{i}>0$, such that

$$
E_{v}=\left\{z \in \mathbb{C}_{v}:\left|g_{0}(z)\right|_{v} \leq 1\right\} .
$$

If $\sigma \in \operatorname{Gal}_{c}\left(\mathbb{C}_{v} / K_{v}\right)$, then since $E_{v}$ is Galois-stable,

$$
\left|g_{0}(z)\right|_{v} \leq 1 \Leftrightarrow z \in E_{v} \Leftrightarrow\left|\left(\sigma g_{0}\right)(z)\right|_{v} \leq 1 .
$$

Thus taking the product of the conjugates of $g_{0}(z)$ over $K_{v}$, and scaling the product so it is monic, we obtain a polynomial $g(z) \in K_{v}[z]$ of degree $n_{v}$, say, and a number $R_{v}$ in the value group of $\mathbb{C}_{v}^{\times}$for which

$$
E_{v}=\left\{z \in \mathbb{C}_{v}:|g(z)|_{v} \leq R_{v}^{n_{v}}\right\} .
$$

By (3.4), $R_{v}=\gamma\left(E_{v}\right)$. The polynomial $g(z)$ will be fixed for the rest of the construction.

We will need the nonarchimedean Maximum Modulus Principle in the following form (see [3, Theorem 3.1.1, p. 180] or [11, Theorem 1.4.2, p. 51]).

Lemma 7.2 (Maximum Principle with Distinguished Boundary). Let $f(z)$ $\in \mathbb{C}_{v}(z)$ be a nonconstant rational function, and let $R$ belong to the value 
group of $\mathbb{C}_{v}^{\times}$. Put

$$
\begin{aligned}
D & =\left\{z \in \mathbb{P}^{1}\left(\mathbb{C}_{v}\right):|f(z)|_{v} \leq R\right\}, \\
\partial D & =\partial D(f)=\left\{z \in \mathbb{P}^{1}\left(\mathbb{C}_{v}\right):|f(z)|_{v}=R\right\} .
\end{aligned}
$$

Then any rational function $F(z) \in \mathbb{C}_{v}(z)$ with no poles in $D$ achieves its maximum absolute value on $D$ at a point of $\partial D$. In particular, for any $M$, if $|F(z)|_{v} \leq M$ on $\partial D$, then $|F(z)|_{v} \leq M$ on all of $D$.

Put

$$
\begin{aligned}
D_{v} & =\left\{z \in \mathbb{P}^{1}\left(\mathbb{C}_{v}\right):|g(z)|_{v} \geq R_{v}^{n_{v}}\right\} \\
& =\left\{z \in \mathbb{P}^{1}\left(\mathbb{C}_{v}\right):|1 / g(z)|_{v} \leq 1 / R_{v}^{n_{v}}\right\}
\end{aligned}
$$

noting that

$$
\partial E_{v}(g)=\partial D_{v}(1 / g)=\left\{z \in \mathbb{C}_{v}:|g(z)|_{v}=R_{v}^{n_{v}}\right\} .
$$

We will write $\partial D_{v}=\partial E_{v}$ for this common boundary. Write $a=a_{1}$, and note that $a$ is a root of $g(z)$. By the maximum modulus principle, applied to $F(z)=1 /(z-a)$ on $D_{v}$ relative to $\partial D_{v}$, the number $\min _{z \in \partial E_{v}}\left(|z-a|_{v}\right)$ exists and is positive. Put

$$
\begin{aligned}
& C_{v}^{-}=\min _{0 \leq h<n_{v}}\left(\left(\min _{z \in \partial E_{v}}\left|(z-a)^{h}\right|_{v}\right) / R_{v}^{h}\right), \\
& C_{v}^{+}=\max _{0 \leq h<n_{v}}\left(\left(\max _{z \in \partial E_{v}}\left|(z-a)^{h}\right|_{v}\right) / R_{v}^{h}\right) .
\end{aligned}
$$

The constant $M_{v}$ in the proposition will be

$$
M_{v}=C_{v}^{+} / C_{v}^{-} \text {. }
$$

The condition imposed on the globally chosen constant $L$ by the place $v$ is

$$
M_{v}\left(\frac{h_{v}}{r_{v}}\right)^{L} \leq \frac{1}{2},
$$

and the constant $B_{v}$ in the proposition will be

$$
B_{v}=\frac{1}{2} \min _{1 \leq k \leq L}\left(R_{v}^{k} / M_{v}\right) .
$$

The patching construction. If $n=s+m n_{v}$, where $s, m$ are integers with $0 \leq s<n_{v}$, we take the initial patching polynomial to be

$$
u_{v}^{(0)}(z)=(z-a)^{s} g(z)^{m} \in K_{v}[z]
$$

By (7.5) and (7.6), for all $z \in \partial E_{v}$,

$$
\left|u_{v}^{(0)}(z)\right|_{v} \geq C_{v}^{-} R_{v}^{n}
$$

The Maximum Modulus Principle, applied to $1 / u_{v}^{(0)}(z)$ on $\partial D_{v}$, shows this holds for all $z \notin E_{v}$.

Let $w_{v}^{(k)}(z)$ be the monic polynomial of degree $n-k$

$$
w_{v}^{(k)}(z)=(z-a)^{h_{1}} g(z)^{h_{2}}
$$


where the integers $h_{1}, h_{2}$, are determined by

$$
n-k=h_{1}+h_{2} n_{v}, \quad 0 \leq h_{1}<n_{v}, 0 \leq h_{2}<m .
$$

By (7.8) and (7.3), for all $z \in E_{v}$,

$$
\left|w_{v}^{(k)}(z)\right|_{v} \leq C_{v}^{+} R_{v}^{h_{1}} R_{v}^{h_{2} n_{v}}=C_{v}^{+} R_{v}^{n-k} .
$$

At the $k$ th step of the patching process, $1 \leq k \leq n$, we will put

$$
u_{v}^{(k)}(z)=u_{v}^{(k-1)}(z)+\Delta_{v, k} w_{v, k}(z)
$$

where $\Delta_{v, k} \in K_{v}$ is chosen on the basis of global considerations, and satisfies the conditions in the proposition. At the end of the construction,

$$
u_{v}^{(n)}(z)=u_{v}^{(0)}(z)+\sum_{k=1}^{n} \Delta_{v}^{(k)} w_{v}^{(k)}(z) .
$$

We claim that the conditions on $\left|\Delta_{v}^{(k)}\right|_{v}$ imply that for all $z \in E_{v}$,

$$
\left|\sum_{k=1}^{n} \Delta_{v}^{(k)} w_{v}^{(k)}(z)\right|_{v} \leq \frac{1}{2} C_{v}^{-} R_{v}^{n} .
$$

By the ultrametric inequality, it suffices to check this for each $k$. When $k \leq L$ we have $\left|\Delta_{v}^{(k)}\right|_{v} \leq B_{v} \leq \frac{1}{2} R_{v}^{k} / M_{v}$ by (7.10), while if $k>L$, then $\left|\Delta_{v}^{(k)}\right|_{v} \leq h_{v}^{k} \leq \frac{1}{2} r_{v}^{k} / M_{v} \leq \frac{1}{2} R_{v}^{k} / M_{v}$ by (7.9). Thus, in either case, since $M_{v}=C_{v}^{+} / C_{v}^{-}$, by $(7.14)$,

$$
\left|\Delta_{v}^{(k)} w_{v}^{(k)}(z)\right|_{v} \leq\left(\frac{1}{2} R_{v}^{k} / M_{v}\right)\left(C_{v}^{+} R_{v}^{n-k}\right)=\frac{1}{2} C_{v}^{-} R_{v}^{n} .
$$

We now apply the Maximum Modulus Principle to

$$
F(z)=\left(u_{v}^{(n)}(z)-u_{v}^{(0)}(z)\right) / u_{v}^{(0)}(z)=\left(\sum_{k=1}^{n} \Delta_{v}^{(k)} w_{v}^{(k)}(z)\right) / u_{v}^{(0)}(z)
$$

on the domain $D_{v}$, relative to the boundary $\partial D_{v}=\partial E_{v}$.

Here $u_{v}^{(n)}(z)$ and $u_{v}^{(0)}(z)$ are monic polynomials of degree $n$, and the zeros of $u_{v}^{(0)}(z)=(z-a)^{s} g(z)^{m}$ belong to $\mathbb{P}^{1}\left(\mathbb{C}_{v}\right) \backslash D_{v}$, so $F(z)$ has no poles in $D_{v}$. On $\partial D_{v}=\partial E_{v},(7.16)$ and (7.11) show that

$$
|F(z)|_{v} \leq 1 / 2 \text {. }
$$

Hence $|F(z)|_{v} \leq 1 / 2$ throughout $D_{v}$. This means that for all $z \notin E_{v}$,

$$
\left|u_{v}^{(n)}(z)-u_{v}^{(0)}(z)\right|_{v} \leq \frac{1}{2}\left|u_{v}^{(0)}(z)\right|_{v}
$$

and so by the ultrametric inequality, for all $z \notin E_{v}$,

$$
\left|u_{v}^{(n)}(z)\right|_{v}=\left|u_{v}^{(0)}(z)\right|_{v} \geq C_{v}^{-} R_{v}^{n}>0 .
$$

In particular, $u_{v}^{(n)}(z)$ has all its roots in $E_{v}$. 
8. Local patching for nonarchimedean compact sets. Let $K_{v}$ be nonarchimedean, and suppose $E_{v} \subset K_{v}$ is compact. By our preliminary reductions, we can assume $E_{v}$ is a finite disjoint union of cosets of $O_{v}$,

$$
E_{v}=\bigcup_{i=1}^{M}\left(a_{i}+b_{i} O_{v}\right),
$$

where the $a_{i}, b_{i} \in K_{v}$ and the $b_{i} \neq 0$. Let $R$ be such that $E_{v} \subset B(0, R)$.

Proposition 8.1. Suppose $K_{v}$ is nonarchimedean and

$$
E_{v}=\bigcup_{i=1}^{M}\left(a_{i}+b_{i} O_{v}\right) \subset K_{v} .
$$

There is a number $M_{v}$, depending only on $E_{v}$, with the following property. Let $0<h_{v}<r_{v}<\gamma\left(E_{v}\right)$ be given. If $L \geq 1$ is large enough that

$$
L\left(\frac{h_{v}}{r_{v}}\right)^{L} M_{v} \leq 1,
$$

then there is a constant $B_{v}$ (depending on $L$ ) such that for each sufficiently large $n$, there is a monic polynomial $u_{v}^{(0)}(z) \in K_{v}[z]$ of degree $n$, which has all its roots in $E_{v}$, and can be patched with arbitrary $\Delta_{v}^{(k)} \in K_{v}$ satisfying

$$
\left|\Delta_{v}^{(k)}\right|_{v} \leq \begin{cases}B_{v} & \text { for } k \leq L, \\ h_{v}^{k} & \text { for } k>L,\end{cases}
$$

in such a way that its roots remain in $E_{v}$.

Before giving the proof we have several tasks. First, we need to construct the "basic well-distributed sequence for $E_{v}$ " and define the notion of a "regular sequence in $E_{v}$ ". Second, we need to introduce generalized Stirling polynomials, which play a role analogous to those of Chebyshev polynomials in the case $K_{v} \cong \mathbb{R}$ (this idea goes back to Cantor [3]). Finally, we must prove several lemmas which govern the patching process.

The basic well-distributed sequence. First consider the case where $E_{v}=$ $O_{v}$. Write $q=q_{v}=\#\left(O_{v} / \pi_{v} O_{v}\right)$, and let $\beta_{v}(k)$, for $k=0, \ldots, q-1$, be a set of representatives for $O_{v} / \pi_{v} O_{v}$, with $\beta_{v}(0)=0$. For $k \geq q$, expand $k$ as

$$
k=\sum_{i=0}^{N} d_{i}(k) q^{i}
$$

where $N=\left\lfloor\log _{q}(k)\right\rfloor$ and $0 \leq d_{i}(k) \leq q-1$ are the base $q$ digits of $k$; put

$$
\beta_{v}(k)=\sum_{i=0}^{N} \beta_{v}\left(d_{i}(k)\right) \pi_{v}^{i} .
$$


In this way, the sequence $\left\{\beta_{v}(k)\right\}_{0 \leq k<\infty}$ extends $\left\{\beta_{v}(k)\right\}_{0 \leq k<q}$, and uniformly fills out the cosets of $O_{v} / \pi_{v}^{e} \bar{O}_{v}$ for each $e \geq 0$. Define val $\operatorname{val}_{q}(k)$ to be the smallest $i$ such that $d_{i}(k) \neq 0$. Then it is easy to see that for each $k$,

$$
\operatorname{ord}_{v}\left(\beta_{v}(k)\right)=\operatorname{val}_{q}(k) \text {, }
$$

and that for all $k \neq l$,

$$
\operatorname{ord}_{v}\left(\beta_{v}(k)-\beta_{v}(l)\right)=\operatorname{val}_{q}(|k-l|) .
$$

We call $\left\{\beta_{v}(k)\right\}$ the basic well-distributed sequence in $O_{v}$. Note that for each $n>0$, if $k, l<n$ and $k \neq l$, then

$$
\operatorname{ord}_{v}\left(\beta_{v}(k)-\beta_{v}(l)\right) \leq\left\lfloor\log _{v}(n)\right\rfloor,
$$

while for each $z \in O_{v}$, there is a $k<n$ such that

$$
\operatorname{ord}_{v}\left(z-\beta_{v}(k)\right) \geq\left\lfloor\log _{v}(n)\right\rfloor .
$$

Now consider an arbitrary set of the form $E_{v}=\bigcup_{i=1}^{M} E_{v, i}$, where $E_{v, i}=$ $a_{i}+b_{i} O_{v}$ with $a_{i}, b_{i} \in K_{v}, b_{i} \neq 0$, and where the cosets are disjoint. Let $\mu$ be the equilibrium distribution of $E_{v}$, and define weights

$$
w_{i}=\mu\left(E_{v, i}\right), \quad i=1, \ldots, M .
$$

Then each $w_{i}>0$ as was noted earlier, and $\sum_{i=1}^{M} w_{i}=1$. We want the basic well-distributed sequence in $E_{v}$ to assign elements to each $E_{v, i}$ in proportion to its weight $w_{i}$. That this can be done, with small error, follows from a combinatorial lemma due to Balinski and Young ([2, Theorem 3, p. 714]):

Lemma 8.2. Let $w_{1}, \ldots, w_{M}>0$ be such that $\sum_{i} w_{i}=1$. Then there is a 1-1 correspondence $\Phi: \mathbb{N} \rightarrow \mathbb{N}^{M}, \Phi(n)=\left(\Phi_{1}(n), \ldots, \Phi_{M}(n)\right)$, such that:

(A) For each $i, \Phi_{i}(0)=0$ and $\Phi_{i}(n)$ is nondecreasing with $n$;

(B) For each $n \geq 0, \sum_{i=1}^{M} \Phi_{i}(n)=n$;

(C) For each $n$ and $i,\left\lfloor w_{i} n\right\rfloor \leq \Phi_{i}(n) \leq\left\lceil w_{i} n\right\rceil$. In particular, if $w_{i} n \in \mathbb{N}$, then $\Phi_{i}(n)=w_{i} n$.

REMARK. This lemma has an unusual source. It was originally established in the context of the "Alabama Paradox" concerning the apportionment of members in the U.S. House of Representatives. The question was, is there a rule for allotting representatives to the various states in proportion to population, in such a way that no state loses representatives when the size of the House is increased? The author thanks Peter Rice for pointing the lemma out to him.

Clearly for each $n$ there is exactly one index $i$ for which $\Phi_{i}(n+1)>\Phi_{i}(n)$. We will write $i(n)$ for this $i$. 
Definition 8.1. The basic well-distributed sequence in $E_{v}$ is the sequence $\left\{\lambda_{v}(n)\right\}_{n \geq 0}$ defined by

$$
\lambda_{v}(n)=a_{i(n)}+b_{i(n)} \cdot \beta_{v}\left(\Phi_{i(n)}(n)\right) .
$$

That is, the $n$th element of the sequence is assigned to $E_{v, i(n)}$, and the elements assigned to $E_{v, i}=a_{i}+b_{i} O_{v}$ fill out $E_{v, i}$ like the basic well-distributed sequence in $O_{v}$. Among $\lambda_{v}(0), \ldots, \lambda_{v}(n-1)$, precisely $\Phi_{i}(n)$ elements belong to $E_{v, i}$. If $\lambda_{v}(k)$ and $\lambda_{v}(l)$ belong to distinct cosets $E_{v, i}, E_{v, j}$, then

$$
\left|\lambda_{v}(k)-\lambda_{v}(l)\right|_{v}=\left|a_{i}-a_{j}\right|_{v}
$$

while if they belong to the same coset $E_{v, i}$, then, writing $r_{i}=\left|b_{i}\right|_{v}$,

$$
\left|\lambda_{v}(k)-\lambda_{v}(l)\right|_{v}=r_{i}\left|\pi_{v}\right|_{v}^{\mathrm{val}_{q}\left(\Phi_{i}(k)-\Phi_{i}(l)\right)} .
$$

Note that the basic well-distributed sequence in $E_{v}$ depends on the representation of $E_{v}$ as a union of sets $a_{i}+b_{i} O_{v}$, and also on the choice of the function $\Phi$ (which in general is not unique). For the remainder of the construction we will assume these are fixed.

Stirling polynomials and regular sequences. The classical Stirling polynomial of degree $n$ is $S_{n}(z)=\prod_{i=0}^{n-1}(z-i)$. Pólya introduced Stirling polynomials for the rings of integers $O_{v}$, putting

$$
S_{n}\left(z ; O_{v}\right)=\prod_{k=0}^{n-1}\left(z-\beta_{v}(k)\right)
$$

(cf. [8]). For general $E_{v}$, we put $S_{0}\left(z ; E_{v}\right)=1$ and define the Stirling polynomial of degree $n \geq 1$ for $E_{v}$ to be

$$
S_{n}\left(z ; E_{v}\right)=\prod_{k=0}^{n-1}\left(z-\lambda_{v}(k)\right) .
$$

However, for our needs it is not enough to consider Stirling polynomials alone, but also polynomials with roots which behave sufficiently like them.

LEMma 8.3. The basic well-distributed sequence in $E_{v}$ has the following property. Put

$$
A_{0}=\max \left(0, \max _{i}\left(\operatorname{ord}_{v}\left(b_{i}\right)\right)\right) .
$$

Then for each $n>0$ and each $k, l$ with $0 \leq k, l<n$ and $k \neq l$,

$$
\operatorname{ord}_{v}\left(\lambda_{v}(k)-\lambda_{v}(l)\right)<A_{0}+\log _{v}(n) .
$$

Proof. Let $n, k$ and $l$ be as above. If $i(k) \neq i(l)$, then $\operatorname{ord}_{v}\left(a_{i(k)}-a_{i(l)}\right)<$ $\operatorname{ord}_{v}\left(b_{i(k)}\right) \leq A_{0}$, so

$$
\operatorname{ord}_{v}\left(\lambda_{v}(k)-\lambda_{v}(l)\right)=\operatorname{ord}_{v}\left(a_{i(k)}-a_{i(l)}\right)<A_{0}+\log _{v}(n) .
$$

On the other hand, if $i(k)=i(l)=i$, then

$$
\operatorname{ord}_{v}\left(\lambda_{v}(k)-\lambda_{v}(l)\right)=\operatorname{ord}_{v}\left(b_{i}\right)+\operatorname{val}_{q}\left(\left|\Phi_{i}(k)-\Phi_{i}(l)\right|\right)<A_{0}+\log _{v}(n) \text {. }
$$


REMARK 8.1. $A_{0}$ has the following properties:

(1) $A_{0} \geq 0$

(2) $A_{0} \geq-\log _{v}(R)$ for any $R$ with $E_{v} \subset B(0, R)$;

(3) $A_{0}>\operatorname{ord}_{v}\left(a_{i}-a_{j}\right)$ for each $i \neq j$; and

(4) if $\alpha \in K_{v}$ satisfies $\operatorname{ord}_{v}(\alpha-x) \geq A_{0}$ for some $x \in E_{v}$, then $\alpha \in E_{v}$.

Definition 8.2. A regular sequence of length $n$ in $E_{v}$ is a sequence $\left\{\alpha_{0}, \ldots, \alpha_{n-1}\right\} \subset E_{v}$ such that

$$
\operatorname{ord}_{v}\left(\alpha_{k}-\lambda_{v}(k)\right) \geq A_{0}+\log _{v}(n)
$$

for each $k=0, \ldots, n-1$.

Thus, in a regular sequence of length $n$, if $0 \leq k, l<n$ and $k \neq l$ then

$$
\operatorname{ord}_{v}\left(\alpha_{k}-\alpha_{l}\right)=\operatorname{ord}_{v}\left(\lambda_{v}(k)-\lambda_{v}(l)\right)<A_{0}+\log _{v}(n) .
$$

We now establish some simple properties of regular sequences.

LEMma 8.4. There is a constant $A_{1} \geq 0$ such that for any $n$, any regular sequence $\left\{\alpha_{0}, \ldots, \alpha_{n-1}\right\}$ of length $n$ in $E_{v}$, and any $z \in E_{v}$, there is an index $J$ for which $\operatorname{ord}_{v}\left(z-\alpha_{J}\right) \geq \log _{v}(n)-A_{1}$.

Proof. Suppose $z \in E_{v, i}=a_{i}+b_{i} O_{v}$, and let $n_{i}$ be the number of elements of the sequence which belong to $E_{v, i}$. Let $J$ be an index for which $\operatorname{ord}_{v}\left(z-\alpha_{J}\right)$ is maximal. If $n_{i}>0$, then $\alpha_{J} \in E_{v, i}$, and by (8.5),

$$
\operatorname{ord}_{v}\left(z-\alpha_{J}\right) \geq \operatorname{ord}_{v}\left(b_{i}\right)+\left\lfloor\log _{v}\left(n_{i}\right)\right\rfloor \geq \log _{v}(n)+\operatorname{ord}_{v}\left(b_{i}\right)+\log _{v}\left(w_{i}\right)-1 \text {. }
$$

On the other hand if $n_{i}=0$ then necessarily $w_{i} n<1$ and so

$$
\operatorname{ord}_{v}\left(z-\alpha_{J}\right) \geq-\log _{v}(R)>\log _{v}(n)-\log _{v}(R)+\log _{v}\left(w_{i}\right) .
$$

Since there are only finitely many $E_{v, i}$ the result follows.

Lemma 8.5. Let $\left\{\alpha_{0}, \ldots, \alpha_{n-1}\right\}$ be a regular sequence of length $n$ in $E_{v}$. Given $z \in E_{v}$, let $0 \leq J<n$ be an index for which $\operatorname{ord}_{v}\left(z-\alpha_{J}\right)$ is maximal. Then for any $i \neq J$,

$$
\operatorname{ord}_{v}\left(z-\alpha_{i}\right) \leq \operatorname{ord}_{v}\left(\alpha_{J}-\alpha_{i}\right) .
$$

Moreover, if $n$ is large enough that each $E_{v, h}$ contains points of the regular sequence, then for any $i \neq J$,

$$
\operatorname{ord}_{v}\left(z-\alpha_{i}\right)=\operatorname{ord}_{v}\left(\alpha_{J}-\alpha_{i}\right) .
$$

Proof. To prove the inequality, first suppose $i$ is such that $\operatorname{ord}_{v}\left(z-\alpha_{i}\right)<$ $\operatorname{ord}_{v}\left(z-\alpha_{J}\right)$. Then

$$
\operatorname{ord}_{v}\left(\alpha_{J}-\alpha_{i}\right)=\min \left(\operatorname{ord}_{v}\left(z-\alpha_{J}\right), \operatorname{ord}_{v}\left(z-\alpha_{i}\right)\right)=\operatorname{ord}_{v}\left(z-\alpha_{i}\right) .
$$

Next suppose that $i$ is an index for which $\operatorname{ord}_{v}\left(z-\alpha_{i}\right)=\operatorname{ord}_{v}\left(z-\alpha_{J}\right)$. If $\operatorname{ord}_{v}\left(z-\alpha_{i}\right)>\operatorname{ord}_{v}\left(\alpha_{J}-\alpha_{i}\right)$, then

$\operatorname{ord}_{v}\left(z-\alpha_{J}\right)=\min \left(\operatorname{ord}_{v}\left(z-\alpha_{i}\right), \operatorname{ord}_{v}\left(\alpha_{J}-\alpha_{i}\right)\right)=\operatorname{ord}_{v}\left(\alpha_{J}-\alpha_{i}\right)<\operatorname{ord}_{v}\left(z-\alpha_{i}\right)$, a contradiction. Hence $\operatorname{ord}_{v}\left(z-\alpha_{i}\right) \leq \operatorname{ord}_{v}\left(\alpha_{J}-\alpha_{i}\right)$. 
If $n$ is large enough that each $E_{v, h}$ contains points of the regular sequence, then necessarily $\alpha_{J}$ belongs to the same coset $E_{v, h}=a_{h}+b_{h} O_{v}$ as $z$. If $\alpha_{i}$ belongs to another coset $E_{v, l}=a_{l}+b_{l} O_{v}$, then

$$
\operatorname{ord}_{v}\left(z-\alpha_{i}\right)=\operatorname{ord}_{v}\left(a_{h}-a_{l}\right)=\operatorname{ord}_{v}\left(\alpha_{J}-\alpha_{i}\right) \text {. }
$$

If $\alpha_{i}$ belongs to $E_{v, h}$, let $n_{h}$ be the number of elements of the regular sequence in $E_{v, h}$. By the construction of the basic well-distributed sequence, since $\operatorname{ord}_{v}\left(z-\alpha_{J}\right)$ is maximal, by (8.5) we must have

$$
\operatorname{ord}_{v}\left(z-\alpha_{J}\right) \geq \operatorname{ord}_{v}\left(b_{h}\right)+\left\lfloor\log _{v}\left(n_{h}\right)\right\rfloor,
$$

while by (8.4), since $\alpha_{i} \neq \alpha_{J}$,

$$
\operatorname{ord}_{v}\left(\alpha_{J}-\alpha_{i}\right) \leq \operatorname{ord}_{v}\left(b_{h}\right)+\left\lfloor\log _{v}\left(n_{h}\right)\right\rfloor .
$$

Hence $\operatorname{ord}_{v}\left(z-\alpha_{i}\right) \geq \operatorname{ord}_{v}\left(\alpha_{J}-\alpha_{i}\right)$ by the ultrametric inequality. Combined with our earlier inequality, this gives $\operatorname{ord}_{v}\left(z-\alpha_{i}\right)=\operatorname{ord}_{v}\left(\alpha_{J}-\alpha_{i}\right)$.

The following lemma shows that each regular sequence of length $n$ contains "many" disjoint regular subsequences of shorter length.

Lemma 8.6. (A) For any $C_{1}>0$, there is a $C_{2}>0$ such that for all sufficiently large $n$, any regular sequence of length $n$ in $E_{v}$ contains at least $\left\lceil C_{2} \sqrt{n}\right\rceil$ pairwise disjoint subsets, each of which is a regular sequence in $E_{v}$ of length $\left\lceil C_{1} \sqrt{n}\right\rceil$.

(B) For any $C_{3}>0$, there is a $C_{4}>0$ such that for all sufficiently large $n$, if $S_{1}, \ldots, S_{Z}$ are regular sequences in $E_{v}$, where each $S_{l}$ has length $n_{l} \leq\left\lceil C_{3} \sqrt{n}\right\rceil$ and $Z \leq\left\lceil C_{4} \sqrt{n}\right\rceil$, then any regular sequence $\left\{\alpha_{i}\right\}_{0 \leq i<n}$ in $E_{v}$ contains a regular subsequence $Q=\left\{\alpha_{i_{0}}, \ldots, \alpha_{i_{W-1}}\right\}$ of length $W=\left\lceil C_{3} \sqrt{n}\right\rceil$ such that for each $\alpha_{i_{k}} \in Q$ and each $\theta \in \bigcup_{l=1}^{Z} S_{l}$,

$$
\operatorname{ord}_{v}\left(\alpha_{i_{k}}-\theta\right)<A_{0}+\log _{v}(n) .
$$

Proof. For (A), recall that for any $n$, each $E_{v, i}=a_{i}+b_{i} O_{v}$ receives $\Phi_{i}(n) \geq\left\lfloor w_{i} n\right\rfloor$ elements from a regular sequence of length $n$. Write $B_{i}=$ $\operatorname{ord}_{v}\left(b_{i}\right)$. Then for each integer $B \geq B_{i}$, these $\Phi_{i}(n)$ elements are distributed among the cosets $d+\pi_{v}^{B} O_{v}$ in $E_{v, i}$ in such a way that each coset receives at least $\left\lfloor\left\lfloor w_{i} n\right\rfloor / q^{B-B_{i}}\right\rfloor$ elements. Now take $B=\left\lceil A_{0}+\log _{v}\left(\left\lceil C_{1} \sqrt{n}\right\rceil\right)\right\rceil$. Then each coset $d+\pi_{v}^{B} O_{v}$ contained in $E_{v, i}$ receives at least

$$
\begin{aligned}
\left\lfloor\left\lfloor w_{i} n\right\rfloor / q^{B-B_{i}}\right\rfloor & \geq w_{i} n / q^{A_{0}+\log _{v}(\sqrt{n})+\log _{v}\left(C_{1}\right)+2-B_{i}}-2 \\
& =\left(w_{i} / q^{A_{0}-B_{i}+\log _{v}\left(C_{1}\right)+2}\right) \sqrt{n}-2
\end{aligned}
$$

elements from a regular sequence with length $n$, but at most one element from a regular sequence of length $\left\lceil C_{1} \sqrt{n}\right\rceil$ (by the definition of a regular sequence).

Let $C_{2}>0$ be less than the minimum of $w_{i} / q^{A_{0}-B_{i}+\log _{v}\left(C_{1}\right)+2}$ for $i=$ $1, \ldots, M$. For sufficiently large $n$, each regular sequence of length $n$ in $E_{v}$ 
contains regular subsequences of length $\left\lceil C_{1} \sqrt{n}\right\rceil$ (the initial elements form such a subsequence). And, if $B$ is as above, then for sufficiently large $n$ each coset $d+\pi_{v}^{B} O_{v}$ in $E_{v}$ receives at least $\left\lceil C_{2} \sqrt{n}\right\rceil$ elements from the regular sequence, so there are at least that many disjoint regular subsequences of length $\left\lceil C_{1} \sqrt{n}\right\rceil$ in a regular sequence of length $n$.

The proof of (B) is similar.

Products like $\prod_{j=0}^{n-1}\left(\beta_{v}(n)-\beta_{v}(j)\right)$ are analogous to factorials: by (8.3),

We claim that

$$
\operatorname{ord}_{v}\left(\prod_{j=0}^{n-1}\left(\beta_{v}(n)-\beta_{v}(j)\right)\right)=\sum_{j=1}^{n} \operatorname{val}_{q}(j) \text {. }
$$

$$
\sum_{j=1}^{n} \operatorname{val}_{q}(j)=\frac{n}{q-1}-\frac{1}{q-1} \sum_{i \geq 0} d_{i}(n) .
$$

This generalizes the familiar formula for $\operatorname{ord}_{p}(n !)$. For each $k \geq 1$, there are exactly $\left\lfloor n / q^{k}\right\rfloor$ numbers $j$ in the range $1 \leq j \leq n$ for $\operatorname{which}_{\operatorname{val}_{q}}(j) \geq k$. Hence, writing $d_{i}=d_{i}(n)$, we have

$$
\begin{aligned}
\sum_{j=1}^{n} \operatorname{val}_{q}(j) & =\sum_{k \geq 1}\left\lfloor\frac{n}{q^{k}}\right\rfloor \\
& =\left(d_{1}+d_{2} q+d_{3} q^{2}+\ldots\right)+\left(d_{2}+d_{3} q+\ldots\right)+\ldots \\
& =d_{1} \cdot \frac{q-1}{q-1}+d_{2} \cdot \frac{q^{2}-1}{q-1}+d_{3} \cdot \frac{q^{3}-1}{q-1}+\ldots \\
& =\frac{d_{0}+d_{1} q+d_{2} q^{2}+\ldots}{q-1}-\frac{d_{0}+d_{1}+d_{2}+\ldots}{q-1} \\
& =\frac{n}{q-1}-\frac{1}{q-1} \sum_{i \geq 0} d_{i}(n) .
\end{aligned}
$$

For the next lemma, it is crucial that the weights $w_{i}$ defining a regular sequence arise from the equilibrium distribution of $E_{v}$.

Lemma 8.7. There is a constant $A_{2} \geq 0$ such that for all $n$, if $\left\{\alpha_{i}\right\}_{0 \leq i<n}$ is a regular sequence of length $n$ in $E_{v}$ and $f(z)=\prod_{i=0}^{n-1}\left(z-\alpha_{i}\right)$, then:

(A) For each $J$ in the range $0 \leq J<n$, we have

$$
n V\left(E_{v}\right)-2 \log _{v}(n)-A_{2} \leq \operatorname{ord}_{v}\left(\prod_{\substack{i=0 \\ i \neq J}}^{n-1}\left(\alpha_{J}-\alpha_{i}\right)\right) \leq n V\left(E_{v}\right)+A_{2} .
$$

(B) For each $z \in E_{v}$, if $0 \leq J<n$ is such that $\operatorname{ord}_{v}\left(z-\alpha_{J}\right)$ is maximal, then

$$
n V\left(E_{v}\right)-\log _{v}(n)-A_{2} \leq \operatorname{ord}_{v}(f(z)) \leq n V\left(E_{v}\right)+A_{2}+\operatorname{ord}_{v}\left(z-\alpha_{J}\right) .
$$

In particular, $\|f\|_{E_{v}} \leq q^{-n V\left(E_{v}\right)+\log _{v}(n)+A_{2}}$. 
Proof. First consider the special case $E_{v}=O_{v}$ with $\left\{\lambda_{v}(k)\right\}=\left\{\beta_{v}(k)\right\}$. Then $V\left(O_{v}\right)=1 /(q-1)$ and $A_{0}=0$.

To prove (A), fix $J$ and note that if $i \neq J$, then $\operatorname{ord}_{v}\left(\alpha_{J}-\alpha_{i}\right)=$ $\operatorname{ord}_{v}\left(\beta_{v}(J)-\beta_{v}(i)\right)=\operatorname{val}_{q}(|J-i|)$. Hence

$$
\begin{aligned}
\operatorname{ord}_{v}\left(\prod_{\substack{i=0 \\
i \neq J}}^{n-1}\left(\alpha_{J}-\alpha_{i}\right)\right) & =\sum_{l=1}^{J} \operatorname{val}_{q}(l)+\sum_{l=1}^{n-J-1} \operatorname{val}_{q}(l) \\
& =\frac{n}{q-1}-\frac{\sum d_{i}(J)+\sum d_{i}(n-J-1)+1}{q-1} .
\end{aligned}
$$

It is easy to see that

and hence that

$$
0 \leq \sum d_{i}(J)+\sum d_{i}(n-J-1)+1 \leq 2(q-1)\left\lceil\log _{v}(n)\right\rceil
$$

$$
\frac{n}{q-1}-2 \log _{v}(n)-2 \leq \operatorname{ord}_{v}\left(\prod_{\substack{i=0 \\ i \neq J}}^{n-1}\left(\alpha_{J}-\alpha_{i}\right)\right) \leq \frac{n}{q-1} .
$$

This yields assertion (A).

To obtain (B), let $J$ be an index for which $\operatorname{ord}_{v}\left(z-\alpha_{J}\right)$ is maximal. Then

$$
\operatorname{ord}_{v}\left(z-\alpha_{J}\right) \geq\left\lfloor\log _{v}(n)\right\rfloor \geq \log _{v}(n)-1
$$

and by Lemma 8.5, using the fact that $E_{v}=O_{v}$ consists of only one coset, for each $i \neq J$,

$$
\operatorname{ord}_{v}\left(z-\alpha_{i}\right)=\operatorname{ord}_{v}\left(\alpha_{J}-\alpha_{i}\right) .
$$

Hence, adding the term $\operatorname{ord}_{v}\left(z-\alpha_{J}\right)$ to $(8.16)$, we find that

$$
\frac{n}{q-1}-\log _{v}(n)-3 \leq \operatorname{ord}_{v}(f(z)) \leq \frac{n}{q-1}+\operatorname{ord}_{v}\left(z-\alpha_{J}\right) .
$$

Now let $E_{v}$ be arbitrary: $E_{v}=\bigcup_{l=1}^{M} E_{v, l}$, where $E_{v, l}=a_{l}+b_{l} O_{v}$. We can assume $n$ is large enough that any regular sequence of length $n$ in $E_{v}$ contains elements from each $E_{v, l}$.

To prove (A), fix $J$, and let $h$ be the index for which $\alpha_{J} \in E_{v, h}$. If $l \neq h$ and $\alpha_{i} \in E_{v, l}$, then $\left|\alpha_{J}-\alpha_{i}\right|_{v}=\left|a_{l}-a_{h}\right|_{v}$, so

$$
\operatorname{ord}_{v}\left(\prod_{\alpha_{i} \in E_{v, l}}\left(\alpha_{J}-\alpha_{i}\right)\right)=\Phi_{l}(n) \operatorname{ord}_{v}\left(a_{l}-a_{h}\right) .
$$

On the other hand, the $\alpha_{i} \in E_{v, h}$ form a regular sequence of length $\Phi_{h}(n)$ in $E_{v, h}$, and are an affine transformation of a regular sequence in $O_{v}$. By (8.16),

$$
\begin{aligned}
& \Phi_{h}(n)\left(\frac{1}{q-1}+\operatorname{ord}_{v}\left(b_{h}\right)\right)-2 \log _{v}\left(\Phi_{h}(n)\right)-2-\operatorname{ord}_{v}\left(b_{h}\right) \\
\leq & \operatorname{ord}_{v}\left(\prod_{\substack{\alpha_{i} \in E_{v, h} \\
i \neq J}}\left(\alpha_{J}-\alpha_{i}\right)\right) \leq \Phi_{h}(n)\left(\frac{1}{q-1}+\operatorname{ord}_{v}\left(b_{h}\right)\right)-\operatorname{ord}_{v}\left(b_{h}\right) .
\end{aligned}
$$


For each $l$, we have $\left\lfloor w_{l} n\right\rfloor \leq \Phi_{l}(n) \leq\left\lceil w_{l} n\right\rceil$, while by (3.10),

$$
\sum_{l \neq h} w_{l} \operatorname{ord}_{v}\left(a_{l}-a_{h}\right)+w_{h}\left(\frac{1}{q-1}+\operatorname{ord}_{v}\left(b_{h}\right)\right)=V\left(E_{v}\right) .
$$

Summing the inequalities (8.18) and (8.19) and using (8.20), we find that there is a constant $A_{2}$ (which can be taken independent of $h$ ) such that

$$
n V\left(E_{v}\right)-2 \log _{v}(n)-A_{2} \leq \operatorname{ord}_{v}\left(\prod_{\substack{i=0 \\ i \neq J}}^{n-1}\left(\alpha_{J}-\alpha_{i}\right)\right) \leq n V\left(E_{v}\right)+A_{2} .
$$

This yields (A). We obtain (B) as before, possibly after increasing $A_{2}$.

We can now state the basic lemma governing the patching process.

LEMMA 8.8. Let $\left\{\alpha_{i}\right\}_{0 \leq i<m}$ be a regular sequence of length $m$ in $E_{v}$, and put $f(z)=\prod_{i=0}^{m-1}\left(z-\alpha_{i}\right)$. Then for any $M \geq A_{0}+\log _{v}(m)$, and any $\Delta \in K_{v}$ satisfying

$$
\operatorname{ord}_{v}(\Delta) \geq m V\left(E_{v}\right)+A_{2}+M,
$$

the roots of $f^{*}(z):=f(z)+\Delta$ again form a regular sequence of length $m$ in $E_{v}$, and if we write $f^{*}(z)=\prod_{i=0}^{m-1}\left(z-\alpha_{i}^{*}\right)$, the roots $\alpha_{i}^{*}$ can be uniquely labeled in such a way that

$$
\operatorname{ord}_{v}\left(\alpha_{i}^{*}-\alpha_{i}\right) \geq M
$$

for each $i$. More generally, if $E_{v} \subset B(0, R)$, let $\Delta(z) \in K_{v}[z]$ be any polynomial of degree $<m$ such that

$$
\operatorname{ord}_{v}(\Delta(z)) \geq m V\left(E_{v}\right)+A_{2}+M
$$

for all $z \in B(0, R)$. Then the same assertions hold for $f^{*}(z):=f(z)+\Delta(z)$.

Proof. Fix a root $\alpha_{J}$ of $f(z)$, and expand $f(z), f^{*}(z)$ and $\Delta(z)$ about $\alpha_{J}$, writing

$$
\begin{aligned}
f(z) & =b_{1}\left(z-\alpha_{J}\right)+b_{2}\left(z-\alpha_{J}\right)^{2}+\ldots+b_{m-1}\left(z-\alpha_{J}\right)^{m-1}+\left(z-\alpha_{J}\right)^{m}, \\
f^{*}(z) & =c_{0}+c_{1}\left(z-\alpha_{J}\right)+c_{2}\left(z-\alpha_{J}\right)^{2}+\ldots+\left(z-\alpha_{J}\right)^{m}, \\
\Delta(z) & =\Delta_{0}+\Delta_{1}\left(z-\alpha_{J}\right)+\ldots+\Delta_{m-1}\left(z-\alpha_{J}\right)^{m-1} .
\end{aligned}
$$

Here $b_{1}= \pm \prod_{i \neq J}\left(\alpha_{J}-\alpha_{i}\right)$, so by Lemma 8.7(A), $\operatorname{ord}_{v}\left(b_{1}\right) \leq m V\left(E_{v}\right)+A_{2}$. For each $k \geq 2$,

$$
b_{k}= \pm b_{1} \sum_{\substack{i_{1}<\ldots<i_{k-1} \\ \text { each } i_{j} \neq J}} \frac{1}{\left(\alpha_{J}-\alpha_{i_{1}}\right) \ldots\left(\alpha_{J}-\alpha_{i_{k-1}}\right)}
$$

and so by (8.9),

$$
\operatorname{ord}_{v}\left(b_{k}\right)>\operatorname{ord}_{v}\left(b_{1}\right)-(k-1)\left(A_{0}+\log _{v}(m)\right) .
$$


On the other hand, by the hypothesis on $\Delta(z)$ and an easy estimate using the maximum principle, for each $k \geq 0$,

$$
\operatorname{ord}_{v}\left(\Delta_{k}\right) \geq\left(m V\left(E_{v}\right)+A_{2}+M\right)+k \log _{v}(R) .
$$

Here $A_{0} \geq-\log _{v}(R)$ by Remark 8.1, and $M \geq A_{0}+\log _{v}(m)>A_{0}$ (assuming $m \geq 2$, which is permissible since the lemma is trivial when $m=1$ ), so for each $k \geq 1$,

$$
\operatorname{ord}_{v}\left(\Delta_{k}\right)>\operatorname{ord}_{v}\left(b_{1}\right)-(k-1)\left(A_{0}+\log _{v}(m)\right) .
$$

Now consider the Newton polygon of $f^{*}(z)$, expanded about $\alpha_{J}$. By the estimates above, we have

$$
\begin{aligned}
& \operatorname{ord}_{v}\left(c_{0}\right)=\operatorname{ord}_{v}\left(\Delta_{0}\right) \geq \operatorname{ord}_{v}\left(b_{1}\right)+M, \\
& \operatorname{ord}_{v}\left(c_{1}\right)=\operatorname{ord}_{v}\left(b_{1}\right), \\
& \operatorname{ord}_{v}\left(c_{k}\right)>\operatorname{ord}_{v}\left(b_{1}\right)-(k-1)\left(A_{0}+\log _{v}(m)\right) \quad \text { for all } k \geq 2 .
\end{aligned}
$$

Thus the Newton polygon has a break at the point $\left(1, \operatorname{ord}_{v}\left(c_{1}\right)\right)$, and $f^{*}(z)$ has a unique root $\alpha_{J}^{*}$ for which

$$
\operatorname{ord}_{v}\left(\alpha_{J}^{*}-\alpha_{J}\right) \geq M \text {. }
$$

By its uniqueness, $\alpha_{J}^{*}$ belongs to $K_{v}$, and by Remark 8.1 it belongs to $E_{v}$, since $M \geq A_{0}$.

Since this is true for each $J$, it follows that $\left\{\alpha_{J}^{*}\right\}_{0 \leq J<m}$ is a regular sequence of length $m$ in $E_{v}$.

Recall that numbers $h_{v}$ and $r_{v}$ were fixed at the beginning of the proof, satisfying

$$
0<h_{v}<r_{v}<\gamma\left(E_{v}\right)=q^{-V\left(E_{v}\right)} .
$$

We are constructing a constant $L$ subject to various local conditions; for this place $v$ the condition will be that

$$
L \cdot\left(-\log _{v}\left(h_{v} / r_{v}\right)\right) \geq A_{2}+A_{0}+\log _{v}(L)+2 .
$$

The line on the left side of (8.21) meets the logarithmic curve on the right side at most twice. Since $\left(A_{2}+A_{0}+2\right) \log \left(q_{v}\right) \geq 2 \log (2)>1$, if there are two intersections, one occurs for $L<1$. Hence there is a unique least positive integer $L$ for which (8.21) holds; for all $l \geq L$,

$$
l \cdot\left(-\log _{v}\left(h_{v} / r_{v}\right)\right) \geq A_{2}+A_{0}+\left\lceil\log _{v}(l)\right\rceil .
$$

Taking $M_{v}=q_{v}^{A_{2}+A_{0}+2},(8.21)$ is equivalent to

$$
L\left(\frac{h_{v}}{r_{v}}\right)^{L} M_{v} \leq 1,
$$


which is the condition stated in Proposition 8.1. The constant $B_{v}$ in Proposition 8.1 will be defined by

$$
-\log _{v}\left(B_{v}\right)=\max _{1 \leq k \leq L}\left(\left\lceil k V\left(E_{v}\right)+A_{2}+A_{0}+\left\lceil\log _{v}(k)\right\rceil\right\rceil\right) .
$$

Proof of Proposition 8.1. Given $n$, choose a regular sequence $\left\{\alpha_{l}\right\}_{0 \leq l<n}$ of length $n$ in $E_{v}$, and take the initial patching polynomial to be

$$
u_{v}^{(0)}(z)=\prod_{l=0}^{n-1}\left(z-\alpha_{l}\right) .
$$

In the construction, if $n$ is sufficiently large, we will successively modify $u_{v}^{(0)}(z)$ to polynomials $u_{v}^{(1)}(z), u_{v}^{(2)}(z), \ldots, u_{v}^{(n)}(z)$, where

$$
u_{v}^{(k)}(z)=z^{n}+\sum_{i=1}^{n} c_{v, i}^{(k)} z^{n-i}=\prod_{l=0}^{n-1}\left(z-\alpha_{l}^{(k)}\right) .
$$

At each step, all the roots of $u_{v}^{(k)}(z)$ will belong to $E_{v}$. There will be a well-defined correspondence between the roots of $u_{v}^{(k-1)}(z)$ and those of $u_{v}^{(k)}(z)$, so that a given root $\alpha_{l}^{(0)}=\alpha_{l}$ of $u_{v}^{(0)}(z)$ is successively modified to $\alpha_{l}^{(1)}, \alpha_{l}^{(2)}, \ldots, \alpha_{l}^{(n)}$. Because of this, if $S$ is a subset of the original regular sequence $\left\{\alpha_{0}, \ldots, \alpha_{n-1}\right\}$, it makes sense to speak of the set

$$
\left\{\alpha_{l}^{(k)}: \alpha_{l} \in S\right\} .
$$

The $k-1$ high-order coefficients of $u_{v}^{(k)}(z)$ will be the same as those of $u_{v}^{(k-1)}(z)$, while $c_{v, k}^{(k)}=c_{v, k}^{(k-1)}+\Delta_{v}^{(k)}$. In applications, the numbers $\Delta_{v}^{(k)}$ will be chosen on the basis of global considerations.

The construction has five phases. The first two phases involve "building up strength" regarding the amount of movement which a patching correction $c_{v, k}^{(k)}=c_{v, k}^{(k-1)}+\Delta_{v}^{(k)}$ causes in the roots of $u_{v}^{(k-1)}(z)$. These initial steps will cause large movement in some of the roots, destroying the property that the roots form a regular sequence of length $n$. The last three phases compensate for this.

The basic idea in the first two phases is as follows. To pass from $u_{v}^{(k-1)}(z)$ to $u_{v}^{(k)}(z)$, we take a subset $S=\left\{\alpha_{i_{0}}^{(k-1)}, \ldots, \alpha_{i_{k-1}}^{(k-1)}\right\}$ of the roots $u_{v}^{(k-1)}(z)$ which forms a regular sequence of length $k$. Put

$$
\begin{aligned}
f_{k}(z) & =\prod_{\alpha_{l}^{(k-1)} \in S}\left(z-\alpha_{l}^{(k-1)}\right), \\
w_{v}^{(k)}(z) & =\prod_{\alpha_{l}^{(k-1)} \notin S}\left(z-\alpha_{l}^{(k-1)}\right)=u_{v}^{(k-1)}(z) / f_{k}(z),
\end{aligned}
$$


and set

$$
u_{v}^{(k)}(z)=u_{v}^{(k-1)}(z)+\Delta_{v}^{(k)} w_{v}^{(k)}(z)=\left(f_{k}(z)+\Delta_{v}^{(k)}\right) w_{v}^{(k)}(z) .
$$

We then apply Lemma 8.8 to $f_{k}(z)$ and $\Delta_{v}^{(k)}$, checking that the condition on $\Delta_{v}^{(k)}$ assures that the roots of $f_{k}^{*}(z)=f_{k}(z)+\Delta_{v}^{(k)}$ belong to $E$. Since $w_{v}^{(k)}(z)$ is monic of degree $n-k$, the step (8.26) changes $c_{v, k}^{(k-1)}$ to $c_{v, k}^{(k)}=c_{v, k}^{(k-1)}+\Delta_{v}^{(k)}$ and accomplishes the desired patching correction in $u_{v}^{(k-1)}(z)$.

Write $\Omega=\left\{\alpha_{l}\right\}_{0 \leq l<n}$ for the initial regular sequence of length $n$, and let $T$ be the least positive integer such that

$$
T \cdot\left(-\log _{v}\left(h_{v} / r_{v}\right)\right) \geq A_{2}+A_{0}+\log _{v}(n)+2 .
$$

Just as in (8.21), for all $k \geq T$ we have

$$
k \cdot\left(-\log _{v}\left(h_{v} / r_{v}\right)\right) \geq A_{2}+A_{0}+\left\lceil\log _{v}(n)\right\rceil .
$$

There is a constant $A_{3}$ such that for all sufficiently large $n$,

$$
T \leq A_{3} \log _{v}(n) .
$$

The construction will require us to choose $T$ pairwise disjoint regular subsequences from $\Omega$, one of each length $1,2, \ldots, T$. By Lemma 8.6(A) this can be done if $n$ is large enough, which we henceforth assume. Let $S_{1}, \ldots, S_{T}$ be these subsequences. Only roots $\alpha_{l} \in S_{k}$ are moved in the $k$ th step of the construction, for $k=1, \ldots, T$.

Phase 1. Patching the high-order coefficients, $k=1, \ldots, L$. Inductively suppose $u_{v}^{(k-1)}(z)$ has been determined, and that its roots, apart from the $\alpha_{l}$ in $S_{1}, \ldots, S_{k-1}$, are the same as those of $u_{v}^{(0)}(z)$. Take $S=S_{k}$, and define $f_{k}(z), w_{v}^{(k)}(z)$, and $u_{v}^{(k)}(z)$ as in (8.24)-(8.26). Since $\left|\Delta_{v}^{(k)}\right|_{v} \leq B_{v}$ it follows from (8.23) that

$$
\operatorname{ord}_{v}\left(\Delta_{v}^{(k)}\right) \geq k V\left(E_{v}\right)+A_{2}+A_{0}+\left\lceil\log _{v}(k)\right\rceil
$$

and so by Lemma 8.8,

$$
f_{k}^{*}(z)=f_{k}(z)+\Delta_{v}^{(k)}=\prod_{\alpha_{l} \in S_{k}}\left(z-\alpha_{l}^{*}\right)
$$

is a polynomial whose roots belong to $E_{v}$; moreover Lemma 8.8 gives a unique correspondence between the roots $\alpha_{l}$ and the $\alpha_{l}^{*}$ such that

$$
\operatorname{ord}_{v}\left(\alpha_{l}^{*}-\alpha_{l}\right) \geq A_{0}+\left\lceil\log _{v}(k)\right\rceil .
$$

In passing from $u_{v}^{(k-1)}(z)$ to $u_{v}^{(k)}(z)$, the roots $\alpha_{l} \in S_{k}$ are replaced by the $\alpha_{l}^{*}$, and the remaining roots are unchanged; $S_{k}^{*}=\left\{\alpha_{l}^{*}\right\}_{\alpha_{l} \in S_{k}}$ is a regular sequence of length $k$ in $E_{v}$.

Phase 2. Patching the coefficients for $k=L+1, \ldots, T$. In this phase, the construction is exactly the same as in Phase 1 , except that now $\left|\Delta_{v}^{(k)}\right|_{v}$ 
$\leq h_{v}^{k}$. By $(8.22)$,

$$
\operatorname{ord}_{v}\left(\Delta_{v}^{(k)}\right) \geq k V\left(E_{v}\right)+A_{0}+A_{2}+\left\lceil\log _{v}(k)\right\rceil
$$

and so Lemma 8.8 applies as before. In passing from $u_{v}^{(k-1)}(z)$ to $u_{v}^{(k)}(z)$, the roots in $S_{k}$ are replaced by the roots in $S_{k}^{*}=\left\{\alpha_{l}^{*}\right\}_{\alpha_{l} \in S_{k}}$ forming a new regular sequence of length $k$, and the remaining roots are unchanged.

Phase 3. Moving the roots apart. Put $\Omega_{0}=\Omega \backslash\left(S_{1} \cup \ldots \cup S_{T}\right)$. At this point, we have a polynomial $u_{v}^{(T)}(z)$ whose roots compose the set $\Omega_{0} \cup S_{1}^{*} \cup$ $\ldots \cup S_{T}^{*}$. Although the roots within $\Omega_{0}$ and within each $S_{i}^{*}$ are well-separated from each other in the sense that each pair $\alpha_{k}^{(T)} \neq \alpha_{l}^{(T)}$ of such roots satisfies $\operatorname{ord}_{v}\left(\alpha_{k}^{(T)}-\alpha_{l}^{(T)}\right)<A_{0}+\log _{v}(n)$, some roots in $S_{i}^{*}$ may be very near to roots in $\Omega_{0}$ or roots in other $S_{j}^{*}$. Therefore, we pause to move them apart. Put

$$
\begin{aligned}
B= & \left\lceil(T-1)\left(V\left(E_{v}\right)+\log _{v}(R)\right)\right\rceil \\
& +\left\lceil\max \left(0, V\left(E_{v}\right)\right)+A_{2}+A_{0}+\left\lceil\log _{v}(n)\right\rceil\right\rceil .
\end{aligned}
$$

Since $B \geq A_{0}+\left\lceil\log _{v}(n)\right\rceil$, each coset of the form $d+\pi_{v}^{B} O_{v}$ in $E_{v}$ contains at most $T+1$ elements from $\Omega_{0} \cup S_{1}^{*} \cup \ldots \cup S_{T}^{*}$. Let $D \geq 0$ be the smallest integer for which

$$
q^{D} \geq T+1 .
$$

Then it is possible to move the roots $\alpha_{l}^{*}$ in $S_{1}^{*} \cup \ldots \cup S_{T}^{*}$ to numbers $\alpha_{l}^{\#}=$ $\alpha_{l}^{*}+\varepsilon_{l}$, by adding on quantities $\varepsilon_{l} \in K_{v}$ with $\operatorname{ord}_{v}\left(\varepsilon_{l}\right) \geq B$, in such a way that each $\alpha_{l}^{\#}$ satisfies

$$
\begin{cases}\operatorname{ord}_{v}\left(\alpha_{l}^{\#}-\alpha_{j}^{\#}\right) \leq B+D & \text { for all } \alpha_{j}^{\#} \neq \alpha_{l}^{\#}, \\ \operatorname{ord}_{v}\left(\alpha_{l}^{\#}-\alpha_{j}\right) \leq B+D & \text { for all } \alpha_{j} \in \Omega_{0} .\end{cases}
$$

Let $S_{k}^{\#}$ be the set of roots $\alpha_{l}^{\#}$ obtained from $S_{k}^{*}$; each $S_{k}^{\#}$ is a regular sequence of length $k$ in $E_{v}$. Since $T \leq A_{3} \log _{v}(n)$, there is a constant $A_{4}$ such that for all sufficiently large $n$,

$$
B+D<A_{4} \log _{v}(n) .
$$

Put

$$
\widetilde{u}_{v}^{(T)}(z)=\prod_{k=1}^{T}\left(\prod_{\alpha_{l}^{\#} \in S_{k}^{\#}}\left(z-\alpha_{l}^{\#}\right)\right) \cdot \prod_{\alpha_{l} \in \Omega_{0}}\left(z-\alpha_{l}\right) .
$$

The roots of $\tilde{u}_{v}^{(T)}(z)$ are "moderately well-separated" in the sense that (for sufficiently large $n$ ) any two of its roots $\widetilde{\alpha}_{l} \neq \widetilde{\alpha}_{j}$ satisfy $\operatorname{ord}_{v}\left(\widetilde{\alpha}_{l}-\widetilde{\alpha}_{j}\right)$ $\leq A_{4} \log _{v}(n)$. However, in general it will not have the same $T$ high-order 
coefficients as $u_{v}^{(T)}(z)$. Write

$$
\widetilde{u}_{v}^{(T)}(z)=z^{n}+\sum_{l=1}^{n} \widetilde{c}_{v, l}^{(T)} z^{n-l}
$$

and for each $l \leq T$, put

$$
\delta_{l}=c_{v, l}^{(T)}-\widetilde{c}_{v, l}^{(T)} .
$$

Considering the expansions of the $c_{v, l}^{(T)}$ and $\widetilde{c}_{v, l}^{(T)}$ as symmetric functions in the roots of $u_{v}^{(T)}(z)$ and $\widetilde{u}_{v}^{(T)}(z)$, we see that

$$
\operatorname{ord}_{v}\left(\delta_{l}\right) \geq B-(l-1) \log _{v}(R) .
$$

To change the $T$ high-order coefficients of $\widetilde{u}_{v}^{(T)}(z)$ back to those of $u_{v}^{(T)}(z)$, we move a second set of roots so as to compensate for the first.

For this purpose, choose a regular subsequence $Q_{T}$ of length $T$ from $\Omega_{0}$, which has the property that for each $\alpha_{i} \in Q_{T}$, and each $\alpha_{j}^{\#} \in S_{1}^{\#} \cup \ldots \cup S_{T}^{\#}$,

$$
\operatorname{ord}_{v}\left(\alpha_{i}-\alpha_{j}^{\#}\right)<A_{0}+\log _{v}(n) .
$$

That is, $Q_{T}$ is a regular sequence in $E_{v}$ of length $T$, contained in the original regular sequence $\Omega$ of length $n$, whose elements are "well-separated" from elements in $S_{1}, \ldots, S_{T}$ and $S_{1}^{\#}, \ldots, S_{T}^{\#}$. If $n$ is sufficiently large, the existence of such a subsequence follows from Lemma 8.6(B).

We now need a lemma.

Lemma 8.9. Let $h(z) \in K_{v}[z]$ be a monic polynomial of degree $N$ whose roots belong to $B(0, R)$. Then for any $m \geq 0$, there is a monic polynomial $g_{m}(z) \in K_{v}[z]$ of degree $m$, with $\left\|g_{m}(z)\right\|_{B(0, R)} \leq R^{m}$, such that $g_{m}(z) h(z)$ has the form

$$
g_{m}(z) h(z)=z^{N+m}+\sum_{k=1}^{N} d_{k} z^{N-k} .
$$

Proof. Write $h(z)=z^{N}+\sum_{i=1}^{N} h_{i} z^{N-i}$; by the hypothesis on the roots of $h(z)$, we have $\left|h_{i}\right|_{v} \leq R^{i}$ for all $i$. Similarly, expand $g_{m}(z)=z^{m}+$ $\sum_{j=1}^{m} \gamma_{j} z^{N-j}$ with undetermined coefficients. For $g_{m}(z) h(z)$ to have the desired form, we must have

$$
\left\{\begin{array}{l}
\gamma_{1}=-h_{1}, \\
\gamma_{2}=-h_{1} \gamma_{1}-h_{2}, \\
\vdots \\
\gamma_{m}=-h_{1} \gamma_{m-1}-h_{2} \gamma_{m-2}-\ldots-h_{m},
\end{array}\right.
$$

where we take $h_{k}=0$ if $k>N$. These equations recursively determine $\gamma_{1}, \ldots, \gamma_{m}$; moreover one sees inductively that $\left|\gamma_{j}\right|_{v} \leq R^{j}$ for each $j$. This in turn yields $\left\|g_{m}(z)\right\|_{B(0, R)} \leq R^{m}$. 
To apply this, take $N=n-T$ and put

$$
h(z)=\widetilde{u}_{v}^{(T)}(z) / \prod_{\alpha_{l} \in Q_{T}}\left(z-\alpha_{l}\right), \quad \widetilde{f}_{T}(z)=\prod_{\alpha_{l} \in Q_{T}}\left(z-\alpha_{l}\right) .
$$

With the $g_{j}(z)$ from the lemma, put

$$
\Delta(z)=\sum_{l=1}^{T} \delta_{l} g_{T-l}(z)
$$

By (8.29), (8.30) and the bounds $\left\|g_{T-l}(z)\right\|_{B(0, R)} \leq R^{T-l}$,

$$
\begin{aligned}
\operatorname{ord}_{v}(\Delta(z)) & \geq B-(T-1) \log _{v}(R) \\
& \geq T V\left(E_{v}\right)+A_{2}+A_{0}+\left\lceil\log _{v}(n)\right\rceil
\end{aligned}
$$

for all $z \in B(0, R)$.

Now define

$$
\bar{u}_{v}^{(T)}(z)=\widetilde{u}_{v}^{(T)}(z)+\Delta(z) h(z)=\left(\widetilde{f}_{T}(z)+\Delta(z)\right) h(z) .
$$

By Lemma 8.9 the high-order coefficients of $\bar{u}_{v}^{(T)}(z)$ are $c_{v, l}^{(T)}$ for $l=1, \ldots, T$. On the other hand, $\widetilde{f}_{T}(z)$ and $\Delta(z)$ satisfy the conditions of Lemma 8.8 , so by $(8.32)$,

$$
\widetilde{f}_{T}^{*}(z)=\widetilde{f}_{T}(z)+\Delta(z)=\prod_{\alpha_{l} \in Q_{T}}\left(z-\alpha_{l}^{*}\right)
$$

is a polynomial whose roots belong to $E_{v}$ and can uniquely be put in correspondence with the $\alpha_{l} \in Q_{T}$ in such a way that

$$
\operatorname{ord}_{v}\left(\alpha_{l}^{*}-\alpha_{l}\right) \geq A_{0}+\left\lceil\log _{v}(n)\right\rceil
$$

for all $i$. By (8.31) these $\alpha_{l}^{*}$ have not moved nearer to any of the $\alpha_{j}^{\#}$. Put

$$
S_{0}=\left(\Omega_{0} \backslash Q_{T}\right) \cup\left\{\alpha_{l}^{*}: \alpha_{l} \in Q_{T}\right\} .
$$

Then $S_{0}$ is a subset of a regular sequence of length $n$ in $E_{v}$ (indeed, $S_{0} \cup$ $S_{1} \cup \ldots \cup S_{T}$ is such a sequence), and the roots of $\bar{u}_{v}^{(T)}(z)$ form the set $S_{0} \cup S_{1}^{\#} \cup \ldots \cup S_{T}^{\#}$, where each $S_{k}^{\#}$ is a regular sequence of length $k$ in $E_{v}$. Furthermore each pair of distinct roots $\bar{\alpha}_{i}, \bar{\alpha}_{j}$ of $\bar{u}_{v}^{(T)}(z)$ satisfies $\operatorname{ord}_{v}\left(\bar{\alpha}_{i}-\bar{\alpha}_{j}\right)$ $<A_{4} \log _{v}(n)$.

Replace $u_{v}^{(T)}(z)$ by $\bar{u}_{v}^{(T)}(z)$.

Phase 4. Carry on. By (8.28), at the end of Phase 2 the construction reached a point that further patching moves roots only by quantities $\varepsilon$ with $\operatorname{ord}_{v}(\varepsilon) \geq A_{0}+\left\lceil\log _{v}(n)\right\rceil$, preserving their position in a regular sequence of length $n$. In Phase 3, we arranged that $\operatorname{ord}_{v}\left(\alpha_{i}^{(T)}-\alpha_{j}^{(T)}\right)<A_{4} \log _{v}(n)$ for all $i \neq j$. The purpose of Phase 4 is to carry on the construction, using the basic 
patching lemma, until further patching moves roots by quantities $\varepsilon$ with $\operatorname{ord}_{v}(\varepsilon) \geq\left\lceil A_{4} \log _{v}(n)\right\rceil$, meaning that the "delicate" roots in $S_{1}^{\#} \cup \ldots \cup S_{T}^{\#}$ can again be included in the patching process.

To motivate the duration of Phase 4, we need the following generalization of the basic patching lemma, which will be used in Phase 5 .

Lemma 8.10. Let $\Theta=\left\{\theta_{i}\right\}_{0<i<n} \subset E_{v}$ be a sequence obtained by perturbing a regular sequence $\Omega=\left\{\alpha_{i}\right\}_{0 \leq i<n}$ of length $n$; assume $\Theta$ can be partitioned into disjoint subsets $\Theta_{0} \cup \Theta_{1} \cup \ldots \cup \Theta_{Z}$ such that for each $l=1, \ldots, Z$, $\Theta_{l}$ is a regular sequence of length $n_{l}$ in $E_{v}$. Suppose $n$ is large enough that

$$
\log _{v}(n) \geq A_{0}+A_{1}+\max _{1 \leq l \leq Z}\left(\log _{v}\left(n_{l}\right)\right) .
$$

Furthermore, suppose that

$$
\operatorname{ord}_{v}\left(\theta_{i}-\alpha_{i}\right) \geq \begin{cases}A_{0}+\log _{v}(n) & \text { for each } \theta_{i} \in \Theta_{0}, \\ A_{0}+\log _{v}\left(n_{l}\right) & \text { for each } \theta_{i} \in \Theta_{l} \text { with } l \geq 1 .\end{cases}
$$

Finally, suppose that for some $M \geq A_{0}+\log _{v}(n)$, all $\theta_{i} \neq \theta_{j}$ in $\Theta$ satisfy $\operatorname{ord}_{v}\left(\theta_{i}-\theta_{j}\right)<M$. Given $k \leq n$, put $f_{k}(z)=\prod_{i=0}^{k-1}\left(z-\theta_{i}\right)$. Then for any $\Delta \in K_{v}$ with

$$
\operatorname{ord}_{v}(\Delta) \geq k V\left(E_{v}\right)+A_{2}+Z \cdot\left(M+\log _{v}(R)\right)+M,
$$

the roots of

$$
f_{k}^{*}(z):=f_{k}(z)+\Delta=\prod_{i=0}^{k-1}\left(z-\theta_{i}^{*}\right)
$$

belong to $E_{v}$ and can be uniquely labeled in such a way that

$$
\operatorname{ord}_{v}\left(\theta_{i}^{*}-\theta_{i}\right) \geq M
$$

for each $i=0, \ldots, m-1$.

Proof. Fix a root $\theta_{J}$ of $f_{k}(z)$, and expand

$$
f_{k}(z)=b_{1}\left(z-\theta_{J}\right)+b_{2}\left(z-\theta_{J}\right)^{2}+\ldots+b_{k-1}\left(z-\theta_{J}\right)^{k-1}+\left(z-\theta_{J}\right)^{k} .
$$

Here $b_{1}= \pm \prod_{i \neq J}\left(\theta_{J}-\theta_{i}\right)$, and for each $j \geq 2$,

$$
b_{j}= \pm b_{1} \sum_{\substack{i_{1}<\ldots<i_{j-1}<m \\ \text { each } i_{h} \neq J}} \frac{1}{\left(\theta_{J}-\theta_{i_{1}}\right) \ldots\left(\theta_{J}-\theta_{i_{j-1}}\right)} .
$$

By our hypothesis, for each $j \geq 2$,

$$
\operatorname{ord}_{v}\left(b_{j}\right)>\operatorname{ord}_{v}\left(b_{1}\right)-(j-1) M .
$$

We now seek an upper bound for $\operatorname{ord}_{v}\left(b_{1}\right)$. Let $I$ be an index, $0 \leq I<n$, for which $\operatorname{ord}_{v}\left(\theta_{J}-\alpha_{I}\right)$ is maximal. By Lemma 8.5 and the definition of a 
regular sequence, for each $i \neq I$,

$$
\operatorname{ord}_{v}\left(\theta_{J}-\alpha_{i}\right) \leq \operatorname{ord}_{v}\left(\alpha_{I}-\alpha_{i}\right)<A_{0}+\log _{v}(n) .
$$

Hence by (8.33), for each $\theta_{i} \in \Theta_{0}$, with $i \neq I, J$,

$$
\operatorname{ord}_{v}\left(\theta_{J}-\theta_{i}\right) \leq \operatorname{ord}_{v}\left(\alpha_{I}-\alpha_{i}\right) .
$$

Fix $l \geq 1$, and let $I_{l}$ be an index such that $\operatorname{ord}_{v}\left(\theta_{J}-\theta_{I_{l}}\right)$ is maximal among the $\operatorname{ord}_{v}\left(\theta_{J}-\theta_{i}\right)$ with $\theta_{i} \in \Theta_{l}$. Since $\Theta_{l}$ is a regular sequence of length $n_{l}$, for each $\theta_{i} \in \Theta_{l}, i \neq I_{l}$,

$$
\operatorname{ord}_{v}\left(\theta_{I_{l}}-\theta_{i}\right)<A_{0}+\log _{v}\left(n_{l}\right) .
$$

Furthermore Lemma 8.5 gives

$$
\operatorname{ord}_{v}\left(\theta_{J}-\theta_{i}\right) \leq \operatorname{ord}_{v}\left(\theta_{I_{l}}-\theta_{i}\right) .
$$

By Lemma 8.4 and our hypothesis on $n$,

$$
\operatorname{ord}_{v}\left(\theta_{J}-\alpha_{I}\right) \geq \log _{v}(n)-A_{1} \geq A_{0}+\log _{v}\left(n_{l}\right)
$$

so using (8.33) we find that for each $\theta_{i} \in \Theta_{l}$ with $i \neq I_{l}$,

$$
\operatorname{ord}_{v}\left(\theta_{J}-\theta_{i}\right)=\operatorname{ord}_{v}\left(\alpha_{I}-\alpha_{i}\right) .
$$

To estimate $\operatorname{ord}_{v}\left(b_{1}\right)$, we now consider two possibilities. If $\theta_{J} \in \Theta_{0}$, then $I=J$, and we have

$$
\begin{aligned}
\operatorname{ord}_{v}\left(b_{1}\right)= & \sum_{\substack{i=0 \\
i \neq J}}^{k-1} \operatorname{ord}_{v}\left(\theta_{J}-\theta_{i}\right) \\
= & \sum_{\substack{i=0 \\
i \neq J, I_{1}, \ldots, I_{Z}}}^{k-1} \operatorname{ord}_{v}\left(\theta_{J}-\theta_{i}\right)+\sum_{i \in\left\{I_{1}, \ldots, I_{Z}\right\}} \operatorname{ord}_{v}\left(\theta_{J}-\theta_{i}\right) \\
= & \sum_{\substack{i=0 \\
i \neq I}}^{k-1} \operatorname{ord}_{v}\left(\alpha_{I}-\alpha_{i}\right)-\sum_{i \in\left\{I_{1}, \ldots, I_{Z}\right\}} \operatorname{ord}_{v}\left(\alpha_{I}-\alpha_{i}\right) \\
& +\sum_{i \in\left\{I_{1}, \ldots, I_{Z}\right\}} \operatorname{ord}_{v}\left(\theta_{J}-\theta_{i}\right) \\
\leq & k V\left(E_{v}\right)+A_{2}+Z \cdot\left(M+\log _{v}(R)\right) .
\end{aligned}
$$

On the other hand, if $\theta_{J} \in \Theta_{l}$ for some $l \geq 1$, then $I_{l}=J$; I may or may not equal one of the $I_{l}$. In any case the sets $\left\{I, I_{1}, \ldots, I_{Z}\right\} \backslash\{I\}$ and $\left\{I, I_{1}, \ldots, I_{Z}\right\} \backslash\{J\}$ have the same number of elements, either $Z-1$ or $Z$ according as $I=I_{l}$ for some $l \geq 1$, or not. We have 


$$
\begin{aligned}
\operatorname{ord}_{v}\left(b_{1}\right)= & \sum_{\substack{i=0 \\
i \neq J}}^{k-1} \operatorname{ord}_{v}\left(\theta_{J}-\theta_{i}\right) \\
= & \sum_{\substack{i=0 \\
i \neq I, I_{1}, \ldots, I_{Z}}}^{k-1} \operatorname{ord}_{v}\left(\theta_{J}-\theta_{i}\right)+\sum_{\substack{i \in\left\{I, I_{1}, \ldots, I_{Z}\right\} \\
i \neq J}} \operatorname{ord}_{v}\left(\theta_{J}-\theta_{i}\right) \\
= & \sum_{i=0}^{k-1} \operatorname{ord}_{v}\left(\alpha_{I}-\alpha_{i}\right)-\sum_{i \in\left\{I_{i \neq I}, \ldots, I_{Z}\right\}} \operatorname{ord}_{v}\left(\alpha_{I}-\alpha_{i}\right) \\
& +\sum_{i \in\left\{I, I_{1}, \ldots, I_{Z}\right\}} \operatorname{ord}_{v}\left(\theta_{J}-\theta_{i}\right) \\
\leq & k V\left(E_{v}\right)+A_{2}+Z \cdot\left(M+\log _{v}(R)\right)
\end{aligned}
$$

once again.

Since $f_{k}^{*}(z)=f_{k}(z)+\Delta$, and $\operatorname{ord}_{v}(\Delta) \geq k V\left(E_{v}\right)+A_{2}+Z \cdot(M+$ $\left.\log _{v}(R)\right)+M$, by (8.34), (8.37) and (8.38) the Newton polygon of $f_{k}^{*}(z)$, expanded about $\theta_{J}$, has a break at the point $\left(1, \operatorname{ord}_{v}\left(b_{1}\right)\right)$ and there is a unique root $\theta_{J}^{*}$ of $f_{k}^{*}(z)$ for which

$$
\operatorname{ord}_{v}\left(\theta_{J}^{*}-\theta_{J}\right) \geq M \text {. }
$$

This holds for every $J$, so the lemma is established.

We will apply Lemma 8.10 with $Z=T<A_{3} \log _{v}(n)$, the sets $\Theta_{l}$ for $l \geq 1$ being the $S_{l}^{\#}$ with length $n_{l}=l \leq T$, and with $M=A_{4} \log _{v}(n)$. Let $W$ be the least positive integer such that

$$
W \cdot\left(-\log _{v}\left(h_{v} / r_{v}\right)\right) \geq A_{2}+T \cdot\left(M+\log _{v}(R)\right)+M+2 .
$$

As in (8.21), a similar inequality holds for each $k \geq W$. There is a constant $A_{5}$ such that $W \leq A_{5}\left(\log _{v}(n)\right)^{2}$ for all sufficiently large $n$. The purpose of Phase 4 is to carry out the patching process for $k=T+1, \ldots, W$.

For this, choose a regular subsequence $Q_{W}$ of length $W$ in $E_{v}$ contained in $S_{0}$, which has the property that for each $\alpha \in Q_{W}$, and each $\theta \in S_{1} \cup \ldots \cup$ $S_{T} \cup S_{1}^{\#} \cup \ldots \cup S_{T}^{\#}$,

$$
\operatorname{ord}_{v}(\alpha-\theta)<A_{0}+\log _{v}(n) .
$$

For large enough $n$, the existence of such a subsequence follows from Lemma 8.6(B), applied to the regular sequence $S_{0} \cup S_{1} \cup \ldots \cup S_{T}$. Write

$$
Q_{W}=\left\{\alpha_{i_{0}}^{(T)}, \ldots, \alpha_{i_{W-1}}^{(T)}\right\}
$$

listing the elements in their order as a regular sequence. 
To patch $u_{v}^{(k-1)}(z)$ to $u_{v}^{(k)}(z)$, put

$$
f_{k}(z)=\prod_{l=0}^{k-1}\left(z-\alpha_{i_{l}}^{(k-1)}\right), \quad w_{v}^{(k)}(z)=u_{v}^{(k-1)}(z) / f_{k}(z)
$$

(using the same indices as in $Q_{W}$ ) and set

$$
u_{v}^{(k)}(z)=u_{v}^{(k-1)}(z)+\Delta_{v}^{(k)} w_{v}^{(k)}(z)=\left(f_{k}(z)+\Delta_{v}^{(k)}\right) w_{v}^{(k)}(z) .
$$

Since $\operatorname{ord}_{v}\left(\Delta_{v}^{(k)}\right) \geq k \log _{v}\left(h_{v}\right) \geq k V\left(E_{v}\right)+A_{2}+A_{0}+\left\lceil\log _{v}(n)\right\rceil$, Lemma 8.8 shows that the roots $\alpha_{i_{l}}^{(k)}:=\left(\alpha_{i_{l}}^{(k-1)}\right)^{*}$ of $f_{k}^{*}(z)=f_{k}(z)+\Delta_{v}^{(k)}$ satisfy

$$
\operatorname{ord}_{v}\left(\alpha_{i_{l}}^{(k)}-\alpha_{i_{l}}^{(k-1)}\right) \geq A_{0}+\left\lceil\log _{v}(n)\right\rceil,
$$

so they retain their positions in a regular sequence of length $n$ and do not move closer to the roots in $S_{1}^{\#}, \ldots, S_{T}^{\#}$; moreover $\left\{\alpha_{i_{0}}^{(l)}, \ldots, \alpha_{i_{W-1}}^{(l)}\right\}$ continues to form a regular sequence of length $W$ in $E_{v}$. The remaining roots of $u_{v}^{(l)}(z)$ are the same as those of $u_{v}^{(l-1)}(z)$.

Thus, we can patch the coefficients $c_{v, k}^{(k)}, k=T+1, \ldots, W$.

PHASE 5. Completing the patching process $(W+1 \leq k \leq n)$. To patch the coefficients $c_{v, k}^{(k)}$ for $k=W+1, \ldots, n$, we apply Lemma 8.10 as indicated above, with $Z=T$ and $M=A_{4} \log _{v}(n)$, taking $\Theta$ to be the set of roots of $u_{v}^{(k-1)}(z)$, and taking the $\Theta_{l}$ for $l=1, \ldots, T$ to be the sets of roots obtained from $S_{1}^{\#}, \ldots, S_{T}^{\#}$ by the patching process (at the step $k=W+1$ they are exactly the $\left.S_{l}^{\#}\right)$. Put

$$
f_{k}(z)=\prod_{l=0}^{k-1}\left(z-\alpha_{l}^{(k-1)}\right), \quad w_{v}^{(k)}(z)=u_{v}^{(k-1)}(z) / f_{k}(z)
$$

and

$$
u_{v}^{(k)}(z)=u_{v}^{(k-1)}(z)+\Delta_{v}^{(k)} w_{v}^{(k)}(z)=\left(f_{k}(z)+\Delta_{v}^{(k)}\right) w_{v}^{(k)}(z) .
$$

By the choice of $W$ in (8.39), we have

$$
\operatorname{ord}_{v}\left(\Delta_{v}^{(k)}\right) \geq k \log _{v}\left(h_{v}\right) \geq k V\left(E_{v}\right)+A_{2}+T \cdot\left(M+\log _{v}(R)\right)+M
$$

By Lemma 8.10, the roots $\alpha_{l}^{(k)}:=\left(\alpha_{l}^{(k-1)}\right)^{*}$ of $f_{k}^{*}(z)=f_{k}(z)+\Delta_{v}^{(k)}$ satisfy

$$
\operatorname{ord}_{v}\left(\alpha_{l}^{(k)}-\alpha_{l}^{(k-1)}\right) \geq A_{4} \log _{v}(n) \quad \text { for } l=0, \ldots, k-1
$$

while the remaining roots of $u_{v}^{(k)}(z)$ are the same as those of $u_{v}^{(k-1)}(z)$.

Thus the hypotheses of Lemma 8.10 continue hold, and the process carries through to the end. 
9. Local patching for irreducibility and ramification. In this section we will present two simple propositions which show that at a nonarchimedean place where $E_{v}$ is the trivial set $\widehat{O}_{v}$, patching can be carried out in such a way that the final polynomial is irreducible over $K_{v}$, and the extension $L_{w} / K_{v}$ obtained by adjoining one of its roots is either unramified, or totally ramified.

Let $O_{v}$ be the ring of integers of $K_{v}$, let $\mathfrak{m}_{v}$ be the maximal ideal of $O_{v}$, let $\pi_{v} \in \mathfrak{m}_{v}$ be a uniformizing parameter, and let $k_{v}=O_{v} / \mathfrak{m}_{v}$ be the residue field.

Proposition 9.1. Suppose $K_{v}$ is nonarchimedean, and $E_{v}=\widehat{O}_{v}$. Let $0<h_{v}<r_{v}<\gamma\left(E_{v}\right)=1$ be given, let $L$ be any positive integer, and suppose $0<B_{v}<1$. For each $n$ there is a monic polynomial $u_{v}^{(0)}(z) \in O_{v}[z]$ of degree $n$, whose roots belong to $E_{v}$ and which can be patched with arbitrary $\Delta_{v}^{(k)} \in K_{v}$ satisfying

$$
\left|\Delta_{v}^{(k)}\right|_{v} \leq \begin{cases}B_{v} & \text { for } k \leq L, \\ h_{v}^{k} & \text { for } k>L,\end{cases}
$$

in such a way that its roots remain in $E_{v}$, and $u_{v}^{(n)}(z)\left(\bmod \mathfrak{m}_{v}\right)$ is irreducible over $k_{v}[z]$. In particular, $u_{v}^{(n)}(z)$ is irreducible over $K_{v}[z]$, and for any root $\alpha$ of $u_{v}^{(n)}(z)$, if $L_{w}=K_{v}(\alpha)$ then $L_{w} / K_{v}$ is unramified of degree $n$.

Proof. Let $\bar{u}(z) \in k_{v}[z]$ be any monic irreducible polynomial of degree $n$, and let $u_{v}^{(0)}(z) \in O_{v}[z]$ be a monic polynomial of degree $n$ such that

$$
u_{v}^{(0)}(z)\left(\bmod \mathfrak{m}_{v}\right)=\bar{u}(z) .
$$

Patch $u_{v}^{(0)}(z)$ successively to $u_{v}^{(1)}(z), u_{v}^{(2)}(z), \ldots, u_{v}^{(n)}(z)$ by setting $u_{v}^{(k)}(z)=$ $u_{v}^{(k-1)}(z)+\Delta_{v}^{(k)} z^{n-k}$. As each $\left|\Delta_{v}^{(k)}\right|_{v}<1$ for each $k$, at each step we have

$$
u_{v}^{(k)}(z)\left(\bmod \mathfrak{m}_{v}\right)=\bar{u}(z)
$$

Proposition 9.2. Suppose $K_{v}$ is nonarchimedean, and $E_{v}=\widehat{O}_{v}$. Let $0<h_{v}<r_{v}<\gamma\left(E_{v}\right)=1$ be given, let $L$ be any positive integer, and suppose $0<B_{v}<1$. Let $u_{v}^{(0)}(z) \in O_{v}[z]$ be an Eisenstein polynomial of degree $n$. If $n$ is sufficiently large, then $u_{v}^{(0)}(z)$ can be patched with arbitrary $\Delta_{v}^{(k)} \in K_{v}$ satisfying

$$
\left|\Delta_{v}^{(k)}\right|_{v} \leq \begin{cases}B_{v} & \text { for } k \leq L, \\ h_{v}^{k} & \text { for } k>L,\end{cases}
$$

in such a way $u_{v}^{(n)}(z)$ remains an Eisenstein polynomial. In particular, $u_{v}^{(n)}(z)$ is irreducible over $K_{v}[z]$ and has all its roots in $E_{v}$, and for any root $\alpha$ of $u_{v}^{(n)}(z)$, if $L_{w}=K_{v}(\alpha)$ then $L_{w} / K_{v}$ is totally ramified of degree $n$. 
Proof. Let $u_{v}^{(0)}(z) \in O_{v}[z]$ be an Eisenstein polynomial of degree $n$. Patch $u_{v}^{(0)}(z)$ successively to $u_{v}^{(1)}(z), u_{v}^{(2)}(z), \ldots, u_{v}^{(n)}(z)$ by setting

$$
u_{v}^{(k)}(z)=u_{v}^{(k-1)}(z)+\Delta_{v}^{(k)} z^{n-k} .
$$

By hypothesis $\left|\Delta_{v}^{(k)}\right|_{v}<1$ at each step; if $n>L$ is large enough that $h_{v}^{n}<\left|\pi_{v}\right|_{v}$, then $\left|\Delta_{v}^{(n)}\right|_{v} \leq\left|\pi_{v}^{2}\right|_{v}$ and $u_{v}^{(n)}(z)$ will also be an Eisenstein polynomial.

10. The global patching argument. We can now complete the proof of Theorem 2.3. For this, we will need the following version of the Strong Approximation Theorem, which can easily be derived from the Lemma on p. 66 of [4]. Let $\mathcal{S}$ be a finite set of places of $K$ including all the archimedean places. Recall that the set of $\mathcal{S}$-integers of $K$ is

$$
K^{\mathcal{S}}:=\left\{\kappa \in K:|\kappa|_{v} \leq 1 \text { for all } v \notin \mathcal{S}\right\} .
$$

Lemma 10.1. Let $K$ be a number field, and let $\mathcal{S}$ be a finite set of places of $K$ containing all the archimedean places. There is a constant $C(K, \mathcal{S})$, depending only on $K$ and $\mathcal{S}$, such that if numbers $C_{v}>0$ are specified for each $v \in \mathcal{S}$, subject to the condition

$$
\prod_{v \in \mathcal{S}} C_{v}^{D_{v}}>C(K, \mathcal{S})
$$

then for any elements $c_{v} \in K_{v}(v \in \mathcal{S})$ there is an $\mathcal{S}$-integer c with $\left|c-c_{v}\right|_{v} \leq$ $C_{v}$ for all $v \in \mathcal{S}$.

REMARK. If each $C_{v}$ were required to belong to the value group of $K_{v}^{\times}$, then the constant $C(K, \mathcal{S})$ would depend only on $K$.

Proof of Theorem 2.3. Let $\widehat{S}$ be the finite set of places of $K$ consisting of all archimedean $v$ and all nonarchimedean $v$ where $E_{v} \neq \widehat{O}_{v}$. By the hypotheses of Theorem 2.3 and the preliminary reductions (1)-(5), we can assume that $\gamma(\mathbb{E})>1$, that each $E_{v}$ and $U_{v}$ is stable under $\operatorname{Gal}_{c}\left(\mathbb{C}_{v} / K_{v}\right)$, and

(1) For each archimedean $v, E_{v}$ is compact;

(2) For each nonarchimedean $v \in S, E_{v}=\bigcup_{i=1}^{M} a_{i}+\pi_{v}^{n_{i}} O_{v}$;

(3) For each nonarchimedean $v \in \widehat{S} \backslash S, E_{v}$ is a PL-domain;

(4) For each $v \notin \widehat{S}, E_{v}=\widehat{O}_{v}$.

We are also given a finite set of places $S^{\prime}=S_{1}^{\prime} \cup S_{2}^{\prime}$ of $K$, disjoint from $\widehat{S}$, such that each $v \in S_{1}^{\prime}$ is to be inert, and each $v \in S_{2}^{\prime}$ is to be totally ramified, in the extensions $K(\alpha) / K$. We can assume that $S^{\prime}$ is nonempty. 
Using $\prod_{v \in \widehat{S} \cup S^{\prime}} \gamma\left(E_{v}\right)^{D_{v}}=\gamma(\mathbb{E})>1$, fix numbers $0<h_{v}<r_{v}<\gamma\left(E_{v}\right)$ for each $v \in \widehat{S} \cup S^{\prime}$, with

$$
H:=\prod_{v \in \widehat{S} \cup S^{\prime}} h_{v}^{D_{v}}>1
$$

For each $v \in \widehat{S}$, let $M_{v}$ be the constant from the corresponding local patching proposition $5.1,6.1,7.1$, or 8.1 .

Fix an integer $L>0$ subject to the appropriate local condition (5.1), (6.1), (7.1), or (8.1) for each $v \in \widehat{S}$ : namely

$$
\begin{cases}\left(\frac{h_{v}}{r_{v}}\right)^{L} \frac{M_{v}}{1-h_{v} / r_{v}}<\frac{1}{8} & \text { if } K_{v} \cong \mathbb{C}, \text { or if } K_{v} \cong \mathbb{R} \text { but } E_{v} \varsubsetneqq \mathbb{R}, \\ \left(\frac{h_{v}}{r_{v}}\right)^{L} \frac{M_{v}}{1-h_{v} / r_{v}}<\frac{1}{16} \text { if } K_{v} \cong \mathbb{R} \text { and } E_{v} \subset \mathbb{R} & \text { if } K_{v} \text { is nonarchimedean and } E_{v} \text { is a PL-domain, } \\ \left(\frac{h_{v}}{r_{v}}\right)^{L} M_{v} \leq \frac{1}{2} & \text { if } K_{v} \text { is nonarchimedean and } E_{v} \subset K_{v} \\ L \cdot\left(\frac{h_{v}}{r_{v}}\right)^{L} M_{v} \leq 1\end{cases}
$$

and also subject to the global condition

$$
H^{L}>C\left(K, \widehat{S} \cup S^{\prime}\right)
$$

where $C\left(K, \widehat{S} \cup S^{\prime}\right)$ is the constant from Lemma 10.1 above.

Next choose constants $B_{v}$, for $v \in \widehat{S} \cup S^{\prime}$, as follows. For each nonarchimedean $v \in \widehat{S}$, let $B_{v}$ be the constant specified in Proposition 7.1 or 8.1; note that $B_{v}$ depends on $L$. For each $v \in S^{\prime}$, take $B_{v}=1 / 2$. Then, recalling that in Propositions 5.1 and 6.1 the constants $B_{v}$ can be specified arbitrarily, choose numbers $B_{v}>0$ for the archimedean $v \in \widehat{S}$ in such a way that

$$
\prod_{v \in \widehat{S} \cup S^{\prime}} B_{v}^{D_{v}}>C\left(K, \widehat{S} \cup S^{\prime}\right) .
$$

Finally, let $n \geq L$ be any integer sufficiently large that for each $v \in$ $\widehat{S} \cup S^{\prime}$ the appropriate local patching proposition $5.1,6.1,7.1,8.1,9.1$, or 9.2 applies, relative to the given $E_{v}, U_{v}, L$, and $B_{v}$.

For each $v \in \widehat{S} \cup S^{\prime}$, let $u_{v}^{(0)}(z) \in K_{v}[z]$ be the monic polynomial of degree $n$ given by the corresponding local patching proposition. We will patch these to a common global polynomial $u(z) \in K[z]$. Suppose inductively there are global $\widehat{S} \cup S^{\prime}$-integers $c_{l} \in K$, for $l=1, \ldots, k-1$, such that the coefficients of the polynomials

$$
u_{v}^{(k-1)}(z)=z^{n}+\sum_{l=1}^{n} c_{v, l}^{(k-1)} z^{n-l}
$$


satisfy

$$
c_{v, l}^{(k-1)}=c_{l}
$$

for all $v \in \widehat{S} \cup S^{\prime}$ and all $l=1, \ldots, k-1$. If $k \leq L,(10.2)$ together with Proposition 10.1 shows that there is a global $\widehat{S} \cup S^{\prime}$-integer $c_{k}$ satisfying

$$
\left|c_{k}-c_{v, k}^{(k-1)}\right|_{v} \leq B_{v}
$$

for each $v$. If $k>L$, then by (10.1),

$$
\prod_{v \in S}\left(h_{v}^{k}\right)^{D_{v}}=H^{k}>C\left(K, \widehat{S} \cup S^{\prime}\right)
$$

and Proposition 10.1 shows that there is a global $\widehat{S} \cup S^{\prime}$-integer $c_{k}$ such that

$$
\left|c_{k}-c_{v, k}^{(k-1)}\right|_{v} \leq h_{v}^{k}
$$

for each $v$. In either case, put $\Delta_{v}^{(k)}=c_{k}-c_{v, k}^{(k-1)}$ for each $v$, and patch $u_{v}^{(k-1)}(z)$ to $u_{v}^{(k)}(z)$ using the appropriate local patching construction.

At the end of the patching process, we have a global polynomial

$$
u(z)=z^{n}+\sum_{k=1}^{n} c_{k} z^{n-k}
$$

with $\widehat{S} \cup S^{\prime}$-integer coefficients such that $u(z)=u_{v}^{(n)}(z)$ for each $v \in \widehat{S} \cup S^{\prime}$. By the local patching constructions, the roots of $u(z)$ belong to $U_{v}$ for each $v \in$ $\widehat{S}$, and to $K_{v}$ for each $v \in S$. For each $v \in S_{1}^{\prime}, u(z)\left(\bmod \mathfrak{m}_{v}\right)$ is irreducible, and for each $v \in S_{2}^{\prime}, u(z)$ is an Eisenstein polynomial. In particular, $u(z)$ is irreducible; and if $\alpha \in \widetilde{K}$ is any root of $u(z)$, then each $v \in S_{1}^{\prime}$ is inert in $K(\alpha) / K$, and each $v \in S_{2}^{\prime}$ is totally ramified. Finally, because the $c_{k}$ are $S$-integers, the roots of $u(z)$ belong to $U_{v}=\widehat{O}_{v}$ for each $v \notin \widehat{S}$. This completes the proof.

11. Appendix I. Inner capacities of archimedean sets. In this appendix we will prove the following lemma, which is presumably well known but seems not to appear in the literature.

LEMMA 11.1. Let $E \subset \mathbb{C}$ be a compact set which is the closure of its interior $E^{0}$, and whose boundary is a finite union of smooth arcs. Then

$$
\gamma\left(E^{0}\right)=\gamma(E)
$$

Proof. Put $F_{0}=\emptyset$. For each $n \geq 1$, let $F_{n} \subset E^{0}$ be a compact set containing $F_{n-1}$ and all $z \in E$ with distance at least $1 / n$ from the boundary $\partial E$, so $\bigcup_{n=1}^{\infty} F_{n}=E^{0}$. The potential function and Robin constant of $F_{n}$ are

$$
u_{n}(z)=u\left(z, F_{n}\right)=\int-\log (|z-w|) d \mu_{n}(w),
$$




$$
V\left(F_{n}\right)=\iint-\log (|z-w|) d \mu_{n}(z) d \mu_{n}(w) .
$$

Let $u(z)$ be the potential function, and $V(E)$ the Robin constant, of $E$. Put

$$
\widehat{V}(E)=\lim _{n \rightarrow \infty} V\left(F_{n}\right) .
$$

The limit is decreasing, since the $F_{n}$ are increasing. We will prove that $\widehat{V}(E)=V(E)$, so

$$
\lim _{n \rightarrow \infty} \gamma\left(F_{n}\right)=\gamma(E)
$$

Let $\mu_{n}$ be the equilibrium distribution of $F_{n}$. Our strategy is to show that the sequence $\left\{\mu_{n}\right\}$ converges weakly to the equilibrium distribution $\mu$ of $E$. In any case, after passing to a subsequence of the $F_{n}$, we can suppose that $\left\{\mu_{n}\right\}$ converges weakly to some measure $\widehat{\mu}$. Clearly $\widehat{\mu}$ is a probability measure supported on $\partial E$. We will now study the properties of the potential function

$$
\widehat{u}(z)=\int-\log (|z-w|) d \widehat{\mu}(w)
$$

associated to $\widehat{\mu}$.

STEP 1. We claim that $\widehat{u}(z)=\widehat{V}(E)$ for all $z \in E^{0}$. To see this, fix $z_{0} \in E^{0}$, and let $\delta=\inf _{x \in \partial E}\left(\left|z_{0}-x\right|\right)$ be the distance from $z$ to $\partial E$. Let $N$ be large enough that $1 / N<\delta$. Then for all $n \geq N, z_{0}$ is in the interior of $F_{n}$, and hence $u_{n}\left(z_{0}\right)=V\left(F_{n}\right)$. The function $\log \left(\left|z_{0}-w\right|\right)$ is continuous and bounded on the set

$$
\partial E(1 / N):=\{z \in \mathbb{C}:|z-x|<1 / N \text { for some } x \in \partial E\},
$$

so by weak convergence,

$$
\begin{aligned}
\widehat{u}\left(z_{0}\right) & =\int-\log \left(\left|z_{0}-w\right|\right) d \widehat{\mu}(w)=\lim _{n \rightarrow \infty} \int-\log \left(\left|z_{0}-w\right|\right) d \mu_{n}(w) \\
& =\lim _{n \rightarrow \infty} u_{n}\left(z_{0}\right)=\lim _{n \rightarrow \infty} V\left(F_{n}\right)=\widehat{V}(E) .
\end{aligned}
$$

STEP 2. We claim that $\widehat{u}(z) \leq \widehat{V}(E)$ for all $z \in E$. Indeed, for any $M$ and $n$,

$$
\begin{aligned}
u_{n}^{(M)}(z) & :=\int \min (M,-\log (|z-w|)) d \mu_{n}(w) \\
& \leq \int-\log (|z-w|) d \mu_{n}(w)=u_{n}(z) \leq V\left(F_{n}\right),
\end{aligned}
$$

and so by weak convergence

$$
\begin{aligned}
\widehat{u}^{(M)}(z) & =\int \min (M,-\log (|z-w|)) d \widehat{\mu}(w) \\
& =\lim _{n \rightarrow \infty} u_{n}^{(M)}(z) \leq \lim _{n \rightarrow \infty} V\left(F_{n}\right)=\widehat{V}(E) .
\end{aligned}
$$

Hence, letting $M \rightarrow \infty$, we obtain

$$
\widehat{u}(z)=\lim _{M \rightarrow \infty} \widehat{u}^{(M)}(z) \leq \widehat{V}(E) .
$$


STEP 3. Third, we claim that $\widehat{u}(z)=\widehat{V}(E)$ for all $z \in E$ except possibly a finite set of points. Here we use the hypotheses that $\partial E$ is a finite union of smooth arcs, and that $E$ is the closure of $E^{0}$. Take any point $z_{0} \in \partial E$ which is not an intersection point of two arcs. Then there is a well-defined normal line $\mathcal{N}$ to $\partial E$ at $z_{0}$. For small enough $r$, the ball $B\left(z_{0}, r\right)$ will not meet any of the arcs comprising $\partial E$ except for the one containing $z_{0}$. Also for small enough $r$, if $w \in \partial E \cap B\left(z_{0}, r\right)$ with $w \neq z_{0}$, and $x \in \mathcal{N} \cap E^{0} \cap B\left(z_{0}, r\right)$ then the angle between the segments $\overline{z_{0} x}$ and $\overline{z_{0} w}$ will be at least $\pi / 4$. Fix an $r$ satisfying these two conditions. We assert that there is a constant $C$ such that for all $x \in \mathcal{N} \cap E^{0} \cap B\left(z_{0}, r / 2\right)$, and all $w \in \partial E$,

$$
-\log (|x-w|) \leq-\log \left(\left|z_{0}-w\right|\right)+C .
$$

For $w \in \partial E \backslash B\left(z_{0}, r / 2\right)$, and $x \in \mathcal{N} \cap E^{0} \cap B\left(z_{0}, r\right)$, both $-\log (|x-w|)$ and $-\log \left(\left|z_{0}-w\right|\right)$ are uniformly bounded, so the assertion is trivial. For any fixed $w \in \partial E \cap B\left(z_{0}, r\right)$ with $w \neq z_{0}$, if $x_{0} \in \mathcal{N} \cap E \cap B\left(z_{0}, r\right)$ is the point nearest to $z$, then by our hypothesis we have $\left|x_{0}-w\right| \geq\left|z_{0}-w\right| \sin (\pi / 4)$, and hence for any $x \in \mathcal{N} \cap E^{0} \cap B\left(z_{0}, r\right)$,

$$
-\log (|x-w|) \leq-\log \left(\left|x_{0}-w\right|\right) \leq-\log \left(\left|z_{0}-w\right|\right)+\log (\sqrt{2}) .
$$

Finally, if $w=z_{0}$ the assertion is again trivial.

By Step 2,

$$
\int\left(-\log \left(\left|z_{0}-w\right|\right)+C\right) d \widehat{\mu}(w) \leq \widehat{V}(E)+C<\infty .
$$

Let $x_{1}, x_{2}, \ldots$ be a sequence of points in $\mathcal{N} \cap E^{0} \cap B\left(z_{0}, r\right)$ with $\lim _{n \rightarrow \infty} x_{n}$ $=z_{0}$. Then pointwise, for each $z \in \partial E, \lim _{n \rightarrow \infty}\left|z-x_{n}\right|=\left|z-z_{0}\right|$. By Lebesgue's dominated convergence theorem, (11.1) and Step 1,

$$
\begin{aligned}
\widehat{u}\left(z_{0}\right) & :=\int-\log \left(\left|z_{0}-w\right|\right) d \widehat{\mu}(w)=\lim _{n \rightarrow \infty} \int-\log \left(\left|x_{n}-w\right|\right) d \widehat{\mu}(w) \\
& =\lim _{n \rightarrow \infty} \widehat{u}\left(x_{n}\right)=\widehat{V}(E) .
\end{aligned}
$$

Thus, $\widehat{u}(z)=\widehat{V}(E)$ for all $z \in E$, except possibly on set of capacity 0 (the finite set of intersection points of the arcs in $\partial E$ ).

STEP 4. We can now show that $V(E)=\widehat{V}(E)$ and $\widehat{\mu}=\mu$, the equilibrium distribution of $E$. Then since $u_{E}(z)=V(E)$ for all $z \in E$ except possibly on a set of capacity 0 , which has $\mu$-measure 0 , and $\widehat{u}(z)=\widehat{V}(E)$ for all $z \in E$ except possibly on a set of capacity 0 , which has $\widehat{\mu}$-measure 0 (see [13, Theorem III.7, p. 56]), Fubini's theorem gives

$$
\begin{aligned}
V(E) & =\int u_{E}(z) d \mu(z)=\iint-\log (|z-w|) d \mu(z) d \widehat{\mu}(w) \\
& =\int \widehat{u}(w) d \widehat{\mu}(z)=\widehat{V}(E) .
\end{aligned}
$$


Furthermore,

$$
\iint-\log (|z-w|) d \widehat{\mu}(z) d \widehat{\mu}(w)=\int \widehat{u}(w) d \widehat{\mu}(z)=V(E) .
$$

This is the defining property of $\mu$, and $\mu$ is unique. Hence $\widehat{\mu}=\mu$.

12. Appendix II. Proof of a lemma of Cantor. In this section we will give a proof of the key fact needed for the local patching construction for nonarchimedean PL-domains, the fact that finite unions of balls are PL-domains. The proof follows an argument sketched by Cantor ([3, Lemma 3.2.3]): Cantor's proof, though basically correct, was incomplete.

We remark that the result in now known in a much more general setting, for algebraic curves (see [11, Theorem 4.2.12, p. 244]). It has also been extended for the affine line over a valuation field with a valuation group of arbitrary rank (see [7]). Related results have been proved by Fieseler and by Matignon in the context of rigid analysis. However, the argument given here leads to an explicit construction of the polynomial, which is not true in the general case.

Let $v$ be a nonarchimedean place of $K$. Suppose $a \in \mathbb{C}_{v}$, and $r>0$. Recall that we write

$$
\begin{aligned}
B(a, r) & =\left\{z \in \mathbb{C}_{v}:|z-a|_{v} \leq r\right\}, \\
\partial B(a, r) & =\left\{z \in \mathbb{C}_{v}:|z-a|_{v}=r\right\}, \\
B(a, r)^{-} & =\left\{z \in \mathbb{C}_{v}:|z-a|_{v}<r\right\} .
\end{aligned}
$$

By a finite union of balls, we mean a set of the form

$$
E_{v}=\bigcup_{i=1}^{M} B\left(a_{i}, r_{i}\right)
$$

where each $r_{i}$ belongs to the value group of $\mathbb{C}_{v}^{\times}$. By the ultrametric inequality, we can assume the $B\left(a_{i}, r_{i}\right)$ are pairwise disjoint. Fix such a set $E_{v}$.

Let $\zeta$ be a point in $\mathbb{P}^{1}\left(\mathbb{C}_{v}\right) \backslash E_{v}$. Our goal is to show that there is a rational function $f(z)$, whose only poles are at $\zeta$, and whose zeros are supported on the $a_{i}$ 's, such that

$$
E_{v}=\left\{z \in \mathbb{P}^{1}\left(\mathbb{C}_{v}\right):|f(z)|_{v} \leq 1\right\} .
$$

To motivate the construction, first suppose that $\zeta=\infty$, and that $f(z)$ could be taken to be polynomial of the form

$$
f(z)=\pi_{v}^{n w_{0}} \prod_{i=1}^{M}\left(z-a_{i}\right)^{n w_{i}}
$$

where $n$ is the degree of $f(z)$, and the $w_{i}$ are rational numbers with $\sum_{i=1}^{M} w_{i}$ $=1$ such that $w_{i} \geq 0$ for $i=1, \ldots, M$, and $\pi_{v}$ is a uniformizing parameter 
for $K_{v}$. Write

$$
\Theta_{i j}= \begin{cases}\log _{v}\left(\left|a_{i}-a_{j}\right|_{v}\right) & \text { if } i \neq j, \\ \log _{v}\left(r_{i}\right) & \text { if } i=j,\end{cases}
$$

noting that since the balls $B\left(a_{i}, r_{i}\right)$ are disjoint we have $\Theta_{i j}>\Theta_{i i}$ if $i \neq j$.

The numbers $w_{i}$ are subject to several constraints. To see them, take $x \in$ $\partial B\left(a_{i}, r_{i}\right)$. Then $\log _{v}\left(\left|x-a_{j}\right|_{v}\right)=\Theta_{i j}$ for $j=1, \ldots, M$, and by hypothesis $\log _{v}\left(|f(x)|_{v}\right)=0$. This leads to the system of equations

$$
0=w_{0}+\sum_{j=1}^{M} \Theta_{i j} w_{j} \quad \text { for } i=1, \ldots, M .
$$

In addition we have the relation

$$
1=0 \cdot w_{0}+\sum_{j=1}^{M} 1 \cdot w_{j}
$$

If we write

$$
\left\{\begin{array}{l}
\Theta_{00}=0, \\
\Theta_{0 j}=1 \text { for } j=1, \ldots, M, \\
\Theta_{i 0}=1 \text { for } i=1, \ldots, M,
\end{array}\right.
$$

and put $\Theta=\left(\Theta_{i j}\right)_{0 \leq i, j \leq M}$, then the system of equations (12.2), (12.3) becomes

$$
\left[\begin{array}{c}
1 \\
0 \\
\vdots \\
0
\end{array}\right]=\Theta\left[\begin{array}{c}
w_{0} \\
w_{1} \\
\vdots \\
w_{m}
\end{array}\right]
$$

Next, suppose $\zeta \neq \infty$. In this case one could hope $f(z)$ would have the form

$$
f(z)=\pi_{v}^{n w_{0}} \cdot \frac{\prod_{i=1}^{M}\left(z-a_{i}\right)^{n w_{i}}}{(z-\zeta)^{n}} .
$$

Taking $x \in \partial B\left(a_{i}, r_{i}\right)$ and using $|f(x)|_{v}=1$, we find

$$
0=n w_{0}+\left(\sum_{j=1}^{M} \log _{v}\left(\left|x-a_{i}\right|_{v}\right) n w_{j}\right)-n \log _{v}\left(|x-\zeta|_{v}\right)
$$

which, since $|x-\zeta|_{v}=\left|\zeta-a_{i}\right|_{v}$, can be written as

$$
\log _{v}\left(\left|\zeta-a_{i}\right|_{v}\right)=w_{0}+\sum_{j=1}^{M} \log _{v}\left(\left|x-a_{i}\right|_{v}\right) w_{j}
$$


for each $i$. Thus we are led to the system of equations

$$
\left[\begin{array}{c}
1 \\
\log _{v}\left(\left|\zeta-a_{1}\right|_{v}\right) \\
\vdots \\
\log _{v}\left(\left|\zeta-a_{M}\right|_{v}\right)
\end{array}\right]=\Theta\left[\begin{array}{c}
w_{0} \\
w_{1} \\
\vdots \\
w_{M}
\end{array}\right]
$$

Cantor's idea was to reverse this construction: if the matrix $\Theta$ could be shown to be nonsingular, then the $w_{i}$ 's would be uniquely defined by the equations above; and if they could be shown to be positive, then it would be natural to expect that they could be used to construct a function $f(z)$ with the desired property.

Write $\mathbf{1}_{M}$ for the $M$-element column vector whose entries are all 1's, and write $\mathbf{J}_{M}$ for the $M \times M$ matrix whose entries are all 1's. For any matrix $A$, write ${ }^{\mathrm{t}} A$ for its transpose. Observe that

$$
\mathbf{J}_{M}=\mathbf{1}_{M}{ }^{\mathrm{t}} \mathbf{1}_{M} .
$$

Further, write $\Theta^{\prime}=\left(\Theta_{i j}\right)_{1 \leq i, j \leq M}$, so that in terms of block matrices,

$$
\Theta=\left[\begin{array}{cc}
0 & { }^{\mathrm{t}} \mathbf{1}_{M} \\
\mathbf{1}_{M} & \Theta^{\prime}
\end{array}\right] .
$$

Cantor's Lemma 3.2.3 can be formulated as follows:

LEMma 12.1. The matrix $\Theta$ is nonsingular, and if $w_{0}, w_{1}, \ldots, w_{m}$ are defined by

$$
\left[\begin{array}{c}
w_{0} \\
w_{1} \\
\vdots \\
w_{M}
\end{array}\right]=\Theta^{-1}\left[\begin{array}{c}
1 \\
0 \\
\vdots \\
0
\end{array}\right], \quad r e s p . \quad\left[\begin{array}{c}
w_{0} \\
w_{1} \\
\vdots \\
w_{M}
\end{array}\right]=\Theta^{-1}\left[\begin{array}{c}
1 \\
\log _{v}\left(\left|\zeta-a_{1}\right|_{v}\right) \\
\vdots \\
\log _{v}\left(\left|\zeta-a_{M}\right|_{v}\right)
\end{array}\right]
$$

according as $\zeta=\infty$ or $\zeta \neq \infty$, then $w_{1}, \ldots, w_{m}>0$ and $\sum_{i=1}^{m} w_{i}=1$; and if $f(z)$ is defined by (12.1), respectively (12.4), then

$$
E_{v}=\left\{z \in \mathbb{P}^{1}\left(\mathbb{C}_{v}\right):|f(z)|_{v} \leq 1\right\} .
$$

Furthermore, we have the following formula for $\Theta^{-1}$ : let $\delta$ be any number satisfying $\delta>\max _{1 \leq i, j \leq m}\left(\Theta_{i j}\right)$. Then

(A) the symmetric matrix $\mathbf{A}_{\delta}:=\Theta^{\prime}-\delta \mathbf{J}_{M}$ is negative definite;

(B) if we define $\alpha$ by $\alpha^{-1}=-{ }^{\mathrm{t}} \mathbf{1}_{M} \mathbf{A}_{\delta}^{-1} \mathbf{1}_{M}$ and put $\mathbf{b}=-\mathbf{A}_{\delta}^{-1} \mathbf{1}_{M}$, then $\alpha>0$ and

$$
\Theta^{-1}=\left[\begin{array}{cc}
\alpha-\delta & \alpha^{\mathrm{t}} \mathbf{b} \\
\alpha \mathbf{b} & \mathbf{A}_{\delta}^{-1}+\alpha \mathbf{b}^{\mathrm{t}} \mathbf{b}
\end{array}\right] .
$$


Proof. We first prove (A) and (B), by induction on $M$. Fix $\delta$, and write $\mathbf{A}=\mathbf{A}_{\delta}$.

If $M=1$, then $\mathbf{A}=\Theta_{11}-\delta<0$, so (A) holds, and we find

$$
b=\frac{-1}{\Theta_{11}-\delta}, \quad \alpha=\frac{1}{b}=\delta-\Theta_{11}>0, \quad \Theta=\left[\begin{array}{cc}
0 & 1 \\
1 & \Theta_{11}
\end{array}\right] .
$$

The matrix on the right side of $(\mathrm{B})$ is

$$
\begin{aligned}
{\left[\begin{array}{cc}
\alpha-\delta & \alpha b \\
\alpha b & 1 / A+\alpha b^{2}
\end{array}\right] } & =\left[\begin{array}{cc}
-\Theta_{11} & 1 \\
1 & \frac{1}{\Theta_{11}-\delta}+\left(\delta-\Theta_{11}\right)\left(\frac{-1}{\Theta_{11}-\delta}\right)^{2}
\end{array}\right] \\
& =\left[\begin{array}{cc}
-\Theta_{11} & 1 \\
1 & 0
\end{array}\right]
\end{aligned}
$$

which equals $\Theta^{-1}$, so (B) holds as well.

Next take $M>1$ and suppose (A) and (B) have been proven for all $M^{\prime}<M$. Put

$$
\delta^{\prime}=\max _{1 \leq i, j \leq M} \Theta_{i j}
$$

and define an equivalence relation on $\left\{a_{1}, \ldots, a_{M}\right\}$ by $a_{i} \sim a_{j}$ if $\Theta_{i j}<\delta^{\prime}$. (To see this is an equivalence relation, note that since $M \geq 2$, for each $i$ there is a $j \neq i$, and $\Theta_{i i}<\Theta_{i j} \leq \delta^{\prime}$. This yields reflexivity. Symmetry is clear, and transitivity follows from the ultrametric inequality.) After relabeling the $a_{i}$ 's if necessary, we can assume that the equivalence classes consist of elements whose indices are consecutive blocks of integers. By the definition of $\delta^{\prime}$, there are at least two equivalence classes.

The matrix $\Theta^{\prime}$ thus has the form

$$
\left[\begin{array}{cccc}
\Theta_{1}^{\prime} & \delta^{\prime} & \ldots & \delta^{\prime} \\
\delta^{\prime} & \Theta_{2}^{\prime} & & \delta^{\prime} \\
\vdots & \vdots & \ddots & \vdots \\
\delta^{\prime} & \delta^{\prime} & & \Theta_{r}^{\prime}
\end{array}\right]
$$

with blocks corresponding to the various equivalence classes down the diagonal, and entries $\delta^{\prime}$ everywhere else. By the definition of the relation $\sim$, each entry in each $\Theta_{k}^{\prime}$ is strictly less than $\delta^{\prime}$. Consequently the matrix $\mathbf{A}^{\prime}:=\Theta^{\prime}-\delta^{\prime} \mathbf{J}_{M}$ has the block diagonal form $\operatorname{diag}\left(\mathbf{A}_{1}, \ldots, \mathbf{A}_{r}\right)$, where $\mathbf{A}_{k}=\Theta_{k}^{\prime}-\delta^{\prime} \mathbf{J}_{m_{k}}$ if $m_{k}$ is the number of elements of the $k$ th equivalence class. Since $\delta^{\prime}$ is strictly greater than every entry in $\Theta_{k}^{\prime}$, each $\mathbf{A}_{k}$ satisfies the hypotheses of the lemma, and by induction is negative definite. Therefore

$$
\mathbf{A}=\mathbf{A}^{\prime}-\left(\delta-\delta^{\prime}\right) \mathbf{J}_{M}
$$

is also negative definite, and in particular is nonsingular. This yields (A). 
To prove (B), write $\mathbf{A}=\mathbf{A}_{\delta}, \mathbf{1}=\mathbf{1}_{M}, \mathbf{J}=\mathbf{J}_{M}, \mathbf{b}=-\mathbf{A}^{-1} \mathbf{1}_{M}$, and let

$$
\nabla=\left[\begin{array}{cc}
\alpha-\delta & \alpha^{\mathrm{t}} \mathbf{b} \\
\alpha \mathbf{b} & \mathbf{A}^{-1}+\alpha \mathbf{b}^{\mathrm{t}} \mathbf{b}
\end{array}\right]
$$

note that $\alpha^{-1}=-{ }^{\mathrm{t}} \mathbf{1} \mathbf{A}^{-1} \mathbf{1}={ }^{\mathrm{t}} \mathbf{1} \mathbf{b}$. We compute

$$
\begin{aligned}
\Theta \cdot \nabla & =\left[\begin{array}{cc}
0 & { }^{\mathrm{t}} \mathbf{1} \\
\mathbf{1} & \Theta^{\prime}
\end{array}\right] \cdot\left[\begin{array}{cc}
\alpha-\delta & \alpha^{\mathrm{t}} \mathbf{b} \\
\alpha \mathbf{b} & \mathbf{A}^{-1}+\alpha \mathbf{b}^{\mathrm{t}} \mathbf{b}
\end{array}\right] \\
& =\left[\begin{array}{cc}
\alpha^{\mathrm{t}} \mathbf{1} \mathbf{b} & { }^{\mathrm{t}} \mathbf{1} \mathbf{A}^{-1}+\alpha^{\mathrm{t}} \mathbf{1} \mathbf{b}^{\mathrm{t}} \mathbf{b} \\
(\alpha-\delta) \mathbf{1}+\alpha \Theta^{\prime} \mathbf{b} & \alpha \mathbf{1}^{\mathrm{t}} \mathbf{b}+\Theta^{\prime}\left(\mathbf{A}^{-1}+\alpha \mathbf{b}^{\mathrm{t}} \mathbf{b}\right)
\end{array}\right] .
\end{aligned}
$$

Since $\mathbf{A}$ is negative definite, so is $\mathbf{A}^{-1}$, and consequently $\alpha>0$. Now let us compute the quantities in the four corners of $\Theta \cdot \nabla$. For the first three:

$$
\begin{aligned}
\alpha{ }^{\mathrm{t}} \mathbf{1} \mathbf{b} & =\alpha \cdot \alpha^{-1}=1 ; \\
{ }^{\mathrm{t}} \mathbf{1} \mathbf{A}^{-1}+\alpha^{\mathrm{t}} \mathbf{1} \mathbf{b}^{\mathrm{t}} \mathbf{b} & ={ }^{\mathrm{t}} \mathbf{1} \mathbf{A}^{-1}+\alpha{ }^{\mathrm{t}} \mathbf{1}\left(-\mathbf{A}^{-1} \mathbf{1}\right)\left(-{ }^{\mathrm{t}} \mathbf{1} \mathbf{A}^{-1}\right) \\
& ={ }^{\mathrm{t}} \mathbf{1} \mathbf{A}^{-1}+\alpha\left({ }^{\mathrm{t}} \mathbf{1} \mathbf{A}^{-1} \mathbf{1}\right)\left({ }^{\mathrm{t}} \mathbf{1} \mathbf{A}^{-1}\right) \\
& ={ }^{\mathrm{t}} \mathbf{1} \mathbf{A}^{-1}+\alpha \cdot\left(-\alpha^{-1}\right){ }^{\mathrm{t}} \mathbf{1} \mathbf{A}^{-1}=\mathbf{0} ; \\
(\alpha-\delta) \mathbf{1}+\alpha \Theta^{\prime} \mathbf{b} & =\alpha \mathbf{1}-\delta \mathbf{1}+\alpha \Theta^{\prime}\left(-\mathbf{A}^{-1} \mathbf{1}\right) \\
& =\alpha \mathbf{1}-\delta \mathbf{1}+\alpha(\mathbf{A}+\delta \mathbf{J})\left(-\mathbf{A}^{-1} \mathbf{1}\right) \\
& =\alpha \mathbf{1}-\delta \mathbf{1}-\alpha \mathbf{A} \mathbf{A}^{-1} \mathbf{1}-\delta \alpha \mathbf{1}^{\mathrm{t}} \mathbf{1} \mathbf{A}^{-1} \mathbf{1} \\
& =-\delta \mathbf{1}-(\delta \mathbf{1})\left(\alpha^{\mathrm{t}} \mathbf{1} \mathbf{A}^{-1} \mathbf{1}\right)=-\delta \mathbf{1}+\delta \mathbf{1}=\mathbf{0} .
\end{aligned}
$$

For the fourth, note first that

$$
\begin{aligned}
\alpha \mathbf{1} \mathbf{t}^{\mathrm{t}} \mathbf{b} & +\Theta^{\prime}\left(\mathbf{A}^{-1}+\alpha \mathbf{b}^{\mathrm{t}} \mathbf{b}\right) \\
& =\alpha \mathbf{1}\left({ }^{\mathrm{t}}\left(-\mathbf{A}^{-1} \mathbf{1}\right)\right)+(\mathbf{A}+\delta \mathbf{J})\left(\mathbf{A}^{-1}+\alpha\left(-\mathbf{A}^{-1} \mathbf{1}\right)^{\mathrm{t}}\left(-\mathbf{A}^{-1} \mathbf{1}\right)\right) \\
& =-\alpha \mathbf{1}^{\mathrm{t}} \mathbf{1} \mathbf{A}^{-1}+\mathbf{A} \mathbf{A}^{-1}+\delta \mathbf{J} \mathbf{A}^{-1}+\alpha \mathbf{A} \mathbf{A}^{-1} \mathbf{1}^{\mathrm{t}} \mathbf{1} \mathbf{A}^{-1}+\alpha \delta \mathbf{J} \mathbf{A}^{-1} \mathbf{1}^{\mathrm{t}} \mathbf{1} \mathbf{A}^{-1} \\
& =\mathbf{I}_{M}+\delta \mathbf{J} \mathbf{A}^{-1}+\alpha \delta \mathbf{J} \mathbf{A}^{-1} \mathbf{J} \mathbf{A}^{-1} .
\end{aligned}
$$

But, expanding $\mathbf{J}$ by columns and rows,

$$
\begin{aligned}
\mathbf{J A}^{-1} \mathbf{J A}^{-1} & =\mathbf{J}\left(\mathbf{A}^{-1} \mathbf{J}\right) \mathbf{A}^{-1} \\
& =\mathbf{J}\left[\mathbf{A}^{-1} \mathbf{1}, \ldots, \mathbf{A}^{-1} \mathbf{1}\right] \mathbf{A}^{-1}=\mathbf{J}[-\mathbf{b}, \ldots,-\mathbf{b}] \mathbf{A}^{-1} \\
& =-\left[\begin{array}{c}
{ }^{\mathrm{1}} \mathbf{1} \\
\vdots \\
{ }^{\mathrm{t}} \mathbf{1}
\end{array}\right][\mathbf{b}, \ldots, \mathbf{b}] \mathbf{A}^{-1}=-\left[\begin{array}{cc}
{ }^{\mathrm{t}} \mathbf{1} \mathbf{b} \ldots{ }^{\mathrm{t}} \mathbf{1} \mathbf{b} \\
\vdots & \vdots \\
{ }^{\mathrm{t}} \mathbf{1} \mathbf{b} \ldots{ }^{\mathrm{t}} \mathbf{1} \mathbf{b}
\end{array}\right] \mathbf{A}^{-1} \\
& =-\alpha^{-1} \mathbf{J} \mathbf{A}^{-1} .
\end{aligned}
$$

Consequently, $\delta \mathbf{J} \mathbf{A}^{-1}+\alpha \delta \mathbf{J} \mathbf{A}^{-1} \mathbf{J} \mathbf{A}^{-1}=\mathbf{0}$, so the lower right corner of $\Theta \cdot \nabla$ is the identity matrix $\mathbf{I}_{M}$. Thus $\Theta$ is invertible, and $\Theta^{-1}=\nabla$. This yields (B). 
So far we have followed Cantor's argument; now we leave it. We aim to prove the existence of a function $f(z)$ with poles only at $\zeta$ such that $E_{v}=\bigcup_{i=1}^{M} B\left(a_{i}, r_{i}\right)=\left\{z \in \mathbb{P}^{1}\left(\mathbb{C}_{v}\right):|f(z)|_{v} \leq 1\right\}$.

Suppose first that $\zeta=\infty$. We claim that if $w_{0}, w_{1}, \ldots, w_{M}$ are defined by

$$
\left[\begin{array}{c}
w_{0} \\
w_{1} \\
\vdots \\
w_{M}
\end{array}\right]=\Theta^{-1}\left[\begin{array}{c}
1 \\
0 \\
\vdots \\
0
\end{array}\right]
$$

then the $w_{i}$ are rational, $w_{1}, \ldots, w_{M}$ are positive, $\sum_{i=1}^{M} w_{i}=1$, and the polynomial $f(z)$ defined by (12.1) satisfies the required conditions (where we take the degree $n$ of $f(z)$ to be a number such that each $n w_{i}$ is integral).

The $w_{i}$ are rational since every element in $\Theta$ is. To prove the remaining assertions we proceed by induction on $M$.

If $M=1$, then

$$
\left[\begin{array}{l}
w_{0} \\
w_{1}
\end{array}\right]=\Theta^{-1}\left[\begin{array}{l}
1 \\
0
\end{array}\right]=\left[\begin{array}{cc}
-\Theta_{11} & 1 \\
1 & 0
\end{array}\right]\left[\begin{array}{l}
1 \\
0
\end{array}\right]=\left[\begin{array}{c}
-\Theta_{11} \\
1
\end{array}\right]
$$

where $w_{0}=\Theta_{11}=-\log _{v}\left(r_{1}\right)$. Thus $w_{1}=1>0$ and as a trivial sum $w_{1}=1$. Take $n$ so that $n w_{0}$ is integral. If we put

$$
f(z)=\pi_{v}^{n w_{0}}\left(z-a_{1}\right)^{n}
$$

then it is obvious that $B\left(a_{1}, r_{1}\right)=\left\{z \in \mathbb{P}^{1}\left(\mathbb{C}_{v}\right):|f(z)|_{v} \leq 1\right\}$.

Now take $M>1$ and suppose the assertions proved for $M^{\prime}<M$. Let the equivalence relation $\sim$ on $\left\{a_{1}, \ldots, a_{M}\right\}$ be as defined above, and keep the other notations established there. Suppose $\left\{a_{1}, \ldots, a_{m_{1}}\right\}$ is the first equivalence class. Put

$$
E_{v}^{\prime}=\bigcup_{i=1}^{m_{1}} B\left(a_{i}, r_{i}\right), \quad E_{v}^{\prime \prime}=\bigcup_{i=m_{1}+1}^{M} B\left(a_{i}, r_{i}\right)
$$

By induction, the numbers $w_{0}^{\prime}, w_{1}^{\prime}, \ldots, w_{m_{1}}^{\prime}$ and $w_{0}^{\prime \prime}, w_{m_{1}+1}^{\prime \prime}, \ldots, w_{M}^{\prime \prime}$ defined for the sets $E_{v}^{\prime}, E_{v}^{\prime \prime}$ by the equations

$$
\left[\begin{array}{c}
w_{0}^{\prime} \\
w_{1}^{\prime} \\
\vdots \\
w_{m_{1}}^{\prime}
\end{array}\right]=\left(\Theta^{\prime}\right)^{-1}\left[\begin{array}{c}
1 \\
0 \\
\vdots \\
0
\end{array}\right], \quad\left[\begin{array}{c}
w_{0}^{\prime \prime} \\
w_{m_{1}+1}^{\prime \prime} \\
\vdots \\
w_{M}^{\prime \prime}
\end{array}\right]=\left[\Theta^{\prime \prime}\right]^{-1}\left[\begin{array}{c}
1 \\
0 \\
\vdots \\
0
\end{array}\right]
$$

satisfy the required conditions, and the polynomials $f_{1}(z), f_{2}(z)$ of respective degrees $n_{1}, n_{2}$ given by (12.1) serve to define $E_{v}^{\prime}, E_{v}^{\prime \prime}$ as PL-domains. After raising $f_{1}(z)$ and $f_{2}(z)$ to appropriate powers, we can assume that $n_{1}=n_{2}$. 
We claim that there exist rational numbers $w_{0}, \alpha_{1}$, and $\alpha_{2}$ with $\alpha_{1}, \alpha_{2}>$ 0 and $\alpha_{1}+\alpha_{2}=1$, such that

$$
\widehat{\mathbf{w}}:=\left[\begin{array}{c}
w_{0} \\
\alpha_{1} w_{1}^{\prime} \\
\vdots \\
\alpha_{1} w_{m_{1}}^{\prime} \\
\alpha_{2} w_{m_{1}+1}^{\prime \prime} \\
\vdots \\
\alpha_{2} w_{M}^{\prime \prime}
\end{array}\right]
$$

satisfies the equation

$$
\left[\begin{array}{l}
1 \\
\overrightarrow{0}
\end{array}\right]=\Theta \widehat{\mathbf{w}}
$$

Since such a solution is unique, the entries in $\widehat{\mathbf{w}}$ must be the numbers $w_{i}$ in (12.6), and our claims that $w_{1}, \ldots, w_{m}$ are positive and $\sum_{i=1}^{m} w_{i}=1$ will be established.

Letting $\widehat{\mathbf{w}}$ be as above, with $w_{0}, \alpha_{1}$ and $\alpha_{2}$ as variables, in view of the block structure of the matrix $\Theta$, and the equations satisfied by the $w_{j}^{\prime}$ and the $w_{j}^{\prime \prime},(12.7)$ reads

$$
\left[\begin{array}{c}
1 \\
\overrightarrow{0}
\end{array}\right]=\left[\begin{array}{c}
\alpha_{1}+\alpha_{2} \\
w_{0}+\alpha_{1}\left(-w_{0}^{\prime}\right)+\alpha_{2} \delta^{\prime} \\
\vdots \\
w_{0}+\alpha_{1}\left(-w_{0}^{\prime}\right)+\alpha_{2} \delta^{\prime} \\
w_{0}+\alpha_{1} \delta^{\prime}+\alpha_{2}\left(-w_{0}^{\prime \prime}\right) \\
\vdots \\
w_{0}+\alpha_{1} \delta^{\prime}+\alpha_{2}\left(-w_{0}^{\prime \prime}\right)
\end{array}\right] .
$$

Consequently, in order to obtain (12.7), we need $w_{0}, \alpha_{1}$ and $\alpha_{2}$ to satisfy

$$
\begin{aligned}
\alpha_{1}+\alpha_{2} & =1, \\
w_{0}-w_{0}^{\prime} \alpha_{1}+\delta^{\prime} \alpha_{2} & =0, \\
w_{0}+\delta^{\prime} \alpha_{1}-w_{0}^{\prime \prime} \alpha_{2} & =0 .
\end{aligned}
$$

Solving, we find

$$
\alpha_{1}=\frac{\delta^{\prime}+w_{0}^{\prime \prime}}{\left(\delta^{\prime}+w_{0}^{\prime}\right)+\left(\delta^{\prime}+w_{0}^{\prime \prime}\right)}, \quad \alpha_{2}=\frac{\delta^{\prime}+w_{0}^{\prime}}{\left(\delta^{\prime}+w_{0}^{\prime}\right)+\left(\delta^{\prime}+w_{0}^{\prime \prime}\right)},
$$




$$
w_{0}=\frac{w_{0}^{\prime} w_{0}^{\prime \prime}-\delta^{\prime} \delta^{\prime}}{\left(\delta^{\prime}+w_{0}^{\prime}\right)+\left(\delta^{\prime}+w_{0}^{\prime \prime}\right)} .
$$

Thus, our assertions hold if $\delta^{\prime}+w_{0}^{\prime}$ and $\delta^{\prime}+w_{0}^{\prime \prime}$ are both positive. However, that is the case, since $-w_{0}^{\prime}=\sum_{j=1}^{m_{1}} \Theta_{i j} w_{j}^{\prime}$ is an average (with positive coefficients) of numbers $\Theta_{i j}$ which are strictly less than $\delta^{\prime}$, and similarly $w_{0}^{\prime \prime}$ is an average of numbers $\Theta_{i j} \leq \delta^{\prime}$, with $\Theta_{i i}<\delta^{\prime}$.

Now let $N$ be a common multiple of the denominators of $\alpha_{1}$ and $\alpha_{2}$, and

$$
F(z)=f_{1}(z)^{N \alpha_{1}} f_{2}(z)^{N \alpha_{2}} .
$$

By the ultrametric inequality and the definition of the equivalence classes for $\sim,\left|f_{1}(z)\right|_{v}$ is constant on $E_{v}^{\prime \prime}$, and similarly $\left|f_{2}(z)\right|_{v}$ is constant on $E_{v}^{\prime}$. Hence $|F(z)|_{v}$ takes the same value, say $R$, at each point of the "boundary" $\partial E_{v}=\bigcup_{i=1}^{M} \partial B\left(a_{i}, r_{i}\right)$. By the construction of $f_{1}(z)$ and $f_{2}(z)$ it follows that

$$
E_{v}=E_{v}^{\prime} \cup E_{v}^{\prime \prime} \subset\left\{z \in \mathbb{P}^{1}\left(\mathbb{C}_{v}\right):|F(z)|_{v} \leq R\right\} .
$$

Let $r^{\prime}$ be such that $\delta^{\prime}=\log _{v}\left(r^{\prime}\right)$. For the same reasons as above, $\left|f_{1}(z)\right|_{v}$ is constant on each "open" ball $B\left(a_{j}, r^{\prime}\right)^{-}$with $a_{j} \neq a_{1}$, and $\left|f_{2}(z)\right|_{v}$ is constant on $B\left(a_{1}, r^{\prime}\right)^{-}$. Suppose now that $x \notin E_{v}$. If $x$ belongs to $B\left(a_{1}, r^{\prime}\right)^{-}$, fix $x_{1} \in \partial B\left(a_{1}, r_{1}\right)$. By the definition of $f_{1}(z)$ and the discussion above,

$$
\left|f_{1}(x)\right|_{v}>\left|f_{1}\left(x_{1}\right)\right|_{v}, \quad \text { while } \quad\left|f_{2}(x)\right|_{v}=\left|f_{2}\left(x_{1}\right)\right|_{v} .
$$

Consequently $|F(x)|_{v}>\left|F\left(x_{1}\right)\right|_{v}=R$. Similarly if $x$ belongs to any other open ball $B\left(a_{j}, r^{\prime}\right)^{-}$then $|F(x)|_{v}>R$. If $x$ belongs to none of these open balls, then $\left|x-a_{i}\right|_{v} \geq r^{\prime}$ for all $i$, and so fixing $x_{1} \in \partial B\left(a_{1}, r_{1}\right)$ we see that $\left|f_{1}(x)\right|_{v}>\left|f_{1}\left(x_{1}\right)\right|_{v}$ and $\left|f_{2}(x)\right|_{v} \geq\left|f_{2}\left(x_{1}\right)\right|_{v}$. Hence again $|F(x)|_{v}>R$. We have thus shown that

$$
E_{v}=\left\{z \in \mathbb{P}^{1}\left(\mathbb{C}_{v}\right):|F(z)|_{v} \leq R\right\} .
$$

By scaling $F(z)$ appropriately we can obtain a polynomial $f(z)$ for which

$$
E_{v}=\left\{z \in \mathbb{P}^{1}\left(\mathbb{C}_{v}\right):|f(z)|_{v} \leq 1\right\}
$$

Since the roots of $f(z)$ have the same relative multiplicities as those of $F(z)$ (namely $w_{1}, \ldots, w_{M}$ ), while

$$
w_{0}=-\sum_{j=1}^{M} \Theta_{i j} w_{j} \quad \text { for any } i,
$$

we have shown that the polynomial defined by (12.1) exhibits $E_{v}$ as a PLdomain. This completes the proof when $\zeta=\infty$.

Finally, suppose $\zeta \neq \infty$. The linear fractional transformation $h(z)=$ $1 /(z-\zeta)$ takes $\zeta$ to $\infty$, and takes a ball $B(a, r)$ not containing $\zeta$ to the ball $B\left(h(a), r /|\zeta-a|_{v}^{2}\right)$. Consequently, $h\left(E_{v}\right)$ is again a finite union of balls. If $g(z)$ is the polynomial constructed by (12.1) for the set $h\left(E_{v}\right)$, with zeros 
supported on the points $h\left(a_{i}\right)$, then $f(z)=g(h(z))$ will be a rational function with poles only at $\zeta$ and zeros supported on the $a_{i}$, such that

$$
E_{v}=\left\{z \in \mathbb{P}^{1}\left(\mathbb{C}_{v}\right):|f(z)|_{v} \leq 1\right\} .
$$

After scaling $f(z)$ by a number $u \in \mathbb{C}_{v}$ with $|u|_{v}=1$, we can assume $f(z)$ has the form (12.4) with $w_{1}, \ldots, w_{M}$ positive. If we now repeat the argument leading to (12.5), it follows that $w_{0}, w_{1}, \ldots, w_{M}$ satisfy

$$
\left[\begin{array}{c}
w_{0} \\
w_{1} \\
\vdots \\
w_{M}
\end{array}\right]=\Theta^{-1}\left[\begin{array}{c}
1 \\
\log _{v}\left(\left|\zeta-a_{1}\right|_{v}\right) \\
\vdots \\
\log _{v}\left(\left|\zeta-a_{M}\right|_{v}\right)
\end{array}\right]
$$

\section{References}

[1] Y. Amice, Les nômbres p-adiques, Presses Universitaires de France, 1975.

[2] M. L. Balinski and H. P. Young, The quota method of apportionment, Amer. Math. Monthly 82 (1975), 701-730.

[3] D. Cantor, On an extension of the definition of transfinite diameter and some applications, J. Reine Angew. Math. 316 (1980), 160-207.

[4] J. W. S. Cassels and A. Fröhlich (eds.), Algebraic Number Theory, Thompson, Washington, 1967.

[5] M. Fekete and G. Szegö, On algebraic equations with integral coefficients whose roots belong to a given set, Math. Z. 63 (1955), 158-172.

[6] E. Hille, Analytic Function Theory, Vol. II, 2nd ed., Chelsea, New York, 1977.

[7] J. Hollstein, Über den transfiniten Durchmesser auf nichtarchimedisch bewerteten Körpern, thesis, University of Heidelberg, 1984.

[8] G. Pólya, Über ganzwertige Polynome in algebraischen Zahlkörpern, J. Reine Angew. Math. 149 (1919), 97-116.

[9] T. Rivlin, Chebyshev Polynomials from Approximation Theory to Algebra and Number Theory, 2nd ed., Wiley, New York, 1990.

[10] R. M. Robinson, Conjugate algebraic integers in real point sets, Math. Z. 84 (1964), 415-427.

[11] R. Rumely, Capacity Theory on Algebraic Curves, Lecture Notes in Math. 1378, Springer, New York, 1989.

[12] —, The Fekete-Szegö theorem with splitting conditions: Part I, Acta Arith. 93 (2000), 99-116.

[13] M. Tsuji, Potential Theory in Modern Function Theory, Maruzen, Tokyo, 1959 (reprinted: Chelsea, New York, 1975).

Department of Mathematics

University of Georgia

Athens, GA 30602, U.S.A.

E-mail: rr@alpha.math.uga.edu 